\begin{abstract}
Title of Document:

AN INTEGRAL MODEL FOR TURBULENT FLAME RADIAL LENGTHS UNDER A CEILING

Haiwen Ding, Master of Science, 2010

Directed By:

Professor, James G. Quintiere, Department of Fire Protection Engineering

An analytical study using an integral model for turbulent flame radial lengths under a ceiling is presented. Dimensionless equations give results in terms of $Q^{*}$-dimensionless firepower, and $\mathrm{D} / \mathrm{H}$-- the ratio of fire diameter to ceiling height. The model used an empirical relationship for the mixing ratio of air entrained to stoichiometric air needed for the ceiling jet flame. This value varies from 9.6 for short radial flames to 1 for long flames that tend to become laminar. Predictions from the model are in good agreement with the experimental data from previous work found in the literature. They span a range that contains data where the fire plume flame just touches the ceiling to the case where long ceiling flames become laminar. An alternative empirical fit of the theoretical results finds that the radial flame length is independent of ceiling height once the flame hits the ceiling.
\end{abstract}




\title{
AN INTEGRAL MODEL FOR TURBULENT FLAME RADIAL LENGTHS UNDER A CEILING
}

\author{
By \\ Haiwen Ding \\ Thesis submitted to the Faculty of the Graduate School of the \\ University of Maryland, College Park, in partial fulfillment \\ of the requirements for the degree of \\ Master of Science \\ 2010
}

Advisory Committee:

Professor James G. Quintiere, Chair

Associate Professor André W. Marshall

Assistant Professor Peter B. Sunderland 
C Copyright by Haiwen Ding 


\section{Acknowledgements}

This study was funded by Professor John L. Bryan, the Hartford Steam Boiler Inspection and Insurance Company, and the Cerberus Technologies, Inc.

I want to express my sincere gratitude to Dr. James G. Quintiere for guiding me throughout this study, and for his knowledge, wisdom, passion, patience and tolerance that enabled me to accomplish this current work and will continue to influence me in my life.

I am indebted to Ning Ren and Lei Wang for their invaluable advice in solving some technical problems in this work.

I am grateful to my parents for introducing me to this field and for their constant support on my way of pursuing my Master's degree.

I want to give warm thanks to all my friends for their encouragement, especially Praveen Narayanan and Blake Riddick.

I would like to thank the rest of my committee members, Dr. André W. Marshall and Dr. Peter B. Sunderland, for their efforts in my thesis defense. 


\section{Table of Contents}

Acknowledgements ..........................................................................................ii

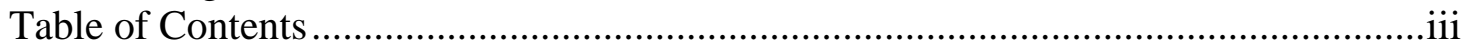

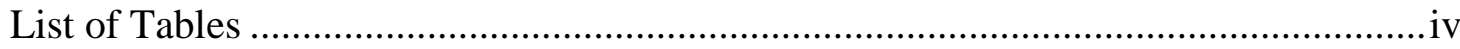

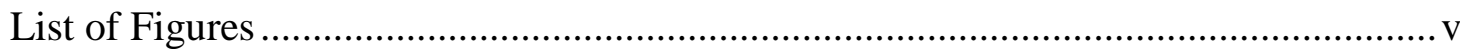

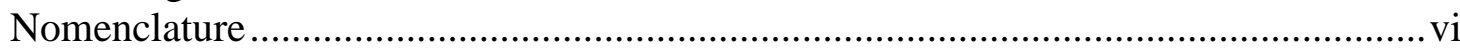

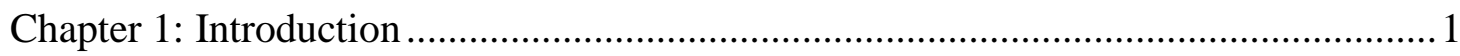

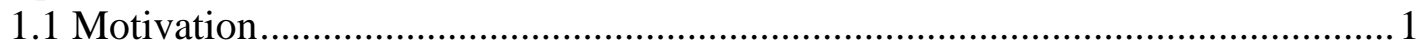

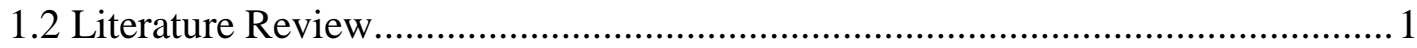

1.3 Research Objectives .................................................................................. 2

Chapter 2: Approach ......................................................................................... 4

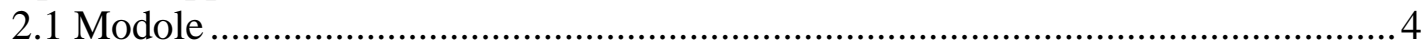

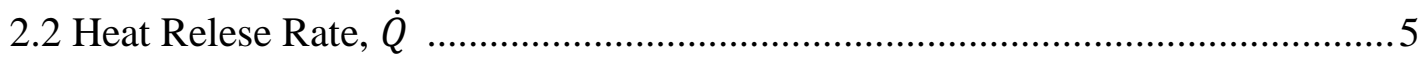

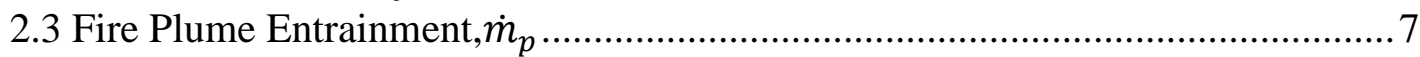

2.4 Fire Plume at the Turning Region.............................................................. 9

2.5 Initial Conditions for the Ceiling Jet ............................................................ 9

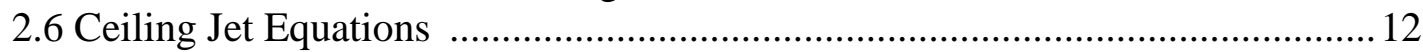

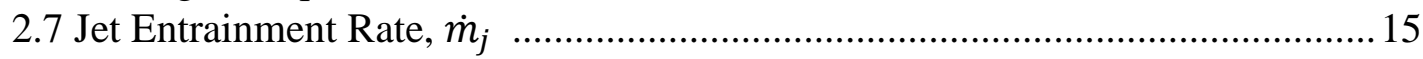

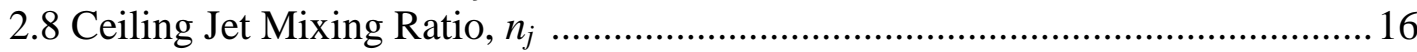

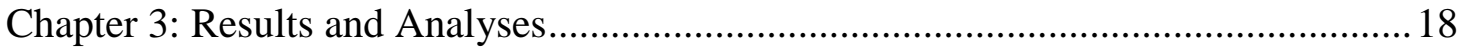

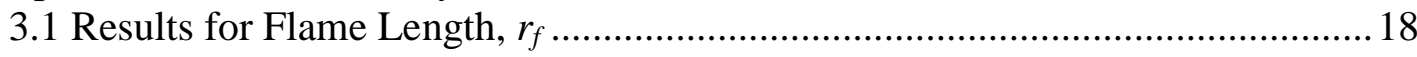

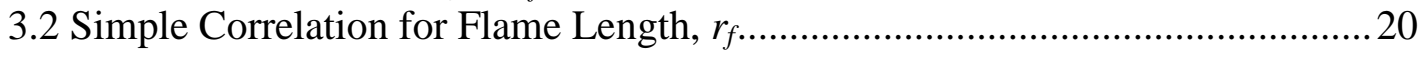

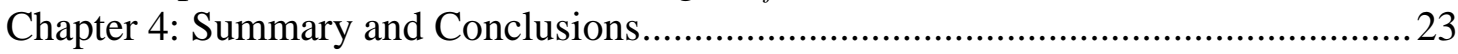

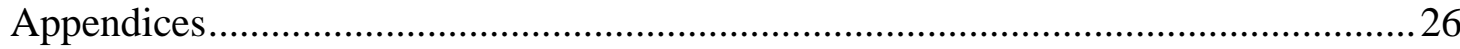

Appendix A: Data and Calculations for Ceiling Jet Mixing Ratio, $n_{j} \ldots \ldots \ldots \ldots \ldots \ldots . . . . .26$

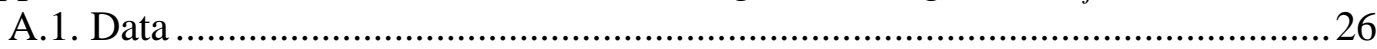

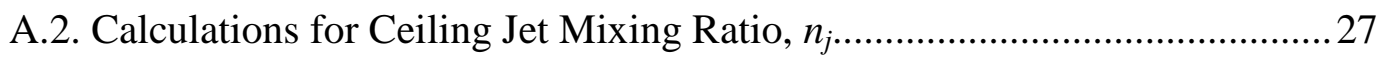

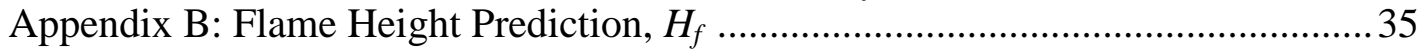

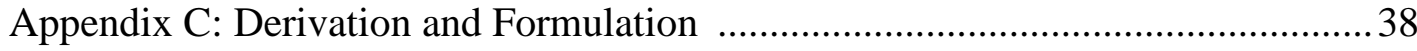

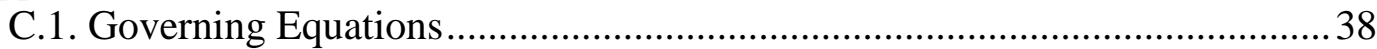

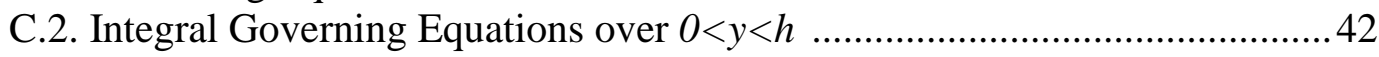

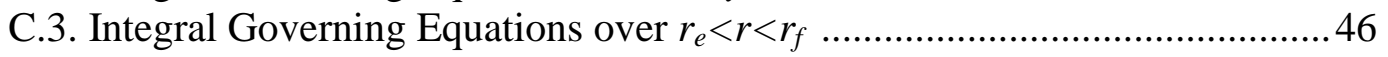

Appendix D: Calculations for Flame Length, $r_{f}$, and Error Examination................. 48

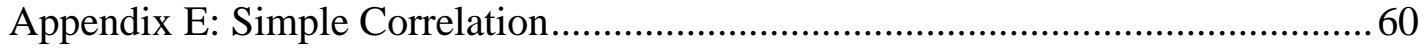




\section{List of Tables}

Table 1. Range of available ceiling flame length data........................................ 2

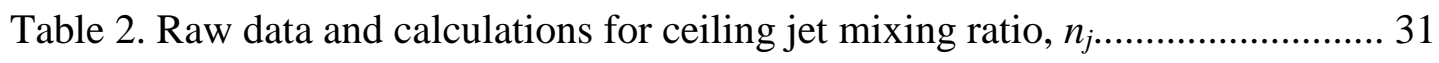

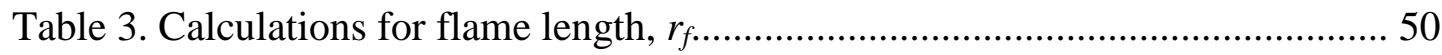

Table 4. Direct comparison of theoretical prediction vs. data for flame length, $r_{f \ldots . . .58}$ 


\section{List of Figures}

Figure 1. Correlation of radial to free flame length data................................. 1

Figure 2. Schematic of the model......................................................... 6

Figure 3. Variation of ceiling jet mixing with Richardson number at the flame tip.17

Figure 4. Theoretical results compared to data for radial flame length...................18

Figure 5. Direct comparison of predicted and experimental results......................19

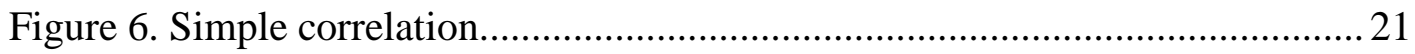

Figure 7. Illustration showing $r_{f}$ is independent of $H$ for given $\dot{Q}$ and $D \ldots \ldots \ldots \ldots . . . .21$

Figure 8. Radial flame length in terms of free flame height.............................. 22

Figure 9. Free flame height and radial flame length.......................................... 23

Figure 10. Free flame height prediction tailored to data of You.......................... 36

Figure 11. Free flame height predictions compared to a wide range of data........... 37

Figure 12. Correlations of theoretical results for radial flame length..................... 61 


\section{Nomenclature}

Latin symbols

\begin{tabular}{|c|c|}
\hline$C_{i}$ & generic constants used in equations (unitless) \\
\hline$D$ & fire source diameter $(\mathrm{m})$ \\
\hline$E$ & entrainment coefficient (unitless) \\
\hline$f$ & friction factor (unitless) \\
\hline$g$ & gravity $\left(9.8 \mathrm{~m} / \mathrm{s}^{2}\right)$ \\
\hline$h$ & ceiling jet flame thickness $(\mathrm{m})$ \\
\hline$H$ & distance between the fire source and the ceiling $(\mathrm{m})$ \\
\hline$m$ & mass $(\mathrm{kg})$ \\
\hline$n$ & ratio of the entrained air to stoichiometric air (unitless) \\
\hline$q$ & energy generated $(\mathrm{kJ})$ \\
\hline$r$ & radial length $(\mathrm{m})$ \\
\hline $\operatorname{Re}$ & Reynolds number (unitless) \\
\hline $\mathrm{Ri}$ & Richardson number (unitless) \\
\hline$S$ & stoichiometric air/fuel ratio (unitless) \\
\hline$T$ & temperature $\left(T_{\infty}=298 \mathrm{~K}\right)$ \\
\hline$u$ & vertical velocity in the ceiling jet $(\mathrm{m} / \mathrm{s})$ \\
\hline$v$ & horizontal velocity in the ceiling jet $(\mathrm{m} / \mathrm{s})$ \\
\hline$w$ & vertical velocity in the plume $(\mathrm{m} / \mathrm{s})$ \\
\hline$\chi_{r}$ & radiation fraction (unitless) \\
\hline$X_{T}$ & energy loss fraction (unitless) \\
\hline
\end{tabular}




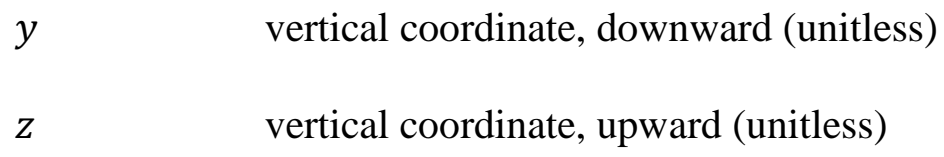

Greek and other symbols

$\theta$

$\lambda$

$\mu \quad$ dynamic viscosity $(\mathrm{kg} / \mathrm{m}-\mathrm{s})$

$\rho \quad$ density $\left(\rho_{\infty}=1.2 \mathrm{~kg} / \mathrm{m}^{3}\right)$

$\varphi \quad$ entrainment parameter (unitless)

$\Delta h_{c} / s \quad$ heat of combustion per unit mass of air $(2.97 \mathrm{~kJ} / \mathrm{g})$

$\begin{array}{ll}\begin{array}{ll}\text { Subscripts } \\ e\end{array} & \text { air } \\ f & \text { starting point of the ceiling jet } \\ i & \text { end point of the ceiling jet } \\ j & \text { ceiling jet } \\ p & \text { plume } \\ \infty & \text { ambient conditions }\end{array}$

Superscripts
$\left({ }^{\circ}\right)$
( ) per unit time
()$^{\prime \prime \prime}$
( ) per unit volume 
()$^{*}$ dimensionless form of ()

()$^{\prime} \quad$ dimensionless form of $($ ) 


\section{Chapter 1: Introduction}

\section{$\underline{1.1 \text { Motivation }}$}

The ability to predict the length of a flame under a ceiling is important in assessing fire hazard. The ceiling heat flux will increase dramatically in the flame region, and the potential for flame spread on a combustible ceiling is now possible. Despite the importance of this problem, relatively little work has been done to address it. In contrast, much data has been taken for free axi-symmetric vertical turbulent flames, and consensus correlations exist to predict the turbulent flame length [e.g., 1].

\subsection{Literature Review}

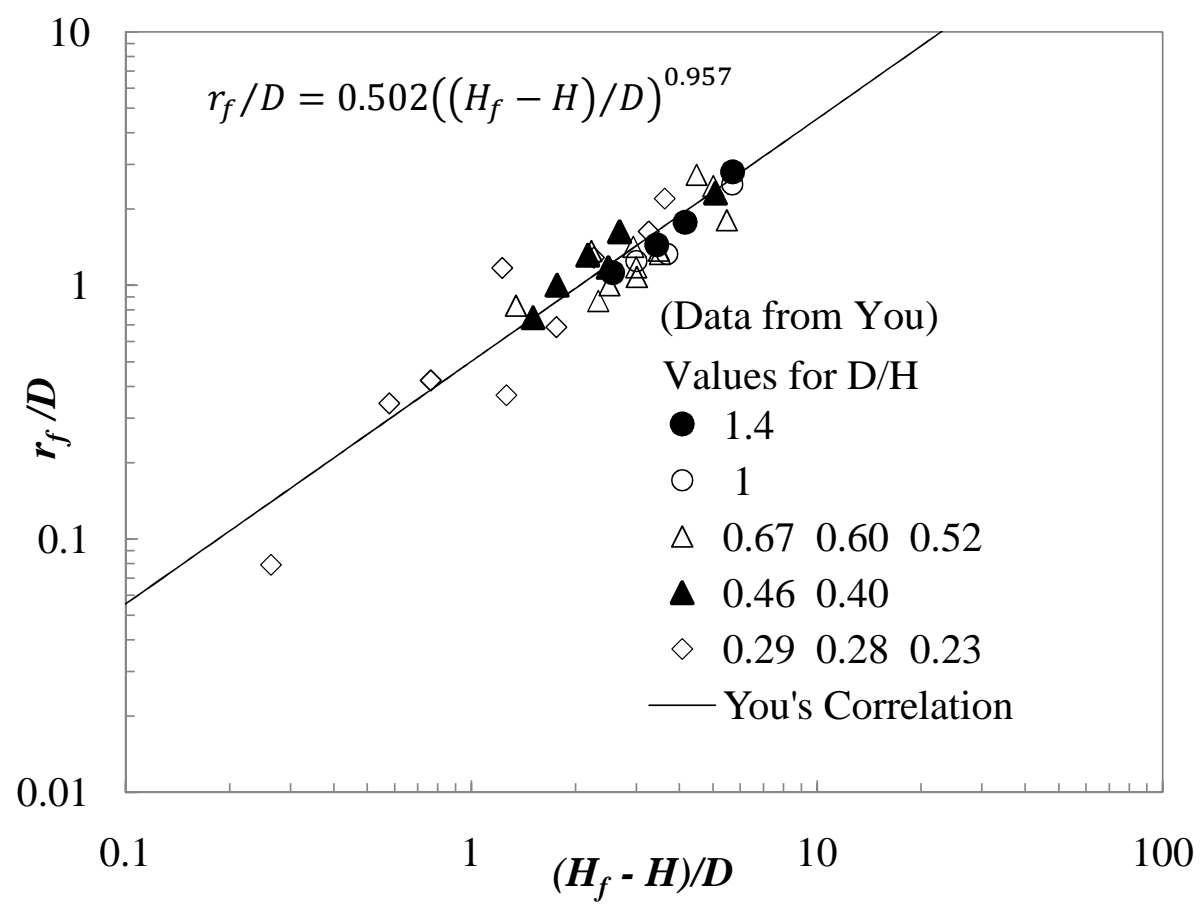

Figure 1. Correlation of radial to free flame length data [2]. 
The only available correlation to predict the flame extension under a ceiling was given by You and Faeth [2]. They based their correlation on small-scale data. Babrauskas [3] reviewed the problem, and attempted to develop a predictive method for the ceiling flame. He assumed similar entrainment rates into the free plume and the ceiling jet, but no analytic equation emerged from his analysis. Gross [4] later obtained data for larger fire scales, and Kokkala and Rinkenin [5] reported further small-scale data. The latter [5] data had curious laminar characteristics as the flames exhibited various systematic shapes, such as a "laminar disc", "circular rings", and "a flower pattern". The research of You [7-8] took the study of ceiling jet flames further, but no new correlation resulted. Recently, Heskestad and Hamada [9] studied the "strong" fire plume interaction with a ceiling, and some limited ceiling flame data can be extracted from their work. The ensemble of available data for the ceiling jet flame is described in Table 1. The detailed use of data is described in Appendix A.

Table 1. Range of available ceiling flame length data.

\begin{tabular}{ccccc}
\hline Source & $\dot{Q}(\mathrm{~kW})$ & $D(\mathrm{~cm})$ & $H(\mathrm{~cm})$ & $r_{f}(\mathrm{~cm})$ \\
\hline You \& Faeth [2] & $0.36-7.89$ & $3.5-10.7$ & $7.6-33.6$ & $0.6-32$ \\
\hline Kokkala \& Rinkinen [5] & $4.03-11.04$ & 6 & $2-10$ & $12-29$ \\
\hline Gross [4] & $104-283$ & 61 & 50 & $70-110$ \\
\hline Heskestad \& Hamada [9] & $23.2-764$ & $15-61$ & $56-151$ & $12-191$ \\
\hline
\end{tabular}

\section{$\underline{1.3 \text { Research Objectives }}$}

Related studies for the non-combusting ceiling jet give valuable information. Alpert [6] first presented on integral model for the ceiling jet. Aspects of his approach will be followed in the present analysis. Yao and Marshall [11] present valuable analog data for saltwater plumes 
and their interaction with the ceiling, especially giving insight into the dynamics of the turning region.

We seek to use a simple integral model for the flame region in the ceiling jet, and attempt to obtain approximate solutions that will help to generalize the radial flame length data. Also we seek to examine if the correlation given by You and Faeth [2] will be supported by such an analysis. Their correlation, with their data, is shown in Figure 1. While the correlation as shown is generally good, to use this correlation, a prediction for the free flame height is needed. Moreover, it must be seen if the correlation can predict the entire data ensemble of Table 1 . We seek to see if an approximate integral model can give more general results. 


\section{Chapter 2: Approach}

\section{$\underline{2.1 \text { Model }}$}

The model is based on the approach that the fuel supply rate is exhausted when all of the reacting air is entrained. This end position gives the radial flame extent. The entrainment rate for the vertical fire plume and the ceiling jet must be known. For the free vertical plume, the results of Grove and Quintiere [1] give an expression for that entrainment rate. No result exists for the entrainment rate into the ceiling flame. However, the integral framework of the successful Alpert [6] non-combustion ceiling jet can be used to obtain the needed entrainment rate. In addition, the mixing rate for combustion $-n$, the ratio of the entrained air to stoichiometric air - is needed for both the plume and the jet. For the plume, it was found that $n$ is 9.6 [1]. That is, about 10 times stoichiometric air must be entrained to complete combustion. The value for $n$ in the ceiling jet is not known. Moreover, it is not likely to be a constant as in the free fire plume; especially since the ceiling jet can re-laminarize.

Boundary and initial conditions are also needed. The plume will give the initial condition. The ceiling boundary condition for heat transfer is problematic. Alpert [6] found that the ceiling heat transfer had surprisingly small influence on his results in the ceiling jet region. However, correlations for the free plume and the same plume with a ceiling give dramatically different temperatures [14]. This difference indicates that the greatest loss of heat from the flame is in the stagnation region of the impinging zone. Therefore, we will make some simplifying approximations here. It will be considered a constant fraction of the available plume energy; 
after the turning region, an adiabatic assumption is made for the horizontal ceiling jet consistent with the findings of Alpert [6].

The dynamics of the ceiling jet will be computed using an integral model with uniform velocity and temperature in the normal direction. These variables can then be computed along with the jet thickness as a function of the radius, $r$. As a result of these considerations and assumptions, the jet entrainment rate can be formulated, and in turn the mixing rate for the jet will be estimated. The model formulation is described for each component below.

\subsection{Heat Release Rate, $\dot{Q}$}

The total combustion energy from the fuel source is consumed in three regions: (1) the vertical fire plume region, (2) the turning region, and (3) the horizontal ceiling jet flame region after the turning region. These regions are displayed in Figure 2 along with some nomenclature for the model. The rates of air entrainment into the plume and jet regions determine the rate of combustion. 


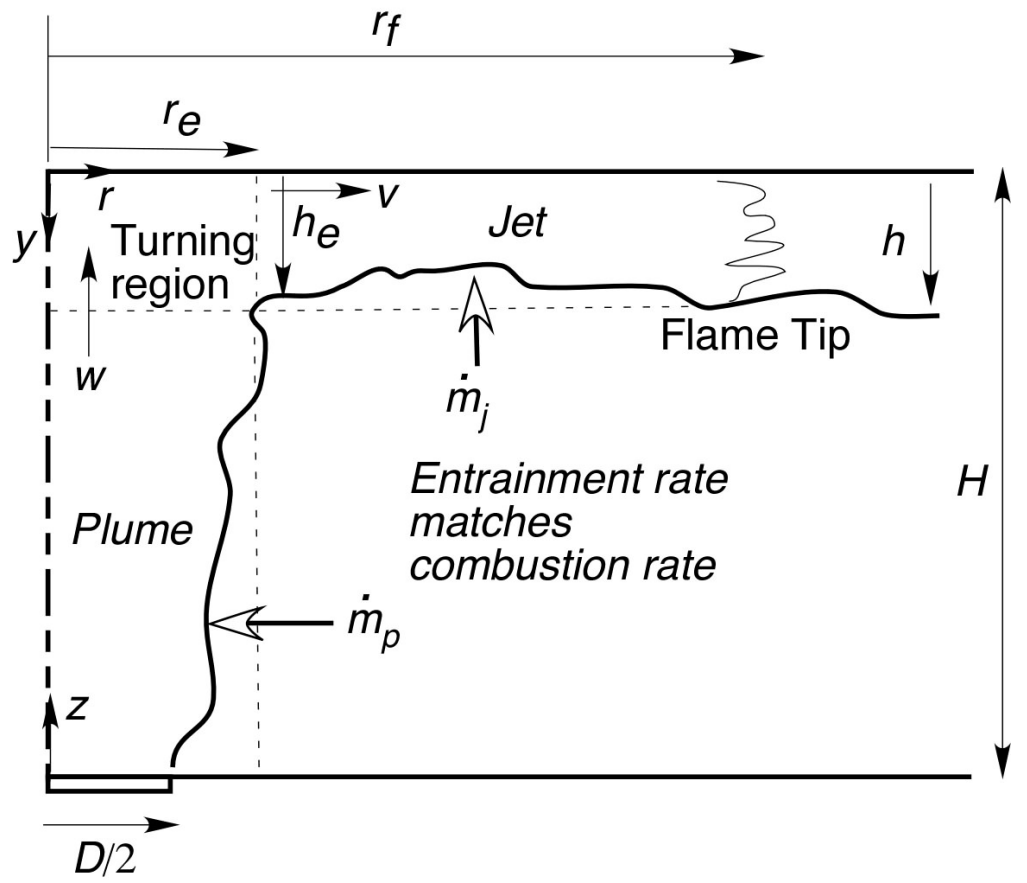

Figure 2. Schematic of the model.

At the tip of the flame, no more fuel remains. Therefore the fuel mass release rate is equal to the fuel reaction rate.

$\dot{m}_{F, \text { sup }}=\dot{m}_{F, \text { react }}$

Replace the fuel reaction rate in terms of air reaction rate. From the stoichiometry,

$1(\mathrm{~g})$ fuel $+s(\mathrm{~g})$ air $=(1+s)(\mathrm{g})$ product.

The stoichiometric air to fuel mass ratio is given as $s$. Then rate of fuel reacted is related to the air as

$\dot{m}_{F, \text { react }}=\dot{m}_{A, \text { react }} / s$.

The rate of air entrained into the fire plume and the ceiling jet is in excess of the air needed for combustion. Actually it is found that about 10 times more than stoichiometric air is entrained 
over the flame length. This appears to be a turbulent-combustion property at the plume and may not depend on fuel. In terms of the mixing ratio for each region, the rate of air entrainment is

$\dot{m}_{i}=n_{i} \dot{m}_{A, \text { react }}$.

where the subscript- $i$ can refer to the plume $(p)$ or the ceiling jet $(j)$. Take the air entrainment rate in the vertical fire plume region as $\dot{m}_{p}$. This is $n_{p}$ times the stoichiometric air entrainment rate in the plume. Similarly, the air entrainment rate in the horizontal ceiling jet region $\dot{m}_{j}$ is $n_{j}$ times the stoichiometric air in the jet. Then

$\dot{m}_{F, \text { sup }}=\left(\frac{\dot{m}_{p}}{n_{p}}+\frac{\dot{m}_{j}}{n_{j}}\right) \frac{1}{s}$.

Multiply Eq. (5) by the heat of combustion $\Delta h_{c}$. Note that $\dot{m}_{F, \text { sup }} \Delta h_{c}$ is the heat release rate $\dot{Q}$.

$\dot{Q}=\left(\frac{\dot{m}_{p}}{n_{p}}+\frac{\dot{m}_{j}}{n_{j}}\right)\left(\frac{\Delta h_{c}}{s}\right)$

This expression will be used to obtain the flame length. It should be noted that $\Delta h_{c} / s$ is nearly a constant for most hydrocarbon fuels, $\sim 2.97 \mathrm{~kJ} / \mathrm{g}$-air. Formulation of the entrainments rates will complete this expression and allow for a solution of the entrainment (or flame) length in the radial direction. The value of $n_{p}$ for the fire plume is taken 9.6 [1], and the value of $n_{j}$ for the ceiling jet needs to be determined.

\subsection{Fire Plume Entrainment Rate, $\dot{m}_{p}$}

Grove and Quintiere [1] gave the formula of entrainment rate in the vertical fire plume region as a function of flame elevation, $z$,

$\dot{m}_{p}(z)=\rho_{\infty} g^{1 / 2} D^{5 / 2} C_{e}(z / D)^{1 / 2}\left(1+2 C_{l} z / D\right)^{2}$ 
where

$z$ height above the fire source,

$D$ fire source diameter,

$C_{l}$ fire plume radius constant, $C_{l}=0.179$,

$C_{e}$ entrainment coefficient, adjusted to fit the flame length data of You [2],

$C_{e}=0.026\left[\lambda\left(1-\chi_{r}\right)\right]^{1 / 2}$, where

$\lambda \equiv \Delta h_{c} / s C_{p} T_{\infty} \approx 9.97$

$\chi_{r}$ radiation fraction.

Several fuel sources were used in You's experiments, including methanol, ethanol, propanol and pentane. $\chi_{r}$ is found to be 0.15 and 0.24 [13], for methanol and ethanol, respectively, and we found a reasonable approximation of $\chi_{r}$ for all fuels to be $\chi_{r}=0.20$.

The formulation of Eq. (7) has been found to have good accuracy with flame entrainment data (+/-20\%), and accounts for the flame's base diameter. The constant 0.026 was selected to match the data of You [2] for his free plumes to maintain consistency in comparing with his ceiling flame data (see Appendix B). The value of Grove and Quintiere [1] is about twice.

The value of $z$ is taken at the elevation where the plume intersects the ceiling jet, $H$-he, as shown in Figure 2, where

$H$ distance between the fire source and the ceiling,

$h_{e}$ flame thickness at the starting point of the ceiling jet.

Information about the turning region will allow the plume to be connected to the ceiling jet. 


\subsection{Fire Plume at the Turning Region}

In order to begin the solution for the ceiling jet, values for the fire plume variables are needed at the turning region. These are taken from the correlations of Grove and Quintiere [1]. Their solution in the flame zone is given as:

Plume radius

$$
r_{p}=D / 2+C_{l} z
$$

Centerline velocity

$w_{p}=C_{v}(g z)^{1 / 2}, C_{v}=0.720\left(\lambda\left(1-\chi_{r}\right)\right)^{1 / 2}$,

Centerline temperature

$\theta_{p} \equiv\left(T-T_{\infty}\right) / T_{\infty}=0.347 \lambda\left(1-\chi_{r}\right)$

\subsection{Initial Conditions for the Ceiling Jet}

Conservation laws applied to the turning region will give the initial conditions for the ceiling jet at $r=r_{e}$. As the ceiling jet thickness at the start is roughly about $0.1 H$ [10], the plume starting values will be taken at $z=H$ as an approximation. Also the profiles in the plume and the ceiling jet, normal to the flow direction, are approximated as uniform. We do not expect these approximations to have a significant impact on the ultimate result for radial flame length. The following initial conditions are developed.

Radius:

$r_{e}=r_{p}(H)$ 
Alpert [6] assumed the horizontal flow velocity at the starting point of the ceiling jet is equal to the vertical velocity at the ending point of the fire plume. This might be justified by an inviscid flow for the turn. Measurements by Yao and Marshall [11] confirm that this is a reasonable assumption. Therefore,

Velocity:

$v_{e}=w_{p}(H)$

The flame temperature at the starting point of the ceiling jet is lower than the flame temperature at the end point of the fire plume, as significant heat transfer occurs in the stagnation region of the ceiling. This heat transfer depends on the flow and the ceiling material. Heskestad's correlations [14] for the free plume and the same plume with a ceiling give dramatically different temperatures.

The centerline mean excess temperature at $z$ above the virtual origin $z_{0}$ is given as

$T-T_{\infty}=9.1\left(T_{\infty} /\left(g c_{P}^{2} \rho_{\infty}^{2}\right)\right)^{1 / 3} \dot{Q}^{2 / 3}\left(z-z_{0}\right)^{-5 / 3}$

or equivalently, at ceiling height $H$,

$\left(T-T_{\infty}\right) / T_{\infty}=9.1 Q_{H}^{* 2 / 3}, Q_{H}^{*}=\dot{Q} /\left(\rho_{\infty} c_{p} T_{\infty} g^{1 / 2} H^{5 / 2}\right)$.

In the ceiling jet, $r / H \leq 0.2$, the correlation for the temperature is given as

$\left(T-T_{\infty}\right) / T_{\infty}=6.3 Q_{H}^{* 2 / 3}$.

As an approximation, it is represented as a heat loss fraction of the initial flow energy, and related to the starting temperature of the jet as $X_{T}=\left(T_{p}-T_{e}\right) /\left(T_{p}-T_{\infty}\right)$. 
$T_{p}-T_{e}$ represents the heat loss to the ceiling, and $T_{p}-T_{\infty}$ represents the energy in the fire plume. From Eqns. $(14 \& 15)$, we observe a $30 \%$ heat loss to the ceiling (i.e. $X_{T}=(9.1-6.3) / 9.1=$ $0.3)$. Thus.$X_{T}$ is estimated to be 0.3 as a representative value based on current correlations. The same value is also found from analyzing the measurements of You [10]. Then, it follows that $\theta_{e}=\left(1-X_{T}\right) \theta_{P}$ where $\theta_{i} \equiv\left(T_{i}-T_{\infty}\right) / T_{\infty}$.

The variables in the ceiling jet formulation will consist of velocity, temperature, and jet thickness, $h$. An initial value, $h_{e}$, is needed for $h$. By considering a control volume encompassing the turning region in Figure 2, the flame thickness at the starting point of the ceiling jet is obtained by performing a mass conservation of the turning region. Uniform, top-hat profiles are assumed for all velocity and temperatures. The densities are directly related to temperatures through the perfect gas law at constant pressure.

The mass flow going into the turning region at the end point of the vertical fire plume is

$\dot{m}_{i n}=\rho_{p} \pi r_{e}^{2} w_{p}$

where

$\rho_{p}$ flow density in the vertical fire plume region.

The mass flow coming out of the turning region at the starting point of the horizontal ceiling jet is

$\dot{m}_{\text {out }}=\rho_{e} 2 \pi r_{e} h_{e} v_{e}$

where

$\rho_{e}$ flow density in the turning region.

Conservation of mass in the turning region gives 
$h_{e}=r_{e} \rho_{p} / 2 \rho_{e}$.

Substitution of the ideal gas law,

$\rho_{p} T_{p}=\rho_{e} T_{e}$,

and substitution of $X_{T}$ give

$h_{e}=\left(r_{e} / 2\right)\left[1-X_{T}+X_{T} T_{\infty} / T_{p}\right]$

where $X_{T} T_{\infty} / T_{p}$ can be neglected since its typical value is of the order of 0.01 .

Thickness:

$h_{e}=r_{e}\left(1-X_{T}\right) / 2$

Thus, Eqns. $(11,12,17 \& 23)$ give the initial conditions for the ceiling jet region. They are now used to solve the governing equations for the ceiling jet.

\subsection{Ceiling Jet Equations}

The ceiling jet equations are derived as in the boundary layer character where $h / H$ is small (of the order of 0.1), and the Reynolds $(\rho v h / \mu)$ number is large, $1 / \operatorname{Re} \sim\left(h_{e} / H\right)^{2}$. We make the following assumptions for these equations:

1. Bousinnesq approximation in which density is constant, except in the buoyancy term.

2. Adiabatic in the ceiling jet, i.e. no heat loss to the ceiling.

3. Pressure variation across the jet is approximately constant in the flame.

4. Combustion rate is controlled by air entrainment.

The pressure assumption eliminates the buoyancy term in the radial momentum equation retained by others $[6,7,10]$. This omission can be shown to be small. We believe these assumptions will 
have no serious effect on the entrainment rate that is being sought to complete the solution for the radial flame length. Detailed derivation for ceiling jet equations are shown in Appendix C. With the preceding assumptions, the governing equations are given as

Mass:

$\frac{1}{r} \frac{\partial(r v)}{\partial r}+\frac{\partial u}{\partial y}=0$

Momentum:

$\rho_{\infty}\left(v \frac{\partial v}{\partial r}+u \frac{\partial v}{\partial y}\right)=\mu \frac{\partial^{2} v}{\partial y^{2}}$

Energy:

$\rho_{\infty} c_{p}\left[v \frac{\partial\left(T-T_{\infty}\right)}{\partial r}+u \frac{\partial\left(T-T_{\infty}\right)}{\partial y}\right]=k \frac{\partial^{2}\left(T-T_{\infty}\right)}{\partial y^{2}}+\dot{q}^{\prime \prime \prime}$

Integration of the governing equations over y is carried out following Alpert [6] and You [7, 8] using top-hat profiles, i.e. uniform quantities along the $y$ direction. The ceiling is considered to be adiabatic, and not other heat loss occurs in the flame. The following ordinary differential equations result for the axisymmetric ceiling jet flame:

Mass:

$\frac{d}{d r}(r v h)=E r v$

Momentum:

$\frac{d}{d r}\left(r v^{2} h\right)=-f r v^{2} / 2$

Energy:

$\frac{d}{d r}(r v h \theta)=\operatorname{Erv} \lambda / n_{j}$ 
where $E$ is the entrainment coefficient, and $u_{\text {ent }}=E v$ represents the entrainment velocity for the turbulent model. $f$ is the friction factor. In general, the entrainment coefficient depends on the local Richardson number $\left(\mathrm{Ri}=g \theta h / v^{2}\right)$, and the friction factor depends on the Reynolds number $(\operatorname{Re}=\rho v h / \mu)$.

Alpert [6] adopted the relationship to describe the entrainment coefficient as

$$
E=E_{j} e^{-\alpha R i}
$$

where the fully turbulent jet entrainment coefficient is $E j=0.075$ for small density changes, and $\alpha \approx 5$. By adjusting $E j$ for large density effects, we can write [15]

$$
E_{j}=0.075\left(\rho / \rho_{\infty}\right)^{1 / 2} \text {. }
$$

Now we wish to evaluate $E$ as a constant to simplify the integration of the equations. We chose to evaluate $E$ at the flame tip

$E_{f}=\left(0.075 /\left(\theta_{f}+1\right)^{1 / 2}\right) e^{-5 \mathrm{Ri}_{f}}$

where the corresponding Richardson number is evaluated at the flame tip.

He took the friction factor as a constant ranging from 0.002 to 0.02 . As the effect of the friction factor is small compared to that of the entrainment coefficient, $f$ was taken as 0.02 in the subsequent computations.

The governing integral equations are integrated for constant $f$ and $E$ from the starting position to the flame tip. Note that the mixing ratio for the ceiling jet, $n j$, has not been specified. We would like to specify it as a constant, and will in the integration, but at this point have no idea what value to assign. The results follow:

$h_{f}=(E+f / 4) r_{f}-\left(r_{e} / r_{f}\right)\left[(E+f / 4) r_{e}-h_{e}\right]$ 


$$
\begin{aligned}
& v_{f}=C_{v}(g H)^{1 / 2}\left[(E+f / 4)\left(r_{e}{ }^{2} / r_{f} h_{f}-r_{f} / h_{f}\right)+1\right]^{(E+f / 2) /(2 E+f / 2)} \\
& \theta_{f}=\left(\left(r_{f} v_{f} h_{f}-r_{e} v_{e} h_{e}\right)\left(\lambda / n_{j}\right)+r_{e} v_{e} h_{e} \theta_{e}\right) /\left(r_{f} v_{f} h_{f}\right)
\end{aligned}
$$

\subsection{Jet Entrainment Rate, $\dot{m}_{j}$}

To complete the solution for $r_{f}$, the radial flame length, the entrainment rate into the ceiling jet up to the flame tip needs to be computed. By conservation of mass, it follows that

$d \dot{m}_{j}=\rho_{\infty} u_{e n t} 2 \pi d r$

with $u_{e n t}=E v$.

Integration from the onset of the ceiling jet flame, $r_{e}$, to the flame tip, $r_{f}$, yields the formula for the air entrainment in the ceiling jet.

$\dot{m}_{j}=2 \pi \rho_{\infty}\left((r v h)_{f}-(r v h)_{e}\right)$

Closure of the problem involves substituting Eqns. (7, 33, \& 36) in Eq. (6) to give a relationship between the overall energy release rate and the radial flame length.

$\dot{Q}=\left(\rho_{\infty} \Delta h_{c} / s\right)\left[g^{1 / 2} D^{5 / 2} C_{e}(z / D)^{1 / 2}\left(1+2 C_{l} z / D\right)^{2} / 9.6+2 \pi\left(r_{f} v_{f} h_{f}-r_{e} v_{e} h_{e}\right) / n_{j}\right]$

$z=H-h_{e}$

However, the mixing ratio for the ceiling jet, $n j$, is still unknown. Here we resort to an empirical fix. This fix is justified, as in any approximate solution it is expected that to satisfy the data an empirical constant is usually needed. That empiricism is expressed through the value of $n j$. 


\subsection{Ceiling Jet Mixing Ratio, nj}

The first three data sets illustrated in Table 1 were used to investigate how $n j$ might vary based on using the data to satisfy Eq. (37). Full results are shown in Appendix A. This variation ranged from about 1 to 10 . It suggested that $n j$ would represent mixing into a laminar flame as stoichiometric conditions would apply for $n j=1$, and to representing the turbulent mixing of the plume for $n j=9.6$. The Richardson number is the parameter that controls the nature of the turbulent mixing, so $n j$ was investigated as a function of $\mathrm{Ri}_{f}$. Figure 3 shows these results. It is seen in the figure that there is also an effect of $\mathrm{D} / \mathrm{H}$. As the flame base diameter becomes larger, the vertical flame becomes more laminar-like near its base - small $H$. It is generally expected that as the $\mathrm{Ri}$ increases to a value of about 0.3 , turbulent mixing is suppressed and laminarization of the jet can occur [12]. While these data do scatter, there is a definite trend that shows $n j$ decreases to values near 1 , and a family of curves pertains to $D / H$. The functionality of the dependence of $n j$ on $\mathrm{Ri}_{f}$ and $D / H$ is somewhat arbitrary, and the following expression was selected as a reasonable fit that follows the data.

$n_{j}=9.6 \exp \left[-\left(2.26\left(R i_{f}-R i_{e}+0.05\right) /(0.197-0.091 D / H)\right)\right]$

The smooth curves in Figure 3 indicate the empirical correlation. 


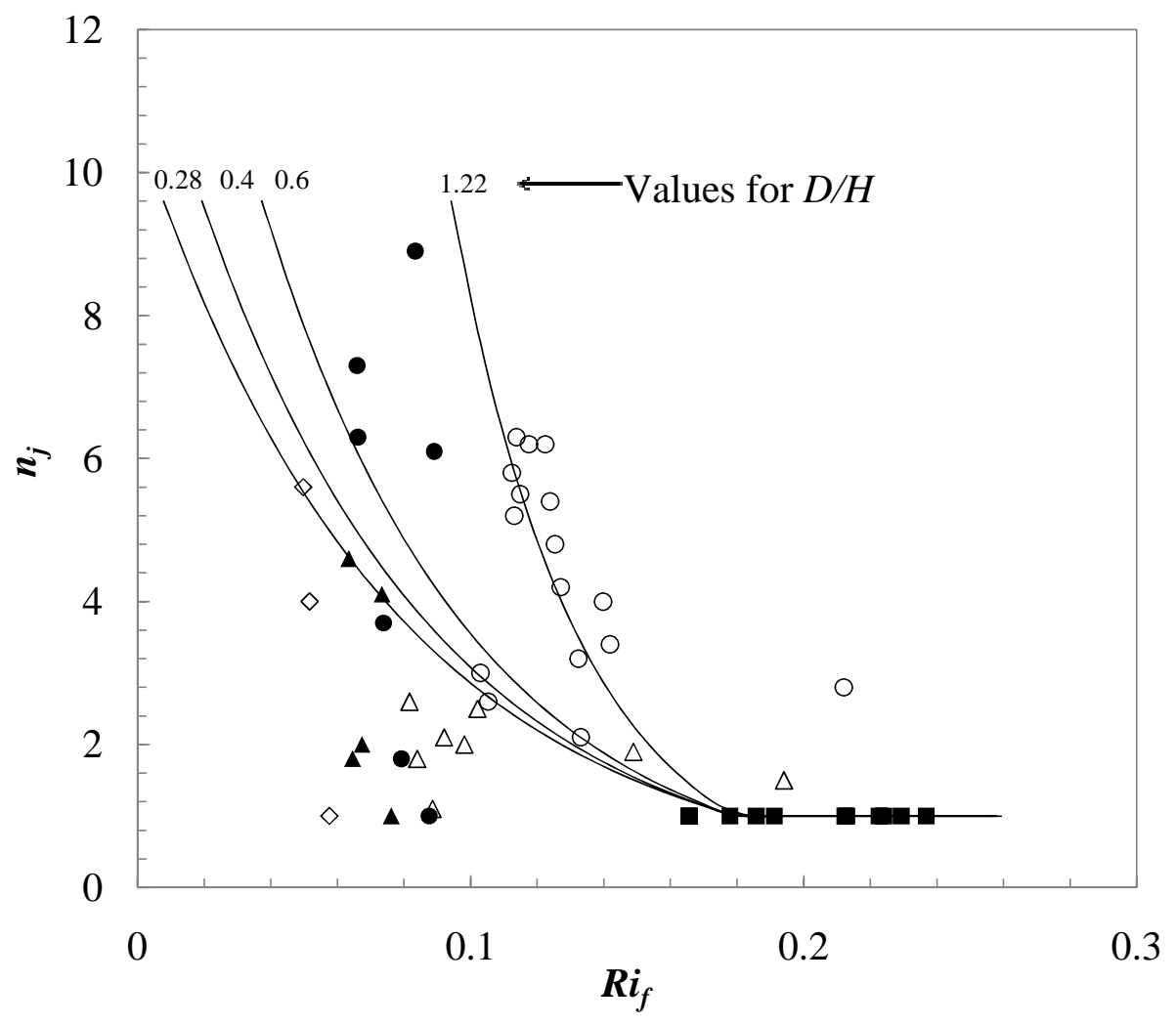

Figure 3. Variation of ceiling jet mixing with Richardson number at the flame tip. Values for D/H: - 4.27, 3.2, 1.28 (Kokkala); ○ 1.22 (Gross), 1.4, 1.0 (You); $\Delta 0.67$ (You), 0.64 (Kokkala); $0.60,0.52$ (You); $\boldsymbol{\Delta} 0.46,0.40$ (You); $\diamond 0.29,0.28$ (You); —— Theory. 


\section{Chapter 3: Results and Analyses}

\subsection{Results for Flame Length, $r_{f}$}

The solution presented in the previous chapter, while in algebraic form, does require iteration to obtain a value for the radial flame length. The essential input parameters are the diameter of the fire, $D$; the height of the ceiling, $H$; and the firepower, $\dot{Q}$. These can be arranged into a dimensionless functionality:

$r_{f} / H=\operatorname{function}\left(Q_{D}^{*}, D / H\right), Q_{D}^{*}=\dot{Q} /\left(\rho_{\infty} c_{p} T_{\infty} g^{1 / 2} D^{5 / 2}\right)$, or equivalent.

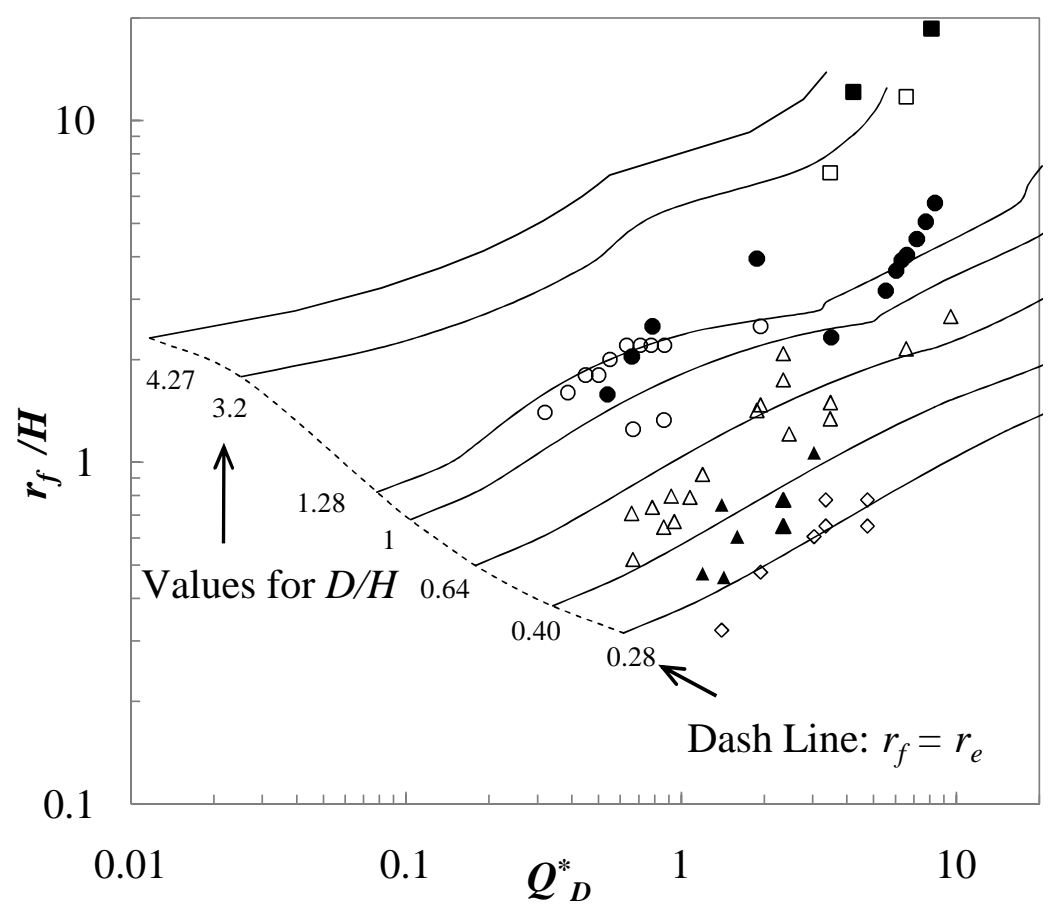

Figure 4. Theoretical results compared to data for radial flame length. Values for $D / H$ : 4.27 (Kokkala); 3.2 (Kokkala); • 1.4 (You), 1.28 (Kokkala); ○ 1.22 (Gross), 1.0 (You); $\Delta 0.66$ (Heskestad), 0.64 (Kokkala), 0.67, 0.60, 0.52 (You); $\boldsymbol{\Delta}$ 0.46, 0.40 (You), 0.40 (Heskestad); $\diamond$ $0.29,0.28$ (You), 0.32, 0.27 (Heskestad); —— Theory; - - $r_{f}=r_{e}$. 
Figure 4 displays the computed results based on Eqns. (38 \& 39) as solid lines, and the associated data. As $D / H$ is large, $3-4$, the ceiling flame becomes very long and the flame is more laminar-like. In general, the radial flame length normalized with the ceiling height, increases as $Q^{*}{ }_{D}$ increases and $D / H$ increases. The theory also indicates the locus of $r_{f}=r_{\mathrm{e}}$ for which the ceiling flame just fills the impingement region. It is tedious to compare the computed solution to the precise corresponding data in Figure 4. Therefore Figure 5 presents a direct comparison. Most of the results fall into -10 to $+25 \%$ accuracy. Full computations are presented in Appendix D.

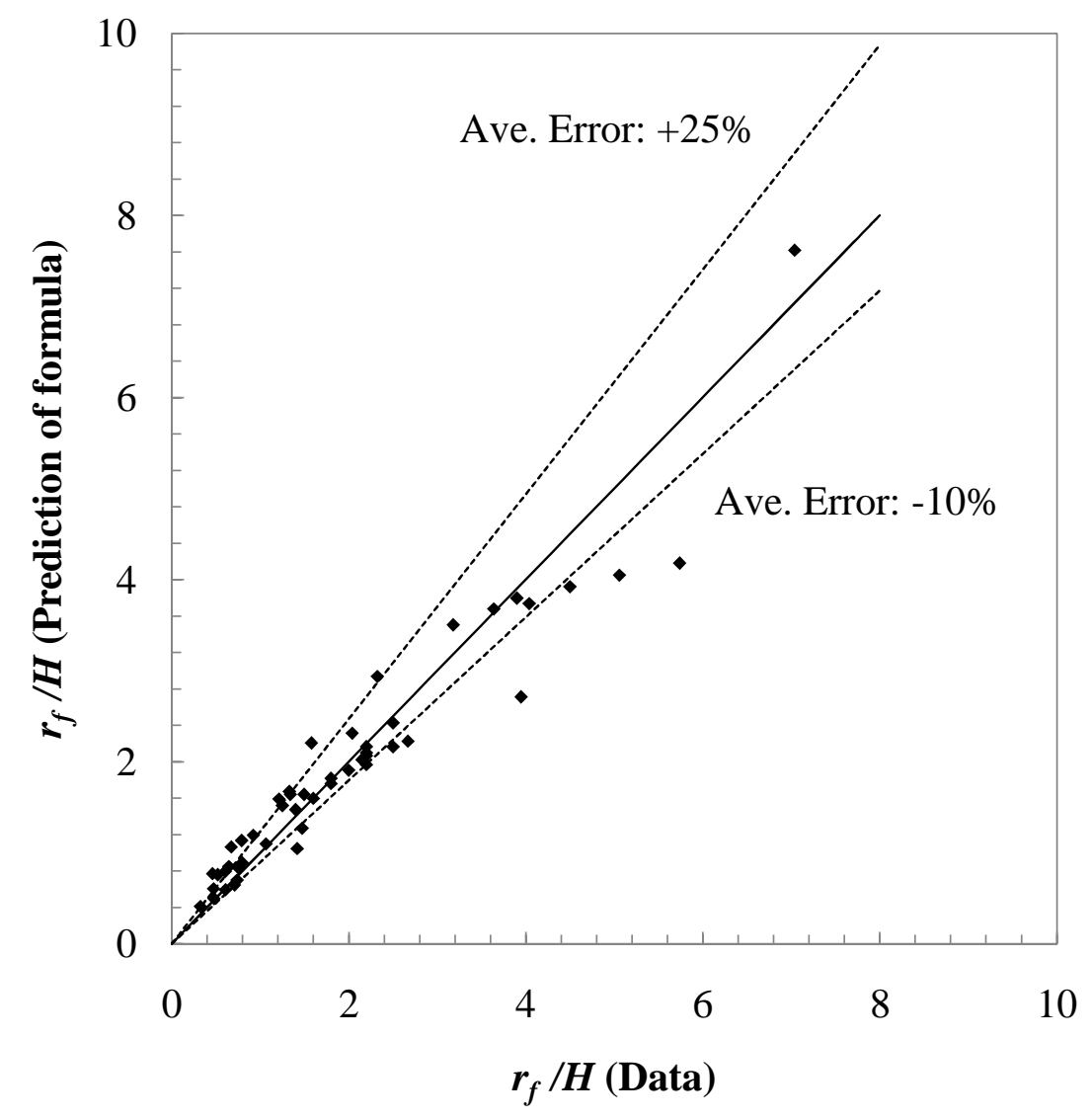

Figure 5. Direct comparison of predicted and experimental results. 


\subsection{Simple Correlation for Flame Length, $r_{f}$}

The objective of this work was to obtain an analytic result, but the solution does not lend itself to such a result. However, the accuracy of the computations suggests that a correlation developed from the theory might prove useful. This was done with a surprising outcome. The effect of $\mathrm{D} / \mathrm{H}$ disappeared from the result. It was found that a good equation for the radial flame length, when the vertical free flame height is computed to be greater than the ceiling, is

$r_{f} / D=1.62 Q_{D}^{* 2 / 5}, H_{f} \geq H$

The corresponding free flame height expression from Grove and Quintiere [1] (see Appendix B) is

$H_{f} / D=6.17 Q_{D}^{* 2 / 5}-1.83$, for $Q_{D}^{*}>0.1$

Figure 6 presents the results for the simple correlation of Eq. (39). The accuracy is similar to the direct computation, so this is a valuable simple equation. Its lack of dependence on $D / H$ might be

partially explained by examining the sketch in Figure 7 . As $D / H$ varies from about 0.2 to 1 , there is no change of $r_{f} / D$ as $Q^{*}$ stays constant and the overall flame length stays nearly the same. 


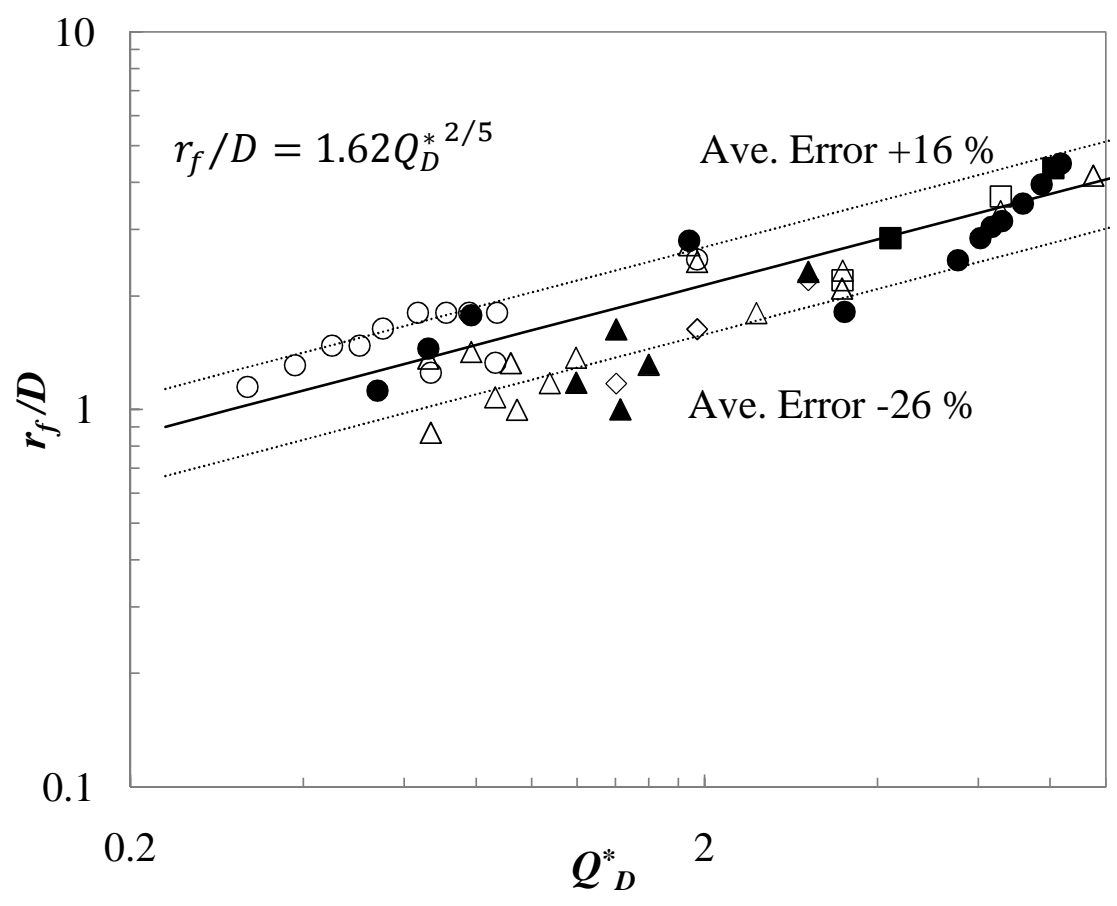

Figure 6. Simple correlation. Values for $D / H:-4.27$ (Kokkala); $\square 3.2$ (Kokkala); • 1.4 (You), 1.28 (Kokkala); ○ 1.22 (Gross), 1.0 (You); $\Delta 0.64$ (Kokkala), 0.67, 0.60, 0.52 (You); $\boldsymbol{\Delta}$ 0.46, 0.40 (You); $\diamond 0.29,0.28$ (You); —— Theory; ---- Error.

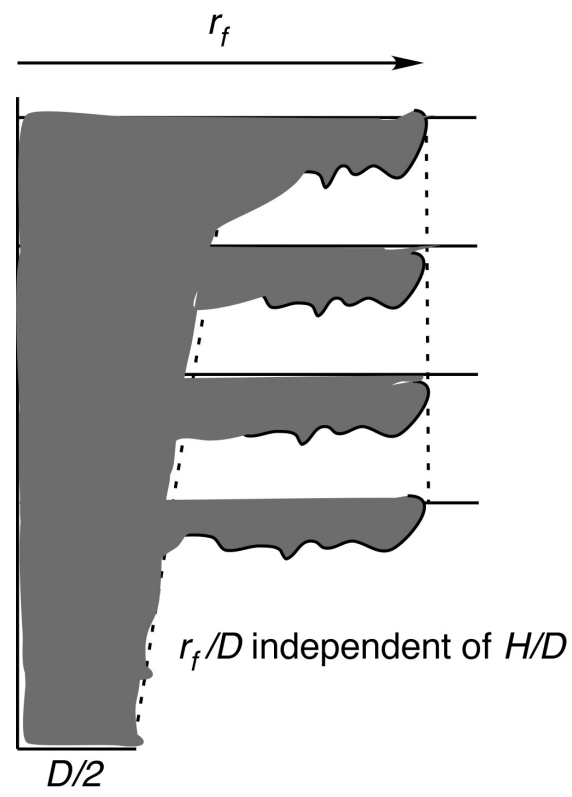

Figure 7. Illustration showing $r_{f}$ is independent of $H$ for given $\dot{Q}$ and $D$. 
The original correlation of You and Faeth [2] combined the free flame length with the actual ceiling height. Their result would still contain a $\mathrm{D} / \mathrm{H}$ effect as they give

$r_{f} / D=0.502\left(\left(H_{f}-H\right) / D\right)^{0.957}$

and substituting Eq. (41) would not eliminate $D / H$. So their correlation cannot be put into the form of Eq. (37). To evaluate its merits over the ensemble of data in Table 1, Figure 8 is plotted using Eqns. (40 \& 41). The results indicate accuracy within 37\%, somewhat higher than that given by the new correlation of Eq. (40) at $26 \%$. Both correlations are roughly linear with the free flame height.

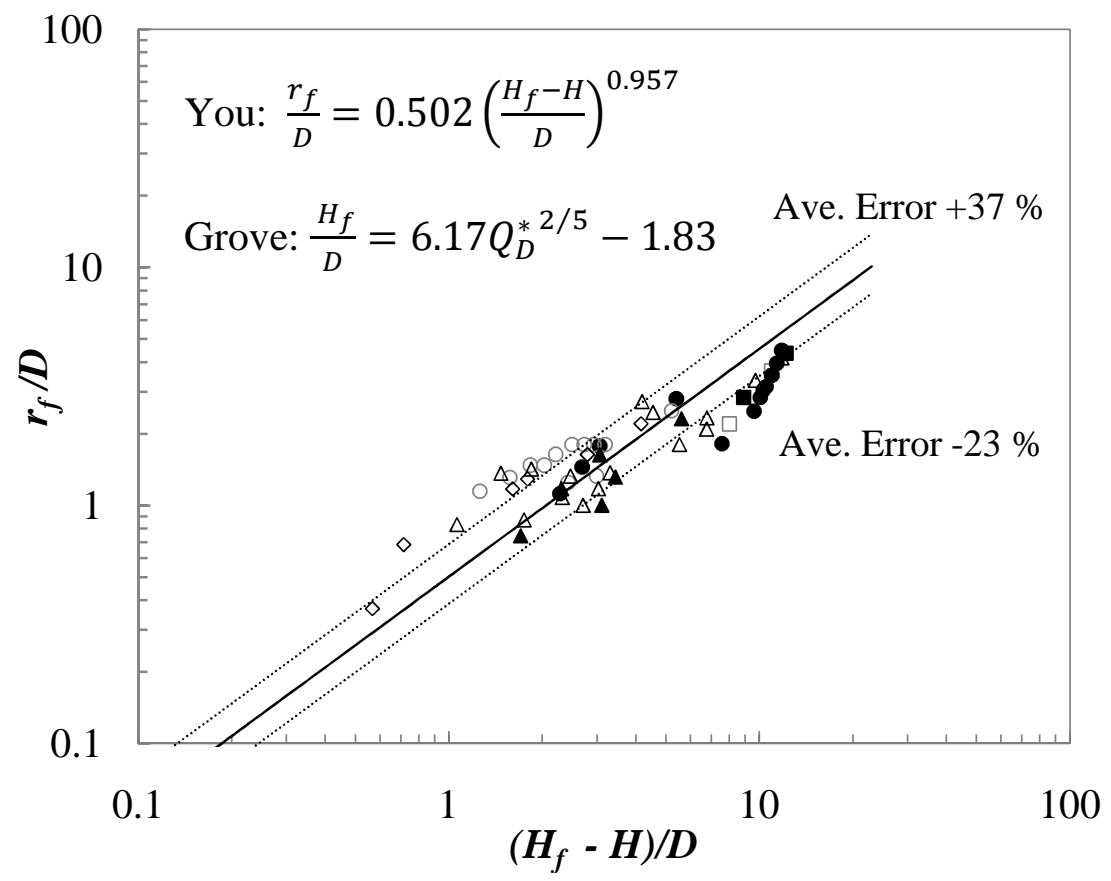

Figure 8. Radial flame length in terms of free flame height. Values for $D / H: \mathbf{\square} 4.27$ (Kokkala); $\square$ 3.2 (Kokkala); • 1.4 (You), 1.28 (Kokkala); ○ 1.22 (Gross), 1.0 (You); $\Delta 0.64$ (Kokkala), 0.67, 0.60, 0.52 (You); $\boldsymbol{\Delta}$ 0.46, 0.40 (You); $\diamond 0.29,0.28,0.23$ (You); —— Theory; ----- Error. 
We seek to relate the flame radial length under the ceiling, $r_{f}$, to the free flame height with the absence of a ceiling, $H_{f}$. Instead of using the free flame height formula of Eq. (41) which was adjusted to fit data of You, we choose to use a more widely known formula provided by Heskestad [14] (see Appendix A):

$H_{f} / D=15.6 Q_{D}^{* 2 / 5} \lambda^{-3 / 5}-1.02$

or the same formula with $\lambda=9.97$

$H_{f} / D=3.93 Q_{D}^{* 2 / 5}-1.02$

Figure 9, combining formula of Eq. (43) for $H_{f}$ and simple correlation of Eq. (40) for $r_{f}$, illustrates how the two are related in application.

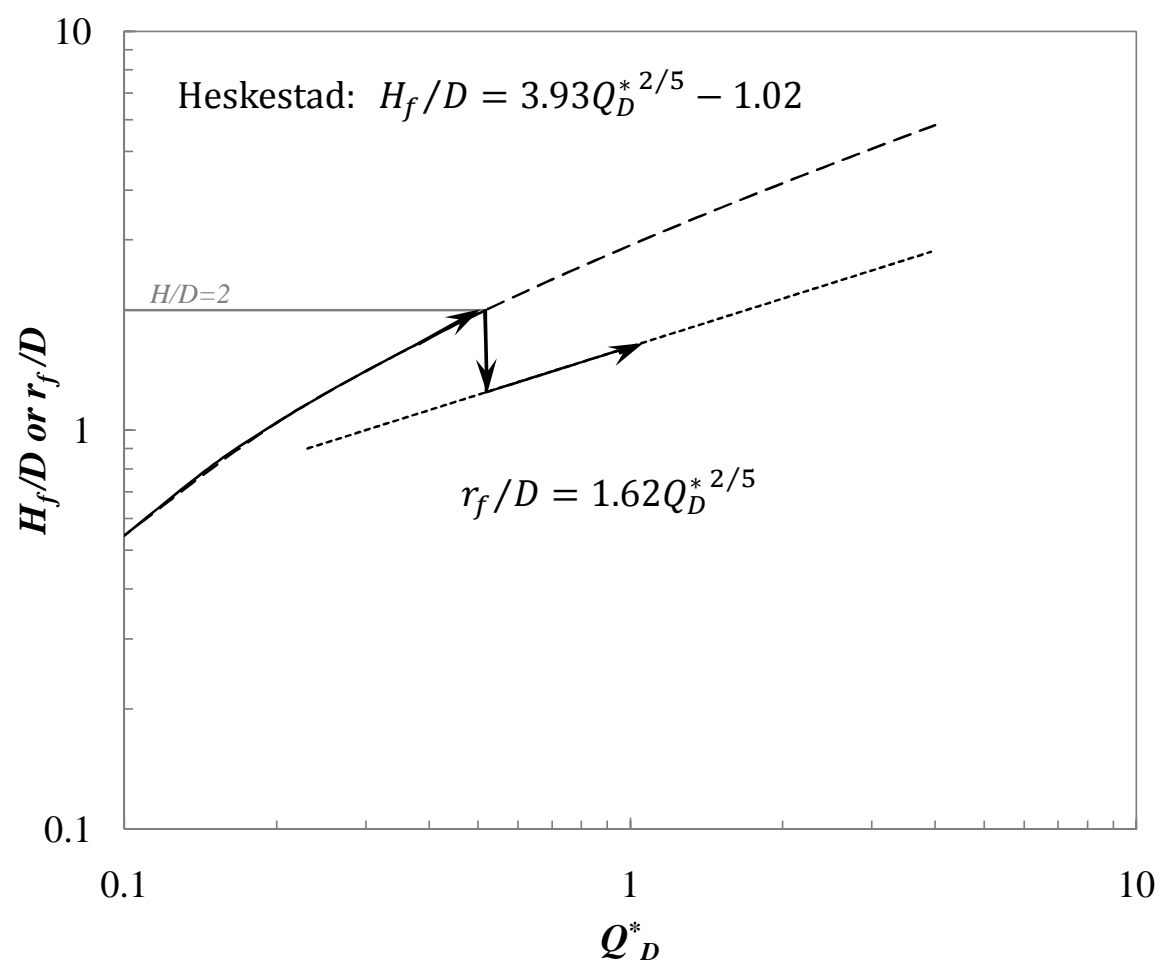

Figure 9. Free flame height and radial flame length. 
Consider a room with ceiling height $D / H=0.5$ (or $H / D=2$ in the figure) as an example. For fire power $Q^{*}$ small, $H_{f} / D<H / D$, i.e. before fire reaches the ceiling, the free flame height follows Eq. (43). For $Q^{*}$ large, $H_{f} / D>H / D$, i.e. after fire hits the ceiling, the flame radial length under the ceiling follows Eq. (40). 


\section{Chapter 4: Summary and Conclusions}

An integral model for the ceiling jet flame coupled with known results for the fire plume region enabled a solution for the radial flame extension. This solution needed to introduce an empirical relationship for the ratio of air entrained to stoichiometric air. That ratio was found to vary from about 1 for "long" flames to ideally 9.6 for flame tip just extending from the plume region. This represents the range of laminar to fully turbulent regimes for the ceiling jet flame. Further study should examine these mixing aspects.

The simple correlation of You and Faeth [2] was examined for a wider database. It showed similar accuracy to a simple correlation derived from the current study. While both show the radial flame extension is nearly linear with the free vertical flame height, the latter correlation showed no dependence on $\mathrm{D} / \mathrm{H}$. 


\section{Appendices}

\section{Appendix A: Data and Calculations for Ceiling Jet Mixing Ratio, $n_{j}$}

\section{A.1. Data}

Data and calculations are presented in the Table 2 below. The left part of the table is experimental data, obtained from previous investigators (also see Table 1). The right part of the table shows calculations using the theories derived in this study. Data is arranged in an order so that $D / H$ decreases.

You and Faeth [2] used several varieties of fuels, including methanol, ethanol, propanol and pentane, but in small scale and at low heat flow rates $(\dot{Q}<10 \mathrm{~kW})$.

Kokkala and Rinkinen [5] conducted small scale experiments. Propane and natural gas flames in the range of $2.9 \mathrm{~kW}$ to $11 \mathrm{~kW}$ on a burner of a diameter of $0.64 \mathrm{~m}$ were used. We extracted their data from a figure using a graph digitizer software, and that is why $r_{f}$ data of many decimal places appear.

Gross [4] conducted large scale experiments and measured both axisymmetric and corner-wall-ceiling configurations for a range of energy supply rates up to $400 \mathrm{~kW}$ and burnerto-ceiling heights up to $2.3 \mathrm{~m}$. Flame length observations were of four types: Visual; $35 \mathrm{~mm}$ photograph, instantaneous - short exposure and extended exposure; and video camera. In our study we used their data from video camera.

Data from You \& Faeth, Kokkala \& Rinkinen, and Gross is listed in the first part of Table 2, and is directly used in investigating the mixing ratio $n_{j}$ and the flame length calculations. 
A few data of flame lengths, $r_{f}$, may have been very slightly adjusted in order to complete the iteration calculations that will be discussed later.

Heskestad and Hamada [9] made observations of the mean flame length in some cases in their experiments where the flames impacted the ceiling. They did not give direct values for flame lengths. Instead, they found that the ratio of the mean flame length, $r_{f}$, to the flame portion intercepted by the ceiling, $H_{f}-H$, ranged from 0.88 to 1.05 , averaging to 0.95 . Their data is listed in the second part of Table 2. For each case, we listed both maximum $\left(r_{f}=1.05\left(H_{f}-H\right)\right)$ and minimum $\left(r_{f}=0.88\left(H_{f}-H\right)\right)$ values from calculations. The calculations are only on the purpose of including their data ranges in Figure 4. Their data was not used anywhere else in this study.

Our theory assumes fire grows large enough to pass the turning region. For some cases where the flame hits the ceiling but is not large enough to pass the turning region, i.e. $r_{f}<r_{e}$, calculation results were abandoned because our theory does not apply to such cases.

\section{A.2. Calculations for Ceiling Jet Mixing Ratio, $n_{j}$}

Eq. (37) gives a relationship between the overall energy release rate and the radial flame length. The only unknown is $n_{j}$, the ratio of actual air entrained to the stoichiometric air. The purpose of Table 2 is to use Eq. (37) and experimental data to calculate an empirical value for $n_{j}$. After that we seek to find a reasonable correlation for $n_{j}$, so that it becomes a known parameter and thus can be used to calculate the relation between the overall energy release rate and the radial flame length in Eq. (37). The latter part of calculations is presented in Appendix D and Table 3.

Several things involved in calculating $n_{j}$ need to be noted: 
1) When using Eq. (37), we decided to use $r_{f}$ as the input to calculate $\dot{Q}$, rather than to use $\dot{Q}$ to calculate $r_{f}$, because $h_{f}, v_{f}$ and $n_{j}$ are all functions of $r_{f}$. As a result, when calculating the values for $n_{j}$, we did so by setting a value for each $n_{j}$ so that in the end the calculated heat release rate, $Q_{D}{ }_{2}$, is as close as possible to the original heat release rate from the experiments, $Q_{D}{ }^{*}{ }_{1}$.

2) In order to obtain values for $h_{f}, v_{f}, \theta_{f}$ and $R i_{f}$ at the flame tip, the value for $E$ at the flame tip, $E_{f}$, is needed. However, $E_{f}$ is a function of $R i_{f}$, which depends on $h_{f}, v_{f}, \theta_{f}$. Therefore iteration is needed. $h_{f}, v_{f}, \theta_{f}$ and $R i_{f}$ are first calculated using $E_{e}$, and then a value for $E_{f}$ can be obtained through this $E_{e} . E_{e}$ is then reset to the same value as $E_{f}$, which will in turn change values for all the parameters. After iterations when $E_{e}$ finally reaches the same value as $E_{f}$, values for $h_{f}, v_{f}, \theta_{f}$ and $R i_{f}$ are carried out.

3) The model assumes that combustion rate is controlled by air entrainment, and the actual air entrained into the ceiling jet is in excess of the air needed for combustion (e.g., the ratio of air entrained to the fire plume to the stochiometric air is $n_{p}=9.6$ [1]). The assumption determines that the value of $n_{j}$ cannot go below 1 . Table 2 presented the original calculation results. Some of the results show $n_{j}$ even approaches 0 . We consider those calculation results not physical. Therefore, when we present $n_{j}$ results in Figure 3 in order to investigate its behavior, $n_{j}$ is reset to 1 for data sets where $n_{j}<1$, and calculations were performed again with $n_{j}=1$.

The calculation results for $n_{j}$ are presented in Table 2. The first four columns $(\dot{Q}, D, H, r f)$ are raw data from other investigators [2, 4, $5 \& 9]$. The rest are the calculation results from this study. 
As Ri controls the nature of the turbulent mixing, we chose to investigate $n j$ as a function of $\mathrm{Ri}$. The results for $n_{j}$ and $\mathrm{Ri}_{f}$ are marked in Figure 3. As observations made in Chapter 2.8 has stated, the variation of $n_{j}$ ranged from about 1 to 10 . There is a trend that shows $n j$ decreases from values near 10 to values near 1 . We expect for those short radial flames where fire hits the ceiling and stops right at the ceiling impingement (i.e. $r_{f}=r_{e}$ and $R i_{f}=\mathrm{Ri}_{e}$ ), $n_{j}$ is likely to be close to the turbulent mixing ratio in the plume, 9.6, while in the long laminar radial flames, this value would approach 1. It is generally expected that as the $\mathrm{Ri}$ increases to a value of about 0.3 , turbulent mixing is suppressed and laminarization of the jet can occur [12], but no satisfactory theoretical estimate of critical Ri has yet been made. By examining the data trend, we determine the critical Ri to be 0.23 for this study. A family of curves pertains to $D / H$ is also observed. With the starting position $\left(n_{j}=9.6\right)$ and the end position $\left(n_{j}=1\right)$ fixed, $\varphi$ containing $D / H$ is correlated as

$\varphi(D / H)=\ln 9.6 /\left(0.23-\mathrm{Ri}_{e}^{\prime}\right)$ or equivalently, $\varphi(D / H)=2.26 /(0.197-0.091 D / H)$

A constant $C_{n}$ is introduced to fit data. The model for $n_{j}$ vs. $\mathrm{Ri}_{f}$ is thus made as

$n_{j}=9.6 \exp \left[\varphi\left(\left(\mathrm{Ri}_{e}-\mathrm{Ri}_{f}-C_{n}\right)\right]\right.$

and $C_{n}$ is found to be 0.05 .

Therefore we have

$n_{j}=9.6 \exp \left[-\left(2.26\left(\mathrm{Ri}_{f}-\mathrm{Ri}_{e}+0.05\right) /(0.197-0.091 D / H)\right)\right]$, for $\mathrm{Ri}_{f} \leq 0.18$

$n_{j}=1$, for $\mathrm{Ri}_{f} \geq 0.18$ 
The above process in the formulation of $n_{j}$ shows that though it is evaluated from an empirical fit, it has its physical base. 
Table 2. Data and calculations for ceiling jet mixing ratio, $n_{j}$

\begin{tabular}{|c|c|c|c|c|c|c|c|c|c|c|c|c|c|c|c|c|c|c|c|c|c|c|}
\hline$\dot{Q}(\mathrm{~kW})$ & $D(\mathrm{~m})$ & $H(\mathrm{~m})$ & $r_{f}(\mathrm{~m})$ & $r_{f}^{\prime} / r_{e}{ }^{\prime}$ & $D / H$ & $r_{e}{ }^{\prime}$ & $v_{e}^{\prime}$ & $h e^{\prime}$ & $\theta_{e}$ & $R i_{e}$ & $E_{e}$ & $r_{f}^{\prime}$ & $h_{f}^{\prime}$ & $v_{f}^{\prime}$ & $\theta_{f}$ & $n_{j}$ & $R i_{f}$ & $E_{f}$ & $m_{p}^{*}$ & $m_{j}^{*}$ & $Q_{D_{2}}^{*}$ & $Q_{D_{1}}^{*}$ \\
\hline 4.888 & 0.064 & 0.015 & 0.182 & 5.25 & 4.27 & 2.31 & 2.02 & 0.90 & 1.91 & 0.422 & 0.001 & 12.13 & 0.24 & 1.49 & 6.84 & 0.0 & 0.739 & 0.001 & 0.011 & 0.014 & 3.58 & 4.21 \\
\hline 9.40 & 0.064 & 0.015 & 0.279 & 8.04 & 4.27 & 2.31 & 2.02 & 0.90 & 1.91 & 0.422 & 0.000 & 18.60 & 0.20 & 1.11 & 7.85 & 0.0 & 1.297 & 0.000 & 0.011 & 0.001 & 4.20 & 8.11 \\
\hline 4.032 & 0.064 & 0.02 & 0.1408 & 3.96 & 3.20 & 1.78 & 2.02 & 0.69 & 1.91 & 0.325 & 0.004 & 7.04 & 0.23 & 1.62 & 4.74 & 0.2 & 0.423 & 0.004 & 0.024 & 0.054 & 2.70 & 3.48 \\
\hline 7.621 & 0.064 & 0.02 & 0.235 & 6.60 & 3.20 & 1.78 & 2.02 & 0.69 & 1.91 & 0.325 & 0.001 & 11.75 & 0.17 & 1.28 & 6.72 & 0.1 & 0.697 & 0.001 & 0.024 & 0.026 & 4.31 & 6.57 \\
\hline 2.26 & 0.107 & 0.076 & 0.12 & 1.79 & 1.41 & 0.88 & 2.02 & 0.34 & 1.91 & 0.161 & 0.022 & 1.58 & 0.22 & 1.86 & 2.07 & 2.1 & 0.133 & 0.022 & 0.068 & 0.097 & 0.53 & 0.54 \\
\hline 2.77 & 0.107 & 0.076 & 0.155 & 2.31 & 1.41 & 0.88 & 2.02 & 0.34 & 1.91 & 0.161 & 0.022 & 2.04 & 0.19 & 1.73 & 2.03 & 3.2 & 0.132 & 0.022 & 0.068 & 0.187 & 0.65 & 0.66 \\
\hline 3.29 & 0.107 & 0.076 & 0.19 & 2.83 & 1.41 & 0.88 & 2.02 & 0.34 & 1.91 & 0.161 & 0.022 & 2.50 & 0.18 & 1.60 & 2.00 & 4.0 & 0.140 & 0.022 & 0.068 & 0.282 & 0.77 & 0.78 \\
\hline 7.89 & 0.107 & 0.076 & 0.3 & 4.47 & 1.41 & 0.88 & 2.02 & 0.34 & 1.91 & 0.161 & 0.015 & 3.95 & 0.15 & 1.32 & 2.29 & 2.8 & 0.201 & 0.015 & 0.068 & 0.481 & 1.78 & 1.88 \\
\hline 4.07 & 0.064 & 0.05 & 0.11615 & 2.84 & 1.28 & 0.82 & 2.02 & 0.32 & 1.91 & 0.150 & 0.014 & 2.32 & 0.15 & 1.68 & 3.43 & 0.6 & 0.185 & 0.014 & 0.075 & 0.207 & 3.52 & 3.51 \\
\hline 6.414 & 0.064 & 0.05 & 0.15905 & 3.88 & 1.28 & 0.82 & 2.02 & 0.32 & 1.91 & 0.150 & 0.010 & 3.18 & 0.13 & 1.51 & 4.33 & 0.5 & 0.240 & 0.010 & 0.075 & 0.278 & 5.62 & 5.53 \\
\hline 7.013 & 0.064 & 0.05 & 0.182 & 4.44 & 1.28 & 0.82 & 2.02 & 0.32 & 1.91 & 0.150 & 0.009 & 3.64 & 0.12 & 1.43 & 4.54 & 0.5 & 0.262 & 0.009 & 0.075 & 0.306 & 6.17 & 6.05 \\
\hline 7.655 & 0.064 & 0.05 & 0.202 & 4.93 & 1.28 & 0.82 & 2.02 & 0.32 & 1.91 & 0.150 & 0.008 & 4.04 & 0.11 & 1.37 & 4.69 & 0.5 & 0.284 & 0.008 & 0.075 & 0.327 & 6.59 & 6.60 \\
\hline 7.336 & 0.064 & 0.05 & 0.195 & 4.76 & 1.28 & 0.82 & 2.02 & 0.32 & 1.91 & 0.150 & 0.008 & 3.90 & 0.11 & 1.39 & 4.63 & 0.5 & 0.275 & 0.008 & 0.075 & 0.319 & 6.43 & 6.32 \\
\hline 8.322 & 0.064 & 0.05 & 0.225 & 5.49 & 1.28 & 0.82 & 2.02 & 0.32 & 1.91 & 0.150 & 0.007 & 4.50 & 0.11 & 1.29 & 4.88 & 0.5 & 0.320 & 0.006 & 0.075 & 0.353 & 7.13 & 7.17 \\
\hline 8.97 & 0.064 & 0.05 & 0.253 & 6.18 & 1.28 & 0.82 & 2.02 & 0.32 & 1.91 & 0.150 & 0.006 & 5.06 & 0.10 & 1.21 & 4.91 & 0.5 & 0.346 & 0.005 & 0.075 & 0.357 & 7.19 & 7.73 \\
\hline 9.688 & 0.064 & 0.05 & 0.287 & 7.01 & 1.28 & 0.82 & 2.02 & 0.32 & 1.91 & 0.150 & 0.004 & 5.74 & 0.10 & 1.13 & 5.43 & 0.4 & 0.409 & 0.004 & 0.075 & 0.323 & 8.14 & 8.35 \\
\hline 104 & 0.61 & 0.5 & 0.7 & 1.77 & 1.22 & 0.79 & 2.02 & 0.31 & 1.91 & 0.144 & 0.025 & 1.40 & 0.20 & 1.85 & 1.91 & 5.2 & 0.113 & 0.025 & 0.080 & 0.123 & 0.32 & 0.32 \\
\hline 126 & 0.61 & 0.5 & 0.8 & 2.03 & 1.22 & 0.79 & 2.02 & 0.31 & 1.91 & 0.144 & 0.025 & 1.60 & 0.19 & 1.78 & 1.89 & 5.8 & 0.112 & 0.025 & 0.080 & 0.176 & 0.39 & 0.39 \\
\hline 146 & 0.61 & 0.5 & 0.9 & 2.28 & 1.22 & 0.79 & 2.02 & 0.31 & 1.91 & 0.144 & 0.025 & 1.80 & 0.18 & 1.72 & 1.87 & 6.3 & 0.114 & 0.025 & 0.080 & 0.232 & 0.45 & 0.45 \\
\hline 163 & 0.61 & 0.5 & 0.9 & 2.28 & 1.22 & 0.79 & 2.02 & 0.31 & 1.91 & 0.144 & 0.025 & 1.80 & 0.18 & 1.72 & 1.90 & 5.5 & 0.115 & 0.025 & 0.080 & 0.230 & 0.50 & 0.50 \\
\hline 179 & 0.61 & 0.5 & 1 & 2.53 & 1.22 & 0.79 & 2.02 & 0.31 & 1.91 & 0.144 & 0.025 & 2.00 & 0.17 & 1.65 & 1.87 & 6.2 & 0.118 & 0.025 & 0.080 & 0.289 & 0.55 & 0.55 \\
\hline 206 & 0.61 & 0.5 & 1.1 & 2.79 & 1.22 & 0.79 & 2.02 & 0.31 & 1.91 & 0.144 & 0.024 & 2.20 & 0.17 & 1.59 & 1.86 & 6.2 & 0.122 & 0.024 & 0.080 & 0.345 & 0.64 & 0.63 \\
\hline 231 & 0.61 & 0.5 & 1.1 & 2.79 & 1.22 & 0.79 & 2.02 & 0.31 & 1.91 & 0.144 & 0.024 & 2.20 & 0.17 & 1.59 & 1.90 & 5.4 & 0.124 & 0.024 & 0.080 & 0.341 & 0.71 & 0.71 \\
\hline 253 & 0.61 & 0.5 & 1.1 & 2.79 & 1.22 & 0.79 & 2.02 & 0.31 & 1.91 & 0.144 & 0.023 & 2.20 & 0.16 & 1.60 & 1.94 & 4.8 & 0.125 & 0.023 & 0.080 & 0.337 & 0.78 & 0.78 \\
\hline
\end{tabular}




\begin{tabular}{|c|c|c|c|c|c|c|c|c|c|c|c|c|c|c|c|c|c|c|c|c|c|c|}
\hline 283 & 0.61 & 0.5 & 1.1 & 2.79 & 1.22 & 0.79 & 2.02 & 0.31 & 1.91 & 0.144 & 0.023 & 2.20 & 0.16 & 1.60 & 1.98 & 4.2 & 0.127 & 0.023 & 0.080 & 0.332 & 0.87 & 0.87 \\
\hline 1.19 & 0.076 & 0.076 & 0.095 & 1.84 & 1.00 & 0.68 & 2.02 & 0.26 & 1.91 & 0.124 & 0.026 & 1.25 & 0.17 & 1.83 & 2.01 & 3.0 & 0.103 & 0.026 & 0.100 & 0.172 & 0.67 & 0.67 \\
\hline 1.54 & 0.076 & 0.076 & 0.101 & 96 & 1.00 & 0.68 & 2.02 & 26 & & & 25 & & & & 2.06 & 2.6 & & 25 & 100 & 98 & 86 & 86 \\
\hline 3.46 & 076 & 0.076 & 0.19 & 3.68 & 1.00 & 0.68 & 2.02 & 0.26 & 1.91 & 0.124 & 0.021 & 2.50 & 0.13 & 1.41 & 2.13 & 3.4 & 0.142 & 0.021 & 0.100 & 0.631 & 1.95 & 1.94 \\
\hline 0.62 & 051 & 0.076 & 0.051 & 1.30 & 0.67 & 0.51 & 2.02 & 20 & 1.91 & 0.094 & 0.027 & 0.67 & 0.16 & 1.96 & 2.08 & 1.1 & 0.089 & 0.027 & 0.162 & 0.086 & 0.95 & 0.94 \\
\hline 707 & 0.051 & 0.076 & 0.06 & .53 & 0.67 & 0.51 & 2.02 & 0.20 & 1.91 & 0.094 & 0.028 & 0.79 & 0.15 & 1.90 & 2.07 & 1.8 & 0.084 & 0.028 & 0.162 & .168 & 1.10 & 1.08 \\
\hline 0.786 & 0.051 & 0.076 & 0.07 & 1.79 & 0.67 & 0.51 & 2.02 & 0.20 & 1.91 & 0.094 & 0.029 & 0.92 & 0.13 & 1.83 & 2.05 & 2.6 & 0.082 & 0.029 & 0.162 & 0.272 & 1.21 & 1.20 \\
\hline 1.62 & 0.051 & 0.076 & 0.092 & 2.35 & 0.67 & 0.51 & 2.02 & 0.20 & 1.91 & 0.094 & 0.026 & 1.21 & 0.12 & 1.69 & 2.26 & 2.1 & 0.092 & 0.026 & 0.162 & 0.493 & 2.51 & 2.46 \\
\hline 4.04 & 0.064 & 0.1 & 0.150 & 3.00 & 0.64 & 0.50 & 2.02 & 0.19 & 1.91 & 0.091 & 0.025 & 1.50 & 0.10 & 1.53 & 2.28 & 2.5 & 0.102 & 0.025 & 0.172 & 0.825 & 3.47 & 3.48 \\
\hline 4.03 & 0.064 & 0.1 & 0.134 & 2.69 & 0.64 & 0.50 & 2.02 & 0.19 & 1.91 & 0.091 & 0.025 & 1.34 & 0.11 & 1.61 & 2.37 & 2.0 & 0.098 & 0.025 & 0.172 & 0.665 & 3.49 & 3.47 \\
\hline 7.612 & 0.064 & 0.1 & 21485 & 4.31 & 0.64 & 0.50 & 2.02 & 0.19 & 1.91 & 0.091 & 0.018 & 2.15 & 0.09 & 1.31 & 2.73 & 1.9 & 0.149 & 0.018 & 0.172 & 1.231 & 6.64 & 6.56 \\
\hline 11.04 & 0.064 & 0.1 & 2669 & 5.35 & 0.64 & 0.50 & 2.02 & 0.19 & 1.91 & 0.091 & 0.014 & 2.67 & 0.09 & 1.18 & 3.18 & 1.5 & 194 & 014 & 172 & 379 & 9.34 & 9.52 \\
\hline 1.19 & 0.076 & 0.127 & 0.066 & 1.09 & 0.60 & 0.48 & 2.02 & 0.19 & 1.91 & 0.087 & 0.028 & 0.52 & 0.17 & 2.00 & 2.01 & 0.6 & 0.088 & 0.028 & 0.188 & 0.026 & 0.67 & 0.67 \\
\hline 1.54 & 0.076 & 0.127 & 0.082 & 1.35 & 0.60 & 0.48 & 2.02 & 0.19 & 1.91 & 0.087 & 0.029 & 0.65 & 0.15 & 1.94 & 2.02 & 1.8 & 0.079 & 0.029 & 0.188 & 0.123 & 0.88 & 0.86 \\
\hline 1.64 & 0.076 & 0.127 & 0.101 & 1.66 & 0.60 & 0.48 & 2.02 & 0.19 & 1.91 & 0.087 & 0.030 & 0.80 & 0.13 & 1.86 & 1.96 & 3.7 & 0.074 & 0.030 & 0.188 & 0.267 & 0.91 & 0.92 \\
\hline 3.46 & 0.076 & 0.127 & 0.187 & 3.08 & 0.60 & 0.48 & 2.02 & 0.19 & 1.91 & 0.087 & 0.028 & 1.47 & 0.10 & 1.48 & 1.85 & 6.1 & 0.089 & 0.028 & 0.188 & 1.069 & 1.94 & 1.94 \\
\hline $2.26^{*}$ & 0.107 & 0.206 & 0.089 & 0.98 & 0.52 & 0.44 & 2.02 & 0.17 & 1.91 & 0.080 & 0.029 & 0.43 & 0.17 & 2.02 & 1.90 & 1.0 & 0.081 & 0.029 & 0.227 & -0.01 & 0.18 & 0.54 \\
\hline 2.77 & 0.107 & 0.206 & 0.146 & 1.62 & 0.52 & 0.44 & 2.02 & 0.17 & 1.91 & 0.080 & 0.032 & 0.71 & 0.12 & 1.86 & 1.88 & 7.3 & 0.066 & 0.032 & 0.227 & 0.309 & 0.66 & 0.66 \\
\hline 3.29 & 0.107 & 0.206 & 0.152 & 1.68 & 0.52 & 0.44 & 2.02 & 0.17 & 1.91 & 0.080 & 0.032 & 0.74 & 0.12 & 1.85 & 1.89 & 6.3 & 0.066 & 0.032 & 0.227 & 0.348 & 0.79 & 0.78 \\
\hline 7.89 & 0.107 & 0.206 & 0.292 & 3.23 & 0.52 & 0.44 & 2.02 & 0.17 & 1.91 & 0.080 & 0.030 & 1.42 & 0.10 & 1.42 & 1.73 & 8.9 & 0.083 & 0.030 & 0.227 & 1.471 & 1.88 & 1.88 \\
\hline 0.366 & 0.035 & 0.076 & 0.035 & 1.13 & 0.46 & 0.41 & 2.02 & 0.16 & 1.91 & 0.075 & 0.029 & 0.46 & 0.15 & 1.99 & 2.09 & 0.5 & 0.076 & 0.029 & 0.269 & 0.057 & 1.41 & 1.43 \\
\hline 0.41 & 0.035 & 0.076 & 0.046 & 1.48 & 0.46 & 0.41 & 2.02 & 0.16 & 1.91 & 0.075 & 0.031 & 0.61 & 0.12 & 1.90 & 2.04 & 2.0 & 0.067 & 0.031 & 0.269 & 0.261 & 1.58 & 1.60 \\
\hline 0.36 & 0.035 & 0.076 & 0.057 & 1.83 & 0.46 & 0.41 & 2.02 & 0.16 & 1.91 & 0.075 & 0.032 & 0.75 & 0.11 & 1.80 & 1.93 & 4.6 & 0.063 & 0.032 & 0.269 & 0.524 & 1.41 & 1.40 \\
\hline 0.778 & 0.035 & 0.076 & 0.081 & 2.60 & 0.46 & 0.41 & 2.02 & 0.16 & 1.91 & 0.075 & 0.030 & 1.07 & 0.09 & 1.59 & 2.00 & 4.1 & 0.073 & 0.030 & 0.269 & 1.130 & 3.03 & 3.03 \\
\hline $0.62 *$ & 0.051 & 0.127 & 0.038 & 0.79 & 0.40 & 0.38 & 2.02 & 0.15 & 1.91 & 0.069 & 0.029 & 0.30 & 0.18 & 2.06 & 1.92 & 9.0 & 0.082 & 0.029 & 0.328 & -0.10 & 0.23 & 0.94 \\
\hline 0.786 & 0.051 & 0.127 & 0.06 & 1.24 & 0.40 & 0.38 & 2.02 & 0.15 & 1.91 & 0.069 & 0.031 & 0.47 & 0.13 & 1.96 & 1.99 & 1.8 & 0.065 & 0.031 & 0.328 & 0.152 & 1.18 & 1.20 \\
\hline $1.19 *$ & 0.076 & 0.26 & 0.026 & 0.31 & 0.29 & 0.33 & 2.02 & 0.13 & 1.91 & 0.059 & 0.019 & 0.10 & 0.39 & 2.09 & 1.93 & 9.0 & 0.172 & 0.019 & 0.539 & -0.24 & 0.28 & 0.67 \\
\hline
\end{tabular}




\begin{tabular}{|c|c|c|c|c|c|c|c|c|c|c|c|c|c|c|c|c|c|c|c|c|c|c|}
\hline $1.54 *$ & 0.076 & 0.26 & 0.028 & 0.33 & 0.29 & 0.33 & 2.02 & 0.13 & 1.91 & 0.059 & 0.020 & 0.11 & 0.36 & 2.09 & 1.93 & 9.0 & 0.159 & 0.020 & 0.539 & -0.26 & 0.27 & 0.86 \\
\hline $1.64 *$ & 0.076 & 0.26 & 0.052 & 0.62 & 0.29 & 0.33 & 2.02 & 0.13 & 1.91 & 0.059 & 0.028 & 0.20 & 0.20 & 2.08 & 1.93 & 9.0 & 0.087 & 0.028 & 0.539 & -0.26 & 0.27 & 0.92 \\
\hline 3.46 & 0.076 & 0.26 & 0.124 & 1.47 & 0.29 & 0.33 & 2.02 & 0.13 & 1.91 & 0.059 & 0.034 & 0.48 & 0.10 & 1.90 & 1.94 & 4.0 & 0.052 & 0.034 & 0.539 & 0.548 & 1.92 & 1.94 \\
\hline 0.36 & 0.035 & 0.127 & 0.041 & 1.02 & 0.28 & 0.32 & 2.02 & 0.12 & 1.91 & 0.058 & 0.032 & 0.32 & 0.12 & 2.02 & 1.97 & 0.3 & 0.059 & 0.032 & 0.594 & 0.020 & 1.41 & 1.40 \\
\hline 0.778 & 0.035 & 0.127 & 0.077 & 1.91 & 0.28 & 0.32 & 2.02 & 0.12 & 1.91 & 0.058 & 0.034 & 0.61 & 0.08 & 1.77 & 1.90 & 5.6 & 0.050 & 0.034 & 0.594 & 1.363 & 3.04 & 3.03 \\
\hline $1.54 *$ & 0.076 & 0.336 & 0.006 & 0.06 & 0.23 & 0.29 & 2.02 & 0.11 & 1.91 & 0.053 & 0.001 & 0.02 & 1.84 & 2.05 & 1.91 & 9.0 & 0.837 & 0.001 & 0.831 & -0.01 & 0.85 & 0.86 \\
\hline $1.64 *$ & 0.076 & 0.336 & 0.032 & 0.33 & 0.23 & 0.29 & 2.02 & 0.11 & 1.91 & 0.053 & 0.021 & 0.10 & 0.33 & 2.10 & 1.93 & 9.0 & 0.144 & 0.021 & 0.831 & -0.43 & 0.39 & 0.92 \\
\hline $3.46^{*}$ & 0.076 & 0.336 & 0.098 & 1.00 & 0.23 & 0.29 & 2.02 & 0.11 & 1.91 & 0.053 & 0.034 & 0.29 & 0.11 & 2.02 & 1.91 & 9.0 & 0.053 & 0.034 & 0.831 & -0.00 & 0.86 & 1.94 \\
\hline $92.6^{*}$ & 0.3 & 0.92 & 0.194304 & 0.62 & 0.33 & & & & & & & & & & & & & & & & & 1.68 \\
\hline 185 & 0.3 & 0.92 & 0.71484 & 2.27 & 0.33 & & & & & & & & & & & & & & & & & 3.35 \\
\hline 185 & 0.3 & 0.92 & 0.599104 & 1.90 & 0.33 & & & & & & & & & & & & & & & & & 3.35 \\
\hline $23.2^{*}$ & 0.15 & 0.56 & 0.14112 & 0.81 & 0.27 & & & & & & & & & & & & & & & & & 2.38 \\
\hline $23.2 *$ & 0.15 & 0.56 & 0.118272 & 0.67 & 0.27 & & & & & & & & & & & & & & & & & 2.38 \\
\hline 46.3 & 0.15 & 0.56 & 0.43512 & 2.48 & 0.27 & & & & & & & & & & & & & & & & & 4.75 \\
\hline 46.3 & 0.15 & 0.56 & 0.364672 & 2.08 & 0.27 & & & & & & & & & & & & & & & & & 4.75 \\
\hline $382 *$ & 0.61 & 1.51 & 0.38052 & 0.66 & 0.40 & & & & & & & & & & & & & & & & & 1.17 \\
\hline 764 & 0.61 & 1.51 & 1.17327 & 2.04 & 0.40 & & & & & & & & & & & & & & & & & 2.35 \\
\hline 764 & 0.61 & 1.51 & 0.983312 & 1.71 & 0.40 & & & & & & & & & & & & & & & & & 2.35 \\
\hline 764 & 0.61 & 0.92 & 1.91268 & 4.07 & 0.66 & & & & & & & & & & & & & & & & & 2.35 \\
\hline 764 & 0.61 & 0.92 & 1.603008 & 3.41 & 0.66 & & & & & & & & & & & & & & & & & 2.35 \\
\hline
\end{tabular}

* Calculation results are abandoned for these data sets because $r_{f}{ }^{\prime} / r_{e}{ }^{\prime} \leq 1$. 


\section{Note:}

$$
\begin{aligned}
& r_{i}^{\prime}=r_{i} / H \\
& h_{i}{ }^{\prime}=h_{i} / H \\
& v_{i}^{\prime}=v_{i} / \sqrt{g H} \\
& \theta_{i}=\left(T_{i}-T_{\infty}\right) / T_{\infty} ; \\
& \operatorname{Ri}_{i}=g \theta_{i} h_{i}^{\prime} / v_{i}{ }^{2} ; \\
& m_{i}^{*}=\dot{m}_{i} /\left(\rho_{\infty} g^{1 / 2} D^{5 / 2}\right) ; \\
& Q_{D 1}^{*}=\dot{Q} /\left(\rho_{\infty} C_{p} T_{\infty} g^{1 / 2} D^{5 / 2}\right) ; \\
& Q_{D 2}^{*}=\left(\frac{m_{p}^{*}}{n_{p}}+\frac{m_{j}^{*}}{n_{j}}\right) \lambda
\end{aligned}
$$




\section{Appendix B: Flame Height Prediction, $H_{f}$}

The free flame height, $H_{f}$, given in Figure 1 was obtained from the measurements of You and Faeth [2]. In general, a formula is needed for predicting a typical free flame height. We chose to use the model of Grove and Quintiere [1] tailored to the free flame height data of You. Combine Eqns. $(6 \& 7)$ with $\dot{m}_{j}=0$,

$Q_{D}^{*}=\left(\lambda C_{e} / n_{p}\right)(z / D)^{1 / 2}\left(1+2 C_{l} z / D\right)^{2}$

where $Q_{D}^{*}=\dot{Q} /\left(\rho_{\infty} c_{p} T_{\infty} g^{1 / 2} D^{5 / 2}\right)$

With substitution of values for constants in the equation, a good approximation can be made that $(z / D)^{1 / 2}\left(1+2 C_{l} z / D\right)^{2} \cong(0.832+0.455 z / D)^{5 / 2}, z / D \geq 0.5$

$(z / D)^{1 / 2}\left(1+2 C_{l} z / D\right)^{2} \cong(-0.013+1.33 z / D)^{5 / 2}, z / D<0.5$

With Eqns. (B-1 \& B-2), free flame height in terms of heat release rate is carried out for the data range of You as

$H_{f} / D=6.17 Q_{D}^{* 2 / 5}-1.83, Q_{D}^{*}=\dot{Q} /\left(\rho_{\infty} c_{p} T_{\infty} g^{1 / 2} D^{5 / 2}\right)$, for $Q_{D}^{*}>0.1$

Figure 10 shows the fit of the correlation. 


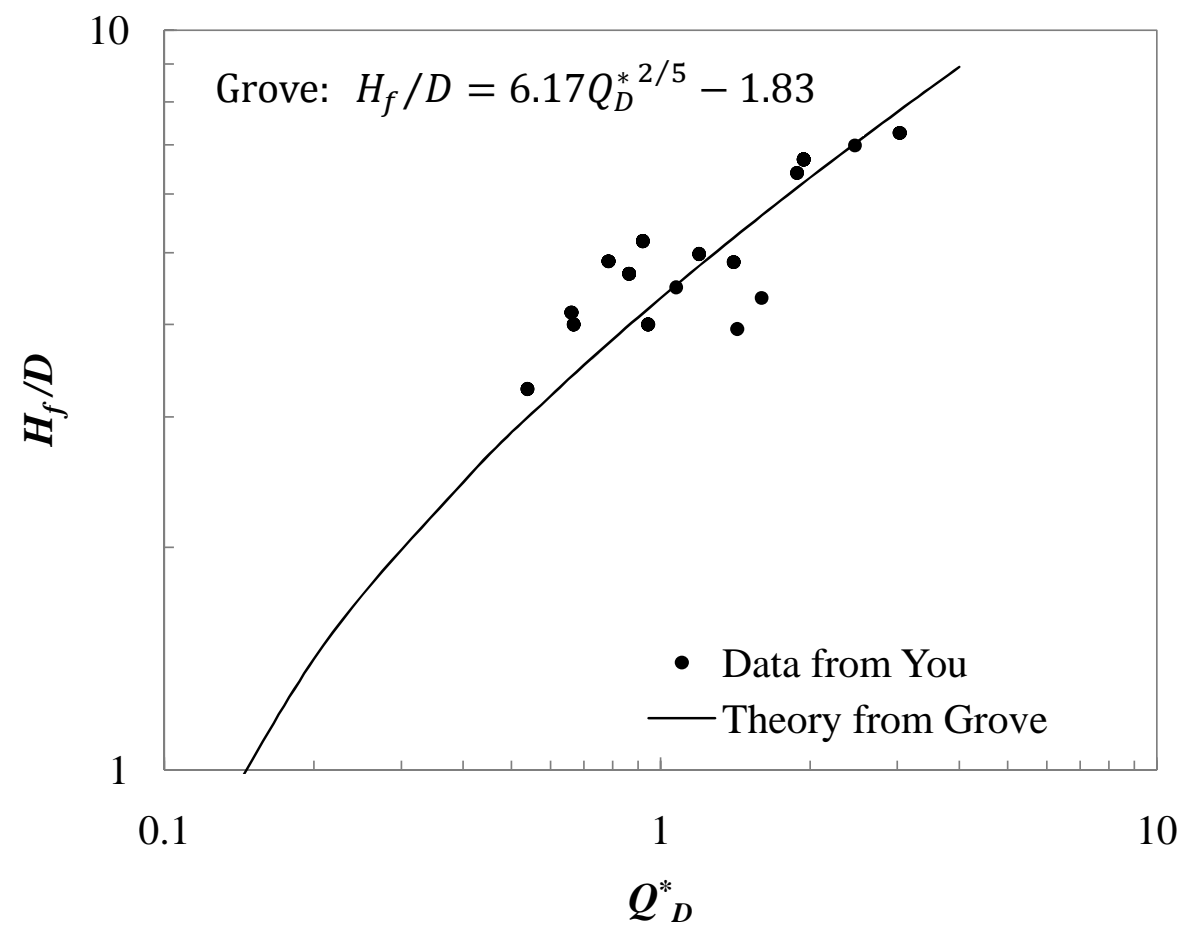

Figure 10. Free flame height prediction tailored to data of You [2].

Figure 11 shows the free flame height predictions from Grove and Heskestad compared to a wide range of data studied by previous investigators. 


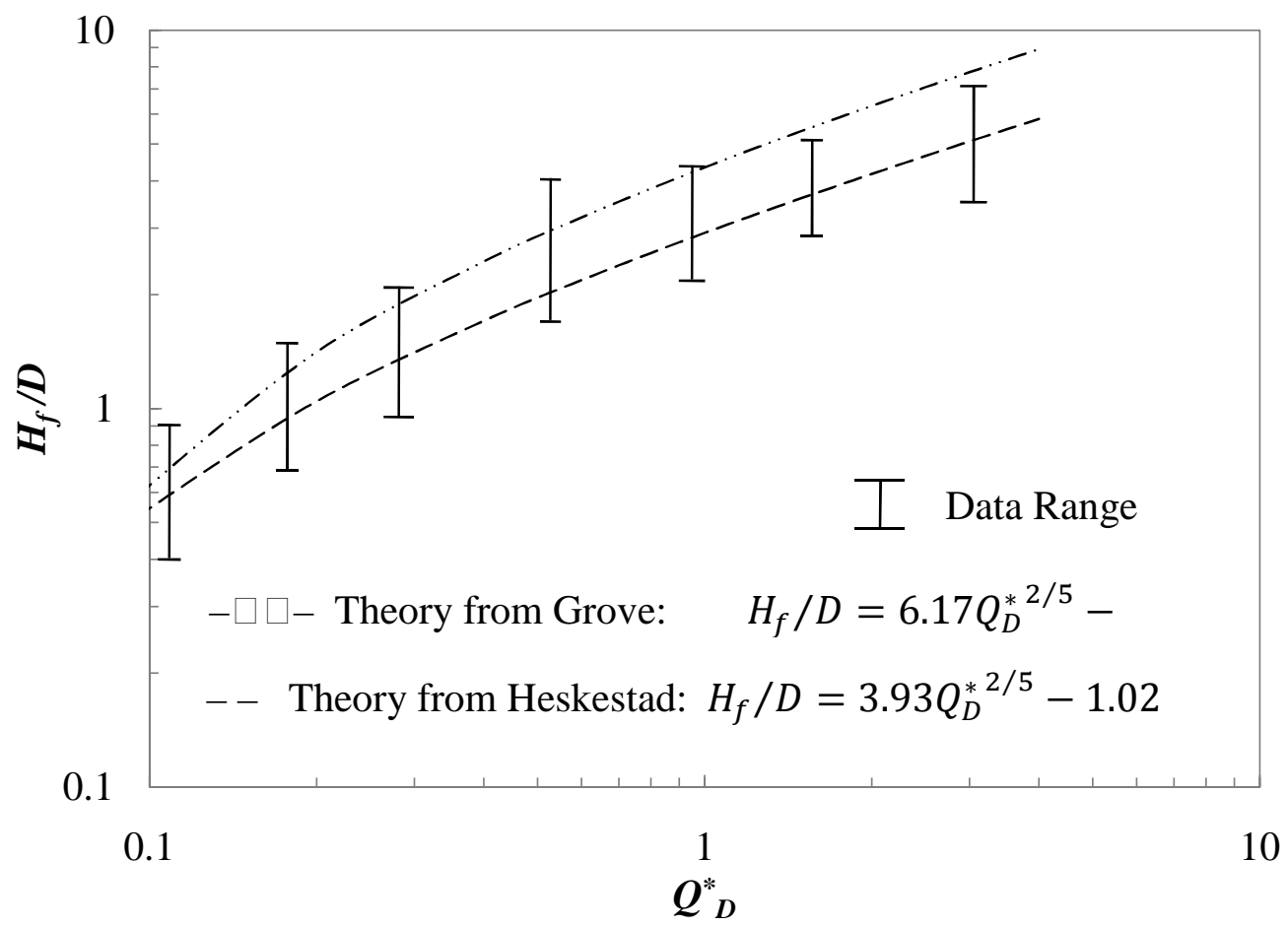

Figure 11. Free flame height predictions compared to a wide range of data. 


\section{Appendix C: Derivation and Formulation}

\section{C.1. Governing Equations}

Consider mass, momentum and energy conservations of steady flow to derive the governing equations for the ceiling jet. Assume Bousinnesq approximation in which density is constant, $\rho \approx \rho_{\infty}$, except in the buoyancy term. We start momentum conservation with the Navier-Stokes equations in cylindrical coordinates for turbulent-steady flow.

Mass conservation:

$\rho_{\infty}\left(\frac{1}{r} \frac{\partial(r v)}{\partial r}+\frac{\partial u}{\partial y}\right)=0$

Momentum conservation:

$y$-momentum:

$\rho_{\infty}\left(v \frac{\partial u}{\partial r}+u \frac{\partial u}{\partial y}\right)=\rho g-\frac{\partial p}{\partial y}+\mu\left[\frac{1}{r} \frac{\partial}{\partial r}\left(r \frac{\partial u}{\partial r}\right)+\frac{\partial^{2} u}{\partial y^{2}}\right]$

$r$-momentum:

$\rho_{\infty}\left(v \frac{\partial v}{\partial r}+u \frac{\partial v}{\partial y}\right)=-\frac{\partial p}{\partial r}+\mu\left[\frac{\partial}{\partial r}\left(\frac{1}{r} \frac{\partial(r v)}{\partial r}\right)+\frac{\partial^{2} v}{\partial y^{2}}\right]$

Energy conservation:

$\rho_{\infty} C_{p}\left(v \frac{\partial T}{\partial r}+u \frac{\partial T}{\partial y}\right)=k\left[\frac{1}{r} \frac{\partial}{\partial r}\left(r \frac{\partial T}{\partial r}\right)+\frac{\partial^{2} T}{\partial y^{2}}\right]+\dot{q}^{\prime \prime \prime}$

where $k$ is the thermal conductivity.

We simplify governing equations by following processes.

Mass conservation:

$\frac{1}{r} \frac{\partial(r v)}{\partial r}+\frac{\partial u}{\partial y}=0$

Momentum conservation: 
$y$-momentum:

To simplify the momentum equations, we shall apply boundary layer character to the ceiling jet where $\frac{h_{e}}{H}$ is small (of the order of 0.1), and the Reynolds number is large, $\frac{1}{R e} \sim\left(\frac{h_{e}}{H}\right)^{2}$.

We now rewrite the Navier-Stokes equations in dimensionless form to estimate the order of magnitude of each term in order to be able to drop small terms and thus to achieve the desired simplification of the equations.

Define

$\rho^{\prime} \equiv \frac{\rho}{\rho_{\infty}}, p^{\prime} \equiv \frac{p}{\rho_{\infty} v_{e}^{2}}, r^{\prime} \equiv \frac{r}{H}, y^{\prime} \equiv \frac{y}{h_{e}}, v^{\prime} \equiv \frac{v}{v_{e}}, u^{\prime} \equiv \frac{u}{v_{e} \frac{h_{e}}{H}}$

and Reynolds number

$R e \equiv \frac{\rho_{\infty} v_{e} H}{\mu}$.

Substitute to Eq. (C-2) and multiply both sides by $\frac{h_{e}}{H}$ yields $y$-momentum in dimensionless form:

$\left(\frac{h_{e}}{H}\right)^{2}\left(v^{\prime} \frac{\partial u^{\prime}}{\partial r^{\prime}}+u^{\prime} \frac{\partial u \prime}{\partial y^{\prime}}\right)=\frac{g H}{v_{e}^{2}} \frac{h_{e}}{H} \rho^{\prime}-\frac{\partial p^{\prime}}{\partial y^{\prime}}+\frac{1}{R e}\left(\frac{h_{e}}{H}\right)^{2} \frac{1}{r \prime} \frac{\partial}{\partial r^{\prime}}\left(r^{\prime} \frac{\partial u^{\prime}}{\partial r^{\prime}}\right)+\frac{1}{R e} \frac{\partial^{2} u \prime}{\partial y^{\prime 2}}$

Note that both $\frac{h_{e}}{H}$ and $\frac{1}{R e}$ are small. It allows us to drop small terms and the remaining equation becomes

$\frac{g H}{v_{e}^{2}} \frac{h_{e}}{H} \rho^{\prime}-\frac{\partial p^{\prime}}{\partial y^{\prime}} \approx 0$

$v_{e}$ is the vertical flow velocity in the plume. This velocity behaves different for the far-field and near-field models.

In Alpert's model, which is the far-field, $\frac{g H}{v_{e}^{2}} \frac{h_{e}}{H} \sim 1$, 
$\frac{\partial p}{\partial y} \approx \rho g$.

In our model, which is the near-field,

$v_{e}=C_{v} \sqrt{g H}$

where $C_{v}=2.02$ and $\frac{h_{e}}{H} \sim 0.1$, thus

$\frac{g H}{v_{e}^{2}} \frac{h_{e}}{H} \sim 0.025$

It essentially yields that the pressure across the jet is constant,

$\frac{\partial p}{\partial y} \approx 0$

Integral over $0 \leq y \leq h$ yeilds

$\int_{y}^{h} \frac{\partial p}{\partial y} d y=p(h, r)-p(y, r) \approx 0$.

At $y=h$,

$p=p_{a}$

where $p_{a}$ is the pressure at atmosphere, so

$p(y, r) \approx p_{a}$.

It is saying that the entrainment vertically upward into the ceiling jet is not due to pressure difference, but turbulence and friction. The pressure difference term disappeared in our near-field model, while it was kept in the far-field model by Alpert [6] for point source plume with a different $v_{e}$.

$r$-momentum:

Applying similar process to Eq. (C-3) leads to the dimensionless form 
$v^{\prime} \frac{\partial v^{\prime}}{\partial r^{\prime}}+u^{\prime} \frac{\partial v^{\prime}}{\partial y^{\prime}}=-\frac{\partial p^{\prime}}{\partial r^{\prime}}+\frac{1}{R_{e}} \frac{\partial}{\partial r^{\prime}}\left(\frac{1}{r^{\prime}} \frac{\partial r^{\prime} v^{\prime}}{\partial r^{\prime}}\right)+\frac{1}{R_{e}}\left(\frac{H}{h_{e}}\right)^{2} \frac{\partial^{2} v^{\prime}}{\partial y^{\prime}}$.

It simplifies $r$-momentum equation as

$\rho_{\infty}\left(v \frac{\partial v}{\partial r}+u \frac{\partial v}{\partial y}\right)=-\frac{\partial p}{\partial r}+\mu \frac{\partial^{2} v}{\partial y^{2}}$

From Eq. (C-13), where $p_{a}$ is a function of $y$ direction only

$\frac{\partial p}{\partial r}=\frac{\partial p(y, r)}{\partial r} \approx \frac{\partial p_{a}}{\partial r}=0$

$r$-momentum equation becomes

$\rho_{\infty}\left(v \frac{\partial v}{\partial r}+u \frac{\partial v}{\partial y}\right)=\mu \frac{\partial^{2} v}{\partial y^{2}}$

Energy conservation:

Now consider the energy equation, Eq. (C-4). Ceiling jet is considered as a very thin boundary layer, thus the temperature change along the $r$ direction can be neglected compared to that along the $y$ direction,

$$
k \frac{1}{r} \frac{\partial}{\partial r}\left(r \frac{\partial T}{\partial r}\right) \approx 0
$$

Replace $T$ with $T-T_{\infty}$. It yields

$\rho_{\infty} c_{p}\left[v \frac{\partial\left(T-T_{\infty}\right)}{\partial r}+u \frac{\partial\left(T-T_{\infty}\right)}{\partial y}\right]=k \frac{\partial^{2}\left(T-T_{\infty}\right)}{\partial y^{2}}+\dot{q}^{\prime \prime \prime}$

With the preceding assumptions, the governing equations become

Mass: $\quad \frac{1}{r} \frac{\partial(r v)}{\partial r}+\frac{\partial u}{\partial y}=0$

Momentum: $\quad \rho_{\infty}\left(v \frac{\partial v}{\partial r}+u \frac{\partial v}{\partial y}\right)=\mu \frac{\partial^{2} v}{\partial y^{2}}$

Energy: $\quad \rho_{\infty} c_{p}\left[v \frac{\partial\left(T-T_{\infty}\right)}{\partial r}+u \frac{\partial\left(T-T_{\infty}\right)}{\partial y}\right]=k \frac{\partial^{2}\left(T-T_{\infty}\right)}{\partial y^{2}}+\dot{q}^{\prime \prime \prime}$ 


\section{C.2. Integral Governing Equations over $0 \leq y \leq h$}

Integration of the governing equations over $0 \leq y \leq h$ is carried out, following Alpert [6] and You $[7,8]$ using top-hat profiles, i.e. uniform quantities along the $y$ direction.

Mass:

$\int_{0}^{h}\left(\frac{1}{r} \frac{\partial(r v)}{\partial r}+\frac{\partial u}{\partial y}\right) d y=0$

Introduce the entrainment velocity, $u_{e n t}$, to the turbulent model,

$[u]_{y=h}=u_{e n t}$.

$\int_{0}^{h}\left(\frac{1}{r} \frac{\partial(r v)}{\partial r}+\frac{\partial u}{\partial y}\right) d y=\frac{1}{r} \frac{d}{d r}(r v) \int_{0}^{h} d y+\int_{0}^{h} \frac{\partial u}{\partial y} d y$

$$
\begin{aligned}
& =\frac{1}{r} \frac{d}{d r}(r v) h-[u]_{y=0}^{y=h} \\
& =\frac{1}{r} \frac{d}{d r}(r v h)-u_{e n t}
\end{aligned}
$$

Note $u_{e n t}=E v$ from Chapter 2.6. It yields

$$
\frac{d}{d r}(r v h)=E r v .
$$

Momentum:

$$
\begin{aligned}
\text { Left } & =\int_{0}^{h} \rho_{\infty}\left(v \frac{\partial v}{\partial r}+u \frac{\partial v}{\partial y}\right) d y \\
& =\int_{0}^{h} \frac{\rho_{\infty}}{r}\left(r v \frac{\partial v}{\partial r}+r u \frac{\partial v}{\partial y}\right) d y \\
& =\frac{\rho_{\infty}}{r} \int_{0}^{h}\left(\frac{\partial\left(r v^{2}\right)}{\partial r}-v \frac{\partial(r v)}{\partial r}+\frac{\partial(r u v)}{\partial y}-v \frac{\partial(r u)}{\partial y}\right) d y
\end{aligned}
$$

Multiply Eq. (C-22) by $v r$,

$v \frac{\partial(r v)}{\partial r}+v \frac{\partial(r u)}{\partial y}=0$.

Realize that 
$\frac{d}{d t} \int_{x=a(t)}^{x=b(t)} f(x, t) d x=\int_{a}^{b} \frac{\partial f(x, t)}{\partial t} d x+f(b, t) \frac{d b}{d t}-f(a, t) \frac{d a}{d t}$.

Now

$$
\begin{aligned}
\text { Left } & =\frac{\rho_{\infty}}{r} \int_{0}^{h}\left(\frac{\partial\left(r v^{2}\right)}{\partial r}+\frac{\partial(r u v)}{\partial y}\right) d y \\
& =\frac{\rho_{\infty}}{r} \frac{d}{d r}\left(r v^{2} h\right) .
\end{aligned}
$$

$$
\begin{aligned}
\text { Right } & =\int_{0}^{h} \mu \frac{\partial^{2} v}{\partial y^{2}} d y \\
& =\mu \int_{0}^{h} \frac{\partial}{\partial y}\left(\frac{\partial v}{\partial y}\right) d y \\
& =\mu\left[\frac{\partial v}{\partial y}\right]_{y=0}^{y=h} \\
& =0-\mu\left[\frac{\partial v}{\partial y}\right]_{y=0} .
\end{aligned}
$$

Note that

$$
\tau_{w}=\mu\left[\frac{\partial v}{\partial y}\right]_{y=0}
$$

and from Alpert (6)

$\tau_{w}=\frac{f}{2} \rho_{\infty} v^{2}$

where $f$ is the friction factor.

The momentum equation becomes

$\frac{d}{d r}\left(r v^{2} h\right)=-f r v^{2} / 2$.

Energy:

$\int_{0}^{h} \rho_{\infty} c_{p}\left(v \frac{\partial\left(T-T_{\infty}\right)}{\partial r}+u \frac{\partial\left(T-T_{\infty}\right)}{\partial y}\right) d y=\int_{0}^{h}\left(k \frac{\partial^{2}\left(T-T_{\infty}\right)}{\partial y^{2}}+\dot{q}^{\prime \prime \prime}\right) d y$ 
Multiply equation by $r / r$,

$$
\begin{aligned}
& \frac{1}{r} \int_{0}^{h} \rho_{\infty} c_{p}\left(r v \frac{\partial\left(T-T_{\infty}\right)}{\partial r}+r u \frac{\partial\left(T-T_{\infty}\right)}{\partial y}\right) d y \\
& =\frac{\rho_{\infty} c_{p}}{r} \int_{0}^{h}\left(\frac{\partial r v\left(T-T_{\infty}\right)}{\partial r}-\left(T-T_{\infty}\right) \frac{\partial r v}{\partial r}+\frac{\partial r u\left(T-T_{\infty}\right)}{\partial y}-\left(T-T_{\infty}\right) \frac{\partial r u}{\partial y}\right) d y
\end{aligned}
$$

Apply similar methods as we derived the $r$-momentum equation. Apply Eq. (C-30) then

$$
\left(T-T_{\infty}\right) \frac{\partial r v}{\partial r}+\left(T-T_{\infty}\right) \frac{\partial r u}{\partial y}=0 .
$$

Left $=\frac{\rho_{\infty} c_{p}}{r} \int_{0}^{h}\left(\frac{\partial r v\left(T-T_{\infty}\right)}{\partial r}+\frac{\partial r u\left(T-T_{\infty}\right)}{\partial y}\right) d y$.

With top-hat profile, i.e. uniform properties along $y$-direction, apply the same mathematical conversion as Eq. (C-31),

$$
\begin{aligned}
& \begin{aligned}
\text { Left } & =\frac{\rho_{\infty} c_{p}}{r}\left(\int_{0}^{h} \frac{d r v\left(T-T_{\infty}\right)}{d r} d y+\int_{0}^{h} \frac{d r u\left(T-T_{\infty}\right)}{d y} d y\right) \\
& =\frac{\rho_{\infty} c_{p}}{r}\left(\frac{d}{d r} \int_{0}^{h} r v\left(T-T_{\infty}\right) d y+\left[r u\left(T-T_{\infty}\right)\right]_{y=0}^{y=h}\right) \\
& =\frac{\rho_{\infty} c_{p}}{r} \frac{d}{d r}\left(r v h\left(T-T_{\infty}\right)\right)
\end{aligned} \\
& \text { Right }=\int_{0}^{h} k \frac{\partial^{2}\left(T-T_{\infty}\right)}{\partial y^{2}} d y+\int_{0}^{h} \dot{q}^{\prime \prime \prime} d y \\
& \int_{0}^{h} k \frac{\partial^{2}\left(T-T_{\infty}\right)}{\partial y^{2}} d y=\left[k \frac{\partial\left(T-T_{\infty}\right)}{\partial y}\right]_{y=0}^{y=h}=0-k\left[\frac{\partial T}{\partial y}\right]_{y=0} \\
& \dot{q}_{w}^{\prime \prime} \equiv k\left[\frac{\partial T}{\partial y}\right]_{y=0} \text { is the convective heat loss from the flame to the ceiling, } \\
& \dot{q}_{w}^{\prime \prime}=h_{c}\left(T-T_{w}\right)
\end{aligned}
$$

where $h_{c}$ is the convective heat transfer coefficient and $T_{w}$ is the ceiling temperature.

Assume adiabatic in the ceiling jet flame region after $r>r_{e}$, i.e. no heat loss to the ceiling. Therefore 
$\dot{q}_{w}^{\prime \prime}=0$

and right side of the energy equation becomes

Right $=\int_{0}^{h} \dot{q}^{\prime \prime \prime} d y$.

$\dot{q}^{\prime \prime \prime}$ is the heat generated by combustion, which depends on the air entrainment. The rate of combustion energy in the ceiling jet region is expressed as

$\dot{q}=\dot{q}^{\prime \prime \prime} 2 \pi r h d r$

It can also be expressed in terms of the amount of air burned

$$
\begin{aligned}
\dot{q} & =d \dot{m}_{j} \cdot\left(\frac{\Delta h_{c}}{n_{j} s}\right) \\
& =\rho_{\infty} u_{e n t} 2 \pi r d r\left(\frac{\Delta h_{c}}{n_{j} s}\right)
\end{aligned}
$$

Combining above two equations yields

$\dot{q}^{\prime \prime \prime}=\rho_{\infty} \frac{u_{e n t}}{h} \frac{\Delta h_{c}}{n_{j} s}$

Note $u_{e n t}=E v$ as stated previously,

$\dot{q}^{\prime \prime \prime}=\rho_{\infty} \frac{E v}{h} \frac{\Delta h_{c}}{n_{j} s}$

and thus

$$
\begin{aligned}
\text { Right } & =\dot{q}^{\prime \prime \prime} h \\
& =\rho_{\infty} E v \frac{\Delta h_{c}}{n_{j} s}
\end{aligned}
$$

Thus the conservation of energy becomes

$\frac{d}{d r}\left(r v h\left(T-T_{\infty}\right)\right)=\operatorname{Erv} \frac{\Delta h_{c}}{s c_{p} n_{j}}$ 
With $\theta \equiv\left(T-T_{\infty}\right) / T_{\infty}$ and $\equiv \frac{\Delta h_{c}}{s C_{p} T_{\infty}}$, it yields

$\frac{d}{d r}(r v h \theta)=\operatorname{Erv} \lambda / n_{j}$

The following ordinary differential equations result for the axisymmetric ceiling jet flame:

Mass: $\quad \frac{d}{d r}(r v h)=E r v$

Momentum: $\quad \frac{d}{d r}\left(r v^{2} h\right)=-f r v^{2} / 2$

Energy: $\quad \frac{d}{d r}(r v h \theta)=\operatorname{Erv} \lambda / n_{j}$

where $E$ is the entrainment coefficient and $f$ the friction factor.

C.3. Integral Governing Equations over $r_{e} \leq r \leq r_{f}$

$h_{f}:$

Assume $E$ is constant. Eq. (C-54) $\times v-$ Eq. (C-55) yields

$\frac{h}{v} \frac{d v}{d r}=-\left(E+\frac{f}{2}\right)$

Rewrite Eq. (C-54) as

$v h+r\left(h \frac{d v}{d r}+v \frac{d h}{d r}\right)=E r v$

Subtracting Eq. (C-57) yields

$\frac{d(r h)}{d r}=\left(2 E+\frac{f}{2}\right) r$

Assume $E$ and $f$ are constant at this point. Integrating it over $r_{e} \leq r \leq r_{f}$ yields

$h_{f}=(E+f / 4) r_{f}-\left(r_{e} / r_{f}\right)\left[(E+f / 4) r_{e}-h_{e}\right]$ 
$v_{f}$ :

Rewrite Eq. (C-57)

$\frac{d v}{v}=-\left(E+\frac{f}{2}\right) \frac{d r}{h}$

Integrate it over $r_{e} \leq r \leq r_{f}$

$\frac{v_{f}}{v_{e}}=\exp \left(-\int_{r_{e}}^{r_{f}}\left(\frac{E+\frac{f}{2}}{h}\right) d r\right)$

Substitute $h=h_{f}$, i.e. Eq. (C-60)

$v_{f}=C_{v}(g H)^{1 / 2}\left[(E+f / 4)\left(r_{e}{ }^{2} / r_{f} h_{f}-r_{f} / h_{f}\right)+1\right]^{(E+f / 2) /(2 E+f / 2)}$

$\theta_{f}:$

Substitute Eq. (C-54) to Eq. (C-56)

$\frac{d}{d r}(r v h \theta)=\frac{\lambda}{n_{j}} \frac{d}{d r}(r v h)$

Integrate it over $r_{e} \leq r \leq r_{f}$

$\theta_{f}=\left(\left(r_{f} v_{f} h_{f}-r_{e} v_{e} h_{e}\right)\left(\lambda / n_{j}\right)+r_{e} v_{e} h_{e} \theta_{e}\right) /\left(r_{f} v_{f} h_{f}\right)$ 


\section{Appendix D: Calculations for Flame Length, $r_{f}$ and Error Examination}

The results for flame length vs. fire power are shown in Figure 3. The markers represent data extracted from Table 2, with $x$-axis $Q_{D}^{*}$ from column $Q_{D_{1}}^{*}$ and $y$-axis $r_{f} / H$ from column $r_{f}$. The solid lines represent the calculation results based on theory, extracted from Table 3 below, with $x$-axis $Q_{D}^{*}$ from column $Q_{D_{3}}^{*}$ and $y$-axis $r_{f} / H$ from column $r_{f}$. Data in Table 3 is grouped by $D / H$ value in a decrease order. The $D / H$ value for each data set is shown in Figure 4 as well as its legend. The dash line marks the starting position of flame length for each fixed $D / H$. It represents the condition where $Q_{D}^{*}$ is barely large enough for the flame to reach the ceiling, and $r_{f}{ }^{\prime}=r_{e}{ }^{\prime}$. Those values are also extracted from Table 4 , with $x$-axis $Q_{D}^{*}$ from column $Q_{D_{3}}^{*}$ and $y$-axis $r_{f} / H$ from column $r_{e}$ ’.

The purpose of Table 3 is to calculate values for $r_{f}^{\prime}$ vs. $Q_{D}^{*}$ using Eq. (37 \& 38). As stated before, because of the nature of the format of Eq. (37), it is easier to assume $r_{f}^{\prime}$ as an input value to solve $Q_{D}^{*}$, than the other way around. $D$ and $H$ are selected from Table 2 in order to compare theory with data. $r_{f}{ }^{\prime}$ value is selected as a multiple of $r_{e}{ }^{\prime}$.

Taking $r_{f}^{\prime}$ as a known parameter to solve $Q_{D}^{*}$, values for other parameters are needed in order to use Eq. (37), such as $h_{f}, v_{f}, \theta_{f}$ and $n_{j}$. Derivations for those parameters are presented in Appendix C. Equations are listed below:

$$
\begin{aligned}
& h_{f}=(E+f / 4) r_{f}-\left(r_{e} / r_{f}\right)\left[(E+f / 4) r_{e}-h_{e}\right] \\
& v_{f}=C_{v}(g H)^{1 / 2}\left[(E+f / 4)\left(r_{e}^{2} / r_{f} h_{f}-r_{f} / h_{f}\right)+1\right]^{(E+f / 2) /(2 E+f / 2)} \\
& \theta_{f}=\left(\left(r_{f} v_{f} h_{f}-r_{e} v_{e} h_{e}\right)\left(\lambda / n_{j}\right)+r_{e} v_{e} h_{e} \theta_{e}\right) /\left(r_{f} v_{f} h_{f}\right)
\end{aligned}
$$


$\mathrm{Ri}_{f}=g \theta_{f} v_{f}^{2}$

$n_{j}=9.6 \exp \left[-\left(2.26\left(\mathrm{R}_{\mathrm{i}_{f}}-\mathrm{R}_{\mathrm{i}_{e}}+0.05\right) /(0.197-0.091 D / H)\right)\right]$

$E_{f}=\left(0.075 /\left(\theta_{f}+1\right)^{1 / 2}\right) e^{-5 \mathrm{Ri}_{f}}$

Iterations were involved again to solve the six variables with the six equations. We discovered multiple solutions in some of the cases. Of those solutions, we selected the one that is most physically reasonable (e.g., $r_{f}$ increases as $\dot{Q}$ increases). In Table 3 , columns $h_{f}{ }^{\prime}$ to $E_{f}$ show the full results. $Q_{D_{3}}^{*}$ is then computed using Eq. (37), i.e.

$\dot{Q}=\left(\rho_{\infty} \Delta h_{c} / s\right)\left[g^{1 / 2} D^{5 / 2} C_{e}(z / D)^{1 / 2}\left(1+2 C_{l} z / D\right)^{2} / 9.6+2 \pi\left(r_{f} v_{f} h_{f}-r_{e} v_{e} h_{e}\right) / n_{j}\right]$

$z=H-h_{e}$

or equivalently,

$\dot{Q}=\left(\frac{\dot{m}_{p}}{n_{p}}+\frac{\dot{m}_{j}}{n_{j}}\right)\left(\frac{\Delta h_{c}}{s}\right)$

Table 4 examines theory vs. data agreements for flame length results. Experimental data, $Q_{D_{1}}^{*}$ and $r_{f}^{\prime}$, are both taken from Table 2 . Theoretical prediction is extracted from the theory lines in Figure 4 so that the heat release rate of the extracted data, $Q_{D_{4}}^{*}$, has the same value as $Q_{D_{1}}^{*}$. Because direct computation of $r_{f}^{\prime}$ from $Q_{D}^{*}$ met mathematical difficulties, the extraction of data is done by using a graph digitizer software, and as a result slight errors may apply $\left(Q_{D_{4}}^{*} \cong Q_{D_{1}}^{*}\right)$.

The relative error from theory to data is calculated as

Error $=\left(r_{f}^{\prime}\right.$, theory $-r_{f}^{\prime}$, data $) / r_{f}^{\prime}$, data

The average error above data is computed to be $23.40 \%$ and the average error below data is $10.31 \%$, shown at the bottom of the table. 
Table 3. Calculations for flame length, $r_{f}$.

\begin{tabular}{|c|c|c|c|c|c|c|c|c|c|c|c|c|c|c|c|c|c|}
\hline$D(\mathrm{~m})$ & $H(\mathrm{~m})$ & $D / H$ & $r_{e}{ }^{\prime}$ & $v_{e}^{\prime}$ & $h_{e}{ }^{\prime}$ & $\theta_{e}$ & $r_{f}^{\prime}$ & $r_{f}^{\prime} / r_{e}{ }^{\prime}$ & $h_{f}^{\prime}$ & $v_{f}^{\prime}$ & $\theta_{f}$ & $R i_{f}$ & $n j$ & $E_{f}$ & $m_{p}^{*}$ & $m_{j}^{*}$ & $Q_{D_{3}}^{*}$ \\
\hline 0.064 & 0.015 & 4.27 & 2.31 & 2.02 & 0.90 & 1.91 & 2.31 & 1 & 0.90 & 2.02 & 1.89 & 0.42 & 1.00 & 0.005 & 0.01 & 0.00 & 0.01 \\
\hline 0.064 & 0.015 & 4.27 & 2.31 & 2.02 & 0.90 & 1.91 & 2.77 & 1.2 & 0.76 & 2.00 & 1.92 & 0.37 & 1.00 & 0.007 & 0.01 & 0.00 & 0.04 \\
\hline 0.064 & 0.015 & 4.27 & 2.31 & 2.02 & 0.90 & 1.91 & 3.24 & 1.4 & 0.66 & 1.98 & 1.97 & 0.34 & 1.00 & 0.008 & 0.01 & 0.01 & 0.08 \\
\hline 0.064 & 0.015 & 4.27 & 2.31 & 2.02 & 0.90 & 1.91 & 3.70 & 1.6 & 0.59 & 1.95 & 2.03 & 0.32 & 1.00 & 0.009 & 0.01 & 0.01 & 0.13 \\
\hline 0.064 & 0.015 & 4.27 & 2.31 & 2.02 & 0.90 & 1.91 & 4.16 & 1.8 & 0.54 & 1.92 & 2.09 & 0.31 & 1.00 & 0.009 & 0.01 & 0.02 & 0.19 \\
\hline 0.064 & 0.015 & 4.27 & 2.31 & 2.02 & 0.90 & 1.91 & 4.62 & 2 & 0.50 & 1.88 & 2.16 & 0.30 & 1.00 & 0.009 & 0.01 & 0.02 & 0.25 \\
\hline 0.064 & 0.015 & 4.27 & 2.31 & 2.02 & 0.90 & 1.91 & 5.09 & 2.2 & 0.47 & 1.85 & 2.22 & 0.30 & 1.00 & 0.009 & 0.01 & 0.03 & 0.31 \\
\hline 0.064 & 0.015 & 4.27 & 2.31 & 2.02 & 0.90 & 1.91 & 5.55 & 2.4 & 0.44 & 1.82 & 2.29 & 0.31 & 1.00 & 0.009 & 0.01 & 0.04 & 0.38 \\
\hline 0.064 & 0.015 & 4.27 & 2.31 & 2.02 & 0.90 & 1.91 & 6.01 & 2.6 & 0.42 & 1.78 & 2.35 & 0.31 & 1.00 & 0.009 & 0.01 & 0.04 & 0.44 \\
\hline 0.064 & 0.015 & 4.27 & 2.31 & 2.02 & 0.90 & 1.91 & 6.47 & 2.8 & 0.40 & 1.75 & 2.41 & 0.31 & 1.00 & 0.008 & 0.01 & 0.05 & 0.49 \\
\hline 0.064 & 0.015 & 4.27 & 2.31 & 2.02 & 0.90 & 1.91 & 6.94 & 3 & 0.38 & 1.71 & 2.46 & 0.32 & 1.00 & 0.008 & 0.01 & 0.05 & 0.55 \\
\hline 0.064 & 0.015 & 4.27 & 2.31 & 2.02 & 0.90 & 1.91 & 9.25 & 4 & 0.41 & 1.40 & 3.51 & 0.73 & 1.00 & 0.001 & 0.01 & 0.18 & 1.77 \\
\hline 0.064 & 0.015 & 4.27 & 2.31 & 2.02 & 0.90 & 1.91 & 11.56 & 5 & 0.43 & 1.19 & 4.17 & 1.25 & 1.00 & 0.000 & 0.01 & 0.28 & 2.78 \\
\hline 0.064 & 0.015 & 4.27 & 2.31 & 2.02 & 0.90 & 1.91 & 13.87 & 6 & 0.42 & 1.06 & 4.50 & 1.70 & 1.00 & 0.000 & 0.01 & 0.34 & 3.37 \\
\hline 0.064 & 0.015 & 4.27 & 2.31 & 2.02 & 0.90 & 1.91 & 16.19 & 7 & 0.41 & 0.96 & 4.61 & 2.02 & 1.00 & 0.000 & 0.01 & 0.36 & 3.58 \\
\hline 0.064 & 0.02 & 3.20 & 1.78 & 2.02 & 0.69 & 1.91 & 1.78 & 1 & 0.69 & 2.02 & 1.89 & 0.32 & 1.00 & 0.009 & 0.02 & 0.00 & 0.03 \\
\hline 0.064 & 0.02 & 3.20 & 1.78 & 2.02 & 0.69 & 1.91 & 2.13 & 1.2 & 0.59 & 2.00 & 1.94 & 0.29 & 1.00 & 0.010 & 0.02 & 0.01 & 0.08 \\
\hline 0.064 & 0.02 & 3.20 & 1.78 & 2.02 & 0.69 & 1.91 & 2.49 & 1.4 & 0.52 & 1.97 & 2.00 & 0.27 & 1.00 & 0.011 & 0.02 & 0.01 & 0.14 \\
\hline 0.064 & 0.02 & 3.20 & 1.78 & 2.02 & 0.69 & 1.91 & 2.85 & 1.6 & 0.46 & 1.94 & 2.07 & 0.26 & 1.00 & 0.012 & 0.02 & 0.02 & 0.22 \\
\hline 0.064 & 0.02 & 3.20 & 1.78 & 2.02 & 0.69 & 1.91 & 3.20 & 1.8 & 0.42 & 1.90 & 2.15 & 0.25 & 1.00 & 0.012 & 0.02 & 0.03 & 0.31 \\
\hline 0.064 & 0.02 & 3.20 & 1.78 & 2.02 & 0.69 & 1.91 & 3.56 & 2 & 0.39 & 1.87 & 2.23 & 0.25 & 1.00 & 0.012 & 0.02 & 0.04 & 0.40 \\
\hline 0.064 & 0.02 & 3.20 & 1.78 & 2.02 & 0.69 & 1.91 & 3.91 & 2.2 & 0.37 & 1.83 & 2.31 & 0.25 & 1.00 & 0.012 & 0.02 & 0.05 & 0.49 \\
\hline 0.064 & 0.02 & 3.20 & 1.78 & 2.02 & 0.69 & 1.91 & 5.34 & 3 & 0.30 & 1.69 & 2.58 & 0.27 & 1.00 & 0.010 & 0.02 & 0.08 & 0.83 \\
\hline 0.064 & 0.02 & 3.20 & 1.78 & 2.02 & 0.69 & 1.91 & 7.12 & 4 & 0.34 & 1.35 & 3.78 & 0.70 & 1.00 & 0.001 & 0.02 & 0.26 & 2.64 \\
\hline 0.064 & 0.02 & 3.20 & 1.78 & 2.02 & 0.69 & 1.91 & 8.90 & 5 & 0.36 & 1.14 & 4.50 & 1.26 & 1.00 & 0.000 & 0.02 & 0.41 & 4.09 \\
\hline 0.064 & 0.02 & 3.20 & 1.78 & 2.02 & 0.69 & 1.91 & 10.67 & 6 & 0.37 & 1.00 & 4.89 & 1.80 & 1.00 & 0.000 & 0.02 & 0.50 & 5.05 \\
\hline 0.064 & 0.02 & 3.20 & 1.78 & 2.02 & 0.69 & 1.91 & 12.45 & 7 & 0.36 & 0.91 & 5.08 & 2.25 & 1.00 & 0.000 & 0.02 & 0.56 & 5.58 \\
\hline 0.107 & 0.076 & 1.41 & 0.88 & 2.02 & 0.34 & 1.91 & 0.88 & 1 & 0.34 & 2.02 & 1.90 & 0.16 & 1.90 & 0.020 & 0.07 & 0.00 & 0.07 \\
\hline
\end{tabular}




\begin{tabular}{|c|c|c|c|c|c|c|c|c|c|c|c|c|c|c|c|c|c|}
\hline 0.107 & 0.076 & 1.41 & 0.88 & 2.02 & 0.34 & 1.91 & 1.06 & 1.2 & 0.30 & 1.99 & 1.92 & 0.14 & 3.28 & 0.021 & 0.07 & 0.02 & 0.13 \\
\hline 0.107 & 0.076 & 1.41 & 0.88 & 2.02 & 0.34 & 1.91 & 1.24 & 1.4 & 0.26 & 1.94 & 1.92 & 0.13 & 4.68 & 0.023 & 0.07 & 0.04 & 0.17 \\
\hline 0.107 & 0.076 & 1.41 & 0.88 & 2.02 & 0.34 & 1.91 & 1.41 & 1.6 & 0.24 & 1.90 & 1.90 & 0.13 & 5.80 & 0.023 & 0.07 & 0.07 & 0.20 \\
\hline 0.107 & 0.076 & 1.41 & 0.88 & 2.02 & 0.34 & 1.91 & 1.59 & 1.8 & 0.22 & 1.85 & 1.89 & 0.12 & 6.46 & 0.024 & 0.07 & 0.11 & 0.24 \\
\hline 0.107 & 0.076 & 1.41 & 0.88 & 2.02 & 0.34 & 1.91 & 1.77 & 2 & 0.21 & 1.80 & 1.88 & 0.12 & 6.64 & 0.024 & 0.07 & 0.14 & 0.28 \\
\hline 0.107 & 0.076 & 1.41 & 0.88 & 2.02 & 0.34 & 1.91 & 1.94 & 2.2 & 0.20 & 1.74 & 1.88 & 0.12 & 6.38 & 0.024 & 0.07 & 0.18 & 0.35 \\
\hline 0.107 & 0.076 & 1.41 & 0.88 & 2.02 & 0.34 & 1.91 & 2.12 & 2.4 & 0.19 & 1.70 & 1.89 & 0.13 & 5.77 & 0.023 & 0.07 & 0.21 & 0.44 \\
\hline 0.107 & 0.076 & 1.41 & 0.88 & 2.02 & 0.34 & 1.91 & 2.30 & 2.6 & 0.19 & 1.65 & 1.93 & 0.13 & 4.88 & 0.023 & 0.07 & 0.25 & 0.58 \\
\hline 0.107 & 0.076 & 1.41 & 0.88 & 2.02 & 0.34 & 1.91 & 2.47 & 2.8 & 0.18 & 1.61 & 2.02 & 0.14 & 3.76 & 0.021 & 0.07 & 0.27 & 0.80 \\
\hline 0.107 & 0.076 & 1.41 & 0.88 & 2.02 & 0.34 & 1.91 & 2.65 & 3 & 0.17 & 1.58 & 2.26 & 0.15 & 2.32 & 0.019 & 0.07 & 0.28 & 1.29 \\
\hline 0.107 & 0.076 & 1.41 & 0.88 & 2.02 & 0.34 & 1.91 & 2.83 & 3.2 & 0.16 & 1.59 & 2.97 & 0.19 & 1.00 & 0.015 & 0.07 & 0.25 & 2.60 \\
\hline 0.107 & 0.076 & 1.41 & 0.88 & 2.02 & 0.34 & 1.91 & 3.00 & 3.4 & 0.15 & 1.55 & 3.04 & 0.19 & 1.00 & 0.014 & 0.07 & 0.27 & 2.80 \\
\hline 0.107 & 0.076 & 1.41 & 0.88 & 2.02 & 0.34 & 1.91 & 3.18 & 3.6 & 0.15 & 1.52 & 3.11 & 0.20 & 1.00 & 0.013 & 0.07 & 0.29 & 2.98 \\
\hline 0.107 & 0.076 & 1.41 & 0.88 & 2.02 & 0.34 & 1.91 & 3.53 & 4 & 0.15 & 1.41 & 3.40 & 0.26 & 1.00 & 0.010 & 0.07 & 0.40 & 4.06 \\
\hline 0.107 & 0.076 & 1.41 & 0.88 & 2.02 & 0.34 & 1.91 & 4.41 & 5 & 0.17 & 1.17 & 2.33 & 0.29 & 1.00 & 0.021 & 0.07 & 0.70 & 7.03 \\
\hline 0.107 & 0.076 & 1.41 & 0.88 & 2.02 & 0.34 & 1.91 & 5.30 & 6 & 0.20 & 0.97 & 1.66 & 0.36 & 1.00 & 0.008 & 0.07 & 1.10 & 11.03 \\
\hline 0.107 & 0.076 & 1.41 & 0.88 & 2.02 & 0.34 & 1.91 & 6.18 & 7 & 0.23 & 0.83 & 1.55 & 0.51 & 1.00 & 0.004 & 0.07 & 1.44 & 14.44 \\
\hline 0.107 & 0.076 & 1.41 & 0.88 & 2.02 & 0.34 & 1.91 & 7.06 & 8 & 0.25 & 0.73 & 1.61 & 0.75 & 1.00 & 0.017 & 0.07 & 1.74 & 17.45 \\
\hline 0.064 & 0.05 & 1.28 & 0.82 & 2.02 & 0.32 & 1.91 & 1.31 & 1.6 & 0.22 & 1.89 & 1.91 & 0.12 & 5.66 & 0.024 & 0.08 & 0.08 & 0.23 \\
\hline 0.064 & 0.05 & 1.28 & 0.82 & 2.02 & 0.32 & 1.91 & 1.47 & 1.8 & 0.21 & 1.84 & 1.89 & 0.12 & 6.10 & 0.025 & 0.08 & 0.12 & 0.28 \\
\hline 0.064 & 0.05 & 1.28 & 0.82 & 2.02 & 0.32 & 1.91 & 1.64 & 2 & 0.20 & 1.79 & 1.89 & 0.12 & 6.17 & 0.025 & 0.08 & 0.16 & 0.34 \\
\hline 0.064 & 0.05 & 1.28 & 0.82 & 2.02 & 0.32 & 1.91 & 1.80 & 2.2 & 0.19 & 1.74 & 1.89 & 0.12 & 5.92 & 0.025 & 0.08 & 0.20 & 0.42 \\
\hline 0.064 & 0.05 & 1.28 & 0.82 & 2.02 & 0.32 & 1.91 & 1.97 & 2.4 & 0.18 & 1.69 & 1.90 & 0.12 & 5.41 & 0.024 & 0.08 & 0.24 & 0.52 \\
\hline 0.064 & 0.05 & 1.28 & 0.82 & 2.02 & 0.32 & 1.91 & 2.13 & 2.6 & 0.17 & 1.64 & 1.94 & 0.13 & 4.71 & 0.023 & 0.08 & 0.28 & 0.67 \\
\hline 0.064 & 0.05 & 1.28 & 0.82 & 2.02 & 0.32 & 1.91 & 2.29 & 2.8 & 0.17 & 1.60 & 2.01 & 0.13 & 3.87 & 0.022 & 0.08 & 0.31 & 0.88 \\
\hline 0.064 & 0.05 & 1.28 & 0.82 & 2.02 & 0.32 & 1.91 & 2.46 & 3 & 0.16 & 1.57 & 2.14 & 0.14 & 2.92 & 0.021 & 0.08 & 0.33 & 1.22 \\
\hline 0.064 & 0.05 & 1.28 & 0.82 & 2.02 & 0.32 & 1.91 & 2.62 & 3.2 & 0.15 & 1.55 & 2.50 & 0.16 & 1.75 & 0.018 & 0.08 & 0.33 & 1.97 \\
\hline 0.064 & 0.05 & 1.28 & 0.82 & 2.02 & 0.32 & 1.91 & 2.78 & 3.4 & 0.14 & 1.55 & 3.08 & 0.19 & 1.00 & 0.015 & 0.08 & 0.31 & 3.16 \\
\hline 0.064 & 0.05 & 1.28 & 0.82 & 2.02 & 0.32 & 1.91 & 2.95 & 3.6 & 0.14 & 1.51 & 3.15 & 0.19 & 1.00 & 0.014 & 0.08 & 0.33 & 3.37 \\
\hline 0.064 & 0.05 & 1.28 & 0.82 & 2.02 & 0.32 & 1.91 & 3.28 & 4 & 0.14 & 1.41 & 3.36 & 0.24 & 1.00 & 0.011 & 0.08 & 0.43 & 4.41 \\
\hline 0.064 & 0.05 & 1.28 & 0.82 & 2.02 & 0.32 & 1.91 & 4.10 & 5 & 0.16 & 1.17 & 2.43 & 0.28 & 1.00 & 0.010 & 0.08 & 0.76 & 7.69 \\
\hline
\end{tabular}




\begin{tabular}{|c|c|c|c|c|c|c|c|c|c|c|c|c|c|c|c|c|c|}
\hline 0.064 & 0.05 & 1.28 & 0.82 & 2.02 & 0.32 & 1.91 & 4.91 & 6 & 0.19 & 0.97 & 1.81 & 0.36 & 1.00 & 0.007 & 0.08 & 1.20 & 12.03 \\
\hline 0.064 & 0.05 & 1.28 & 0.82 & 2.02 & 0.32 & 1.91 & 5.73 & 7 & 0.22 & 0.81 & 1.50 & 0.50 & 1.00 & 0.004 & 0.08 & 1.68 & 16.79 \\
\hline 0.064 & 0.05 & 1.28 & 0.82 & 2.02 & 0.32 & 1.91 & 6.55 & 8 & 0.22 & 0.74 & 1.98 & 0.81 & 1.00 & 0.001 & 0.08 & 1.83 & 18.35 \\
\hline 0.064 & 0.05 & 1.28 & 0.82 & 2.02 & 0.32 & 1.91 & 7.37 & 9 & 0.23 & 0.66 & 2.59 & 1.35 & 1.00 & 0.000 & 0.08 & 2.05 & 20.50 \\
\hline 0.61 & 0.5 & 1.22 & 0.79 & 2.02 & 0.31 & 1.91 & 0.79 & 1 & 0.31 & 2.02 & 1.91 & 0.14 & 2.61 & 0.021 & 0.08 & 0.00 & 0.08 \\
\hline 0.61 & 0.5 & 1.22 & 0.79 & 2.02 & 0.31 & 1.91 & 0.95 & 1.2 & 0.26 & 1.98 & 1.92 & 0.13 & 3.85 & 0.023 & 0.08 & 0.02 & 0.15 \\
\hline 0.61 & 0.5 & 1.22 & 0.79 & 2.02 & 0.31 & 1.91 & 1.10 & 1.4 & 0.24 & 1.94 & 1.91 & 0.12 & 4.90 & 0.024 & 0.08 & 0.05 & 0.19 \\
\hline 0.61 & 0.5 & 1.22 & 0.79 & 2.02 & 0.31 & 1.91 & 1.26 & 1.6 & 0.22 & 1.89 & 1.91 & 0.11 & 5.62 & 0.025 & 0.08 & 0.09 & 0.24 \\
\hline 0.61 & 0.5 & 1.22 & 0.79 & 2.02 & 0.31 & 1.91 & 1.42 & 1.8 & 0.20 & 1.84 & 1.90 & 0.11 & 5.99 & 0.025 & 0.08 & 0.13 & 0.30 \\
\hline 0.61 & 0.5 & 1.22 & 0.79 & 2.02 & 0.31 & 1.91 & 1.58 & 2 & 0.19 & 1.79 & 1.89 & 0.11 & 6.02 & 0.025 & 0.08 & 0.17 & 0.37 \\
\hline 0.61 & 0.5 & 1.22 & 0.79 & 2.02 & 0.31 & 1.91 & 1.74 & 2.2 & 0.18 & 1.74 & 1.89 & 0.11 & 5.77 & 0.025 & 0.08 & 0.21 & 0.45 \\
\hline 0.61 & 0.5 & 1.22 & 0.79 & 2.02 & 0.31 & 1.91 & 1.89 & 2.4 & 0.17 & 1.69 & 1.91 & 0.12 & 5.30 & 0.025 & 0.08 & 0.26 & 0.56 \\
\hline 0.61 & 0.5 & 1.22 & 0.79 & 2.02 & 0.31 & 1.91 & 2.05 & 2.6 & 0.17 & 1.64 & 1.94 & 0.12 & 4.67 & 0.024 & 0.08 & 0.30 & 0.71 \\
\hline 0.61 & 0.5 & 1.22 & 0.79 & 2.02 & 0.31 & 1.91 & 2.21 & 2.8 & 0.16 & 1.60 & 2.00 & 0.13 & 3.92 & 0.023 & 0.08 & 0.33 & 0.92 \\
\hline 0.61 & 0.5 & 1.22 & 0.79 & 2.02 & 0.31 & 1.91 & 2.37 & 3 & 0.16 & 1.56 & 2.12 & 0.14 & 3.08 & 0.021 & 0.08 & 0.36 & 1.24 \\
\hline 0.61 & 0.5 & 1.22 & 0.79 & 2.02 & 0.31 & 1.91 & 2.52 & 3.2 & 0.15 & 1.54 & 2.35 & 0.15 & 2.15 & 0.019 & 0.08 & 0.37 & 1.80 \\
\hline 0.61 & 0.5 & 1.22 & 0.79 & 2.02 & 0.31 & 1.91 & 2.68 & 3.4 & 0.14 & 1.54 & 3.10 & 0.18 & 1.00 & 0.015 & 0.08 & 0.33 & 3.37 \\
\hline 0.61 & 0.5 & 1.22 & 0.79 & 2.02 & 0.31 & 1.91 & 2.84 & 3.6 & 0.14 & 1.51 & 3.17 & 0.19 & 1.00 & 0.014 & 0.08 & 0.35 & 3.59 \\
\hline 0.61 & 0.5 & 1.22 & 0.79 & 2.02 & 0.31 & 1.91 & 3.00 & 3.8 & 0.13 & 1.47 & 3.23 & 0.20 & 1.00 & 0.014 & 0.08 & 0.37 & 3.80 \\
\hline 0.61 & 0.5 & 1.22 & 0.79 & 2.02 & 0.31 & 1.91 & 3.16 & 4 & 0.14 & 1.41 & 3.40 & 0.23 & 1.00 & 0.011 & 0.08 & 0.45 & 4.57 \\
\hline 0.61 & 0.5 & 1.22 & 0.79 & 2.02 & 0.31 & 1.91 & 3.95 & 5 & 0.15 & 1.18 & 2.71 & 0.29 & 1.00 & 0.009 & 0.08 & 0.77 & 7.72 \\
\hline 0.61 & 0.5 & 1.22 & 0.79 & 2.02 & 0.31 & 1.91 & 4.73 & 6 & 0.17 & 0.98 & 2.03 & 0.37 & 1.00 & 0.007 & 0.08 & 1.20 & 12.06 \\
\hline 0.61 & 0.5 & 1.22 & 0.79 & 2.02 & 0.31 & 1.91 & 5.52 & 7 & 0.21 & 0.82 & 1.69 & 0.51 & 1.00 & 0.004 & 0.08 & 1.68 & 16.88 \\
\hline 0.61 & 0.5 & 1.22 & 0.79 & 2.02 & 0.31 & 1.91 & 6.31 & 8 & 0.21 & 0.75 & 2.30 & 0.85 & 1.00 & 0.001 & 0.08 & 1.84 & 18.42 \\
\hline 0.61 & 0.5 & 1.22 & 0.79 & 2.02 & 0.31 & 1.91 & 7.10 & 9 & 0.23 & 0.66 & 2.52 & 1.30 & 1.00 & 0.000 & 0.08 & 2.18 & 21.80 \\
\hline 0.61 & 0.5 & 1.22 & 0.79 & 2.02 & 0.31 & 1.91 & 7.89 & 10 & 0.22 & 0.62 & 4.65 & 2.68 & 1.00 & 0.000 & 0.08 & 2.21 & 22.16 \\
\hline 0.076 & 0.076 & 1.00 & 0.68 & 2.02 & 0.26 & 1.91 & 0.68 & 1 & 0.26 & 2.02 & 1.91 & 0.12 & 3.32 & 0.024 & 0.10 & 0.00 & 0.10 \\
\hline 0.076 & 0.076 & 1.00 & 0.68 & 2.02 & 0.26 & 1.91 & 0.81 & 1.2 & 0.23 & 1.98 & 1.92 & 0.11 & 4.34 & 0.025 & 0.10 & 0.03 & 0.18 \\
\hline 0.076 & 0.076 & 1.00 & 0.68 & 2.02 & 0.26 & 1.91 & 0.95 & 1.4 & 0.20 & 1.94 & 1.91 & 0.10 & 5.08 & 0.026 & 0.10 & 0.07 & 0.24 \\
\hline 0.076 & 0.076 & 1.00 & 0.68 & 2.02 & 0.26 & 1.91 & 1.09 & 1.6 & 0.19 & 1.89 & 1.91 & 0.10 & 5.53 & 0.027 & 0.10 & 0.12 & 0.32 \\
\hline 0.076 & 0.076 & 1.00 & 0.68 & 2.02 & 0.26 & 1.91 & 1.22 & 1.8 & 0.17 & 1.83 & 1.90 & 0.10 & 5.72 & 0.027 & 0.10 & 0.17 & 0.40 \\
\hline
\end{tabular}




\begin{tabular}{|c|c|c|c|c|c|c|c|c|c|c|c|c|c|c|c|c|c|}
\hline 0.076 & 0.076 & 1.00 & 0.68 & 2.02 & 0.26 & 1.91 & 1.36 & 2 & 0.16 & 1.78 & 1.90 & 0.10 & 5.67 & 0.027 & 0.10 & 0.22 & 0.49 \\
\hline 0.076 & 0.076 & 1.00 & 0.68 & 2.02 & 0.26 & 1.91 & 1.49 & 2.2 & 0.16 & 1.73 & 1.90 & 0.10 & 5.45 & 0.027 & 0.10 & 0.28 & 0.61 \\
\hline 0.076 & 0.076 & 1.00 & 0.68 & 2.02 & 0.26 & 1.91 & 1.63 & 2.4 & 0.15 & 1.68 & 1.92 & 0.10 & 5.09 & 0.026 & 0.10 & 0.33 & 0.75 \\
\hline 0.076 & 0.076 & 1.00 & 0.68 & 2.02 & 0.26 & 1.91 & 1.77 & 2.6 & 0.15 & 1.63 & 1.95 & 0.11 & 4.62 & 0.025 & 0.10 & 0.38 & 0.93 \\
\hline 0.076 & 0.076 & 1.00 & 0.68 & 2.02 & 0.26 & 1.91 & 1.90 & 2.8 & 0.14 & 1.58 & 1.99 & 0.11 & 4.09 & 0.024 & 0.10 & 0.43 & 1.16 \\
\hline 0.076 & 0.076 & 1.00 & 0.68 & 2.02 & 0.26 & 1.91 & 2.04 & 3 & 0.14 & 1.54 & 2.07 & 0.12 & 3.50 & 0.023 & 0.10 & 0.47 & 1.45 \\
\hline 0.076 & 0.076 & 1.00 & 0.68 & 2.02 & 0.26 & 1.91 & 2.17 & 3.2 & 0.14 & 1.51 & 2.19 & 0.13 & 2.90 & 0.022 & 0.10 & 0.51 & 1.85 \\
\hline 0.076 & 0.076 & 1.00 & 0.68 & 2.02 & 0.26 & 1.91 & 2.31 & 3.4 & 0.13 & 1.48 & 2.37 & 0.14 & 2.27 & 0.020 & 0.10 & 0.53 & 2.42 \\
\hline 0.076 & 0.076 & 1.00 & 0.68 & 2.02 & 0.26 & 1.91 & 2.44 & 3.6 & 0.12 & 1.46 & 2.69 & 0.16 & 1.63 & 0.018 & 0.10 & 0.52 & 3.31 \\
\hline 0.076 & 0.076 & 1.00 & 0.68 & 2.02 & 0.26 & 1.91 & 2.58 & 3.8 & 0.12 & 1.46 & 3.30 & 0.18 & 1.00 & 0.015 & 0.10 & 0.48 & 4.93 \\
\hline 0.076 & 0.076 & 1.00 & 0.68 & 2.02 & 0.26 & 1.91 & 2.72 & 4 & 0.12 & 1.42 & 3.41 & 0.20 & 1.00 & 0.013 & 0.10 & 0.53 & 5.37 \\
\hline 0.076 & 0.076 & 1.00 & 0.68 & 2.02 & 0.26 & 1.91 & 3.40 & 5 & 0.12 & 1.20 & 3.06 & 0.27 & 1.00 & 0.010 & 0.10 & 0.89 & 8.97 \\
\hline 0.076 & 0.076 & 1.00 & 0.68 & 2.02 & 0.26 & 1.91 & 4.07 & 6 & 0.15 & 0.98 & 2.23 & 0.35 & 1.00 & 0.007 & 0.10 & 1.46 & 14.68 \\
\hline 0.076 & 0.076 & 1.00 & 0.68 & 2.02 & 0.26 & 1.91 & 4.75 & 7 & 0.18 & 0.81 & 1.80 & 0.49 & 1.00 & 0.004 & 0.10 & 2.12 & 21.23 \\
\hline 0.076 & 0.076 & 1.00 & 0.68 & 2.02 & 0.26 & 1.91 & 5.43 & 8 & 0.18 & 0.74 & 2.38 & 0.78 & 1.00 & 0.001 & 0.10 & 2.30 & 22.99 \\
\hline 0.076 & 0.076 & 1.00 & 0.68 & 2.02 & 0.26 & 1.91 & 6.11 & 9 & 0.20 & 0.65 & 2.38 & 1.15 & 1.00 & 0.000 & 0.10 & 2.80 & 28.02 \\
\hline 0.051 & 0.076 & 0.67 & 0.51 & 2.02 & 0.20 & 1.91 & 0.51 & 1 & 0.20 & 2.02 & 1.91 & 0.09 & 4.19 & 0.027 & 0.16 & 0.00 & 0.17 \\
\hline 0.051 & 0.076 & 0.67 & 0.51 & 2.02 & 0.20 & 1.91 & 0.62 & 1.2 & 0.17 & 1.98 & 1.91 & 0.08 & 4.87 & 0.029 & 0.16 & 0.06 & 0.28 \\
\hline 0.051 & 0.076 & 0.67 & 0.51 & 2.02 & 0.20 & 1.91 & 0.72 & 1.4 & 0.16 & 1.93 & 1.91 & 0.08 & 5.29 & 0.029 & 0.16 & 0.13 & 0.41 \\
\hline 0.051 & 0.076 & 0.67 & 0.51 & 2.02 & 0.20 & 1.91 & 0.82 & 1.6 & 0.14 & 1.87 & 1.91 & 0.08 & 5.51 & 0.030 & 0.16 & 0.20 & 0.54 \\
\hline 0.051 & 0.076 & 0.67 & 0.51 & 2.02 & 0.20 & 1.91 & 0.93 & 1.8 & 0.13 & 1.82 & 1.90 & 0.08 & 5.56 & 0.030 & 0.16 & 0.29 & 0.69 \\
\hline 0.051 & 0.076 & 0.67 & 0.51 & 2.02 & 0.20 & 1.91 & 1.03 & 2 & 0.13 & 1.76 & 1.90 & 0.08 & 5.48 & 0.030 & 0.16 & 0.38 & 0.86 \\
\hline 0.051 & 0.076 & 0.67 & 0.51 & 2.02 & 0.20 & 1.91 & 1.13 & 2.2 & 0.12 & 1.71 & 1.91 & 0.08 & 5.30 & 0.030 & 0.16 & 0.47 & 1.06 \\
\hline 0.051 & 0.076 & 0.67 & 0.51 & 2.02 & 0.20 & 1.91 & 1.23 & 2.4 & 0.12 & 1.66 & 1.92 & 0.08 & 5.04 & 0.029 & 0.16 & 0.57 & 1.29 \\
\hline 0.051 & 0.076 & 0.67 & 0.51 & 2.02 & 0.20 & 1.91 & 1.34 & 2.6 & 0.12 & 1.60 & 1.94 & 0.09 & 4.71 & 0.028 & 0.16 & 0.66 & 1.56 \\
\hline 0.051 & 0.076 & 0.67 & 0.51 & 2.02 & 0.20 & 1.91 & 1.44 & 2.8 & 0.11 & 1.56 & 1.98 & 0.09 & 4.35 & 0.027 & 0.16 & 0.75 & 1.88 \\
\hline 0.051 & 0.076 & 0.67 & 0.51 & 2.02 & 0.20 & 1.91 & 1.54 & 3 & 0.11 & 1.51 & 2.02 & 0.10 & 3.96 & 0.027 & 0.16 & 0.83 & 2.26 \\
\hline 0.051 & 0.076 & 0.67 & 0.51 & 2.02 & 0.20 & 1.91 & 1.65 & 3.2 & 0.11 & 1.47 & 2.09 & 0.10 & 3.55 & 0.025 & 0.16 & 0.90 & 2.70 \\
\hline 0.051 & 0.076 & 0.67 & 0.51 & 2.02 & 0.20 & 1.91 & 1.75 & 3.4 & 0.11 & 1.44 & 2.18 & 0.11 & 3.13 & 0.024 & 0.16 & 0.97 & 3.24 \\
\hline 0.051 & 0.076 & 0.67 & 0.51 & 2.02 & 0.20 & 1.91 & 1.85 & 3.6 & 0.10 & 1.41 & 2.30 & 0.12 & 2.72 & 0.023 & 0.16 & 1.02 & 3.90 \\
\hline 0.051 & 0.076 & 0.67 & 0.51 & 2.02 & 0.20 & 1.91 & 1.96 & 3.8 & 0.10 & 1.38 & 2.45 & 0.13 & 2.31 & 0.021 & 0.16 & 1.05 & 4.70 \\
\hline
\end{tabular}




\begin{tabular}{|c|c|c|c|c|c|c|c|c|c|c|c|c|c|c|c|c|c|}
\hline 0.051 & 0.076 & 0.67 & 0.51 & 2.02 & 0.20 & 1.91 & 2.06 & 4 & 0.10 & 1.36 & 2.66 & 0.14 & 1.92 & 0.019 & 0.16 & 1.07 & 5.71 \\
\hline 0.051 & 0.076 & 0.67 & 0.51 & 2.02 & 0.20 & 1.91 & 2.57 & 5 & 0.09 & 1.23 & 3.70 & 0.22 & 1.00 & 0.012 & 0.16 & 1.22 & 12.38 \\
\hline 0.051 & 0.076 & 0.67 & 0.51 & 2.02 & 0.20 & 1.91 & 3.09 & 6 & 0.10 & 1.02 & 2.99 & 0.30 & 1.00 & 0.008 & 0.16 & 1.99 & 19.99 \\
\hline 0.051 & 0.076 & 0.67 & 0.51 & 2.02 & 0.20 & 1.91 & 3.60 & 7 & 0.12 & 0.86 & 2.74 & 0.44 & 1.00 & 0.004 & 0.16 & 2.75 & 27.61 \\
\hline 0.064 & 0.1 & 0.64 & 0.50 & 2.02 & 0.19 & 1.91 & 0.50 & 1 & 0.19 & 2.02 & 1.91 & 0.09 & 4.26 & 0.028 & 0.17 & 0.00 & 0.18 \\
\hline 0.064 & 0.1 & 0.64 & 0.50 & 2.02 & 0.19 & 1.91 & 0.60 & 1.2 & 0.17 & 1.98 & 1.91 & 0.08 & 4.91 & 0.029 & 0.17 & 0.06 & 0.30 \\
\hline 0.064 & 0.1 & 0.64 & 0.50 & 2.02 & 0.19 & 1.91 & 0.70 & 1.4 & 0.15 & 1.93 & 1.91 & 0.08 & 5.31 & 0.030 & 0.17 & 0.13 & 0.43 \\
\hline 0.064 & 0.1 & 0.64 & 0.50 & 2.02 & 0.19 & 1.91 & 0.80 & 1.6 & 0.14 & 1.87 & 1.91 & 0.08 & 5.51 & 0.030 & 0.17 & 0.22 & 0.57 \\
\hline 0.064 & 0.1 & 0.64 & 0.50 & 2.02 & 0.19 & 1.91 & 0.90 & 1.8 & 0.13 & 1.82 & 1.90 & 0.07 & 5.56 & 0.030 & 0.17 & 0.31 & 0.73 \\
\hline 0.064 & 0.1 & 0.64 & 0.50 & 2.02 & 0.19 & 1.91 & 1.00 & 2 & 0.12 & 1.76 & 1.90 & 0.08 & 5.47 & 0.030 & 0.17 & 0.41 & 0.92 \\
\hline 0.064 & 0.1 & 0.64 & 0.50 & 2.02 & 0.19 & 1.91 & 1.10 & 2.2 & 0.12 & 1.71 & 1.91 & 0.08 & 5.29 & 0.030 & 0.17 & 0.51 & 1.13 \\
\hline 0.064 & 0.1 & 0.64 & 0.50 & 2.02 & 0.19 & 1.91 & 1.20 & 2.4 & 0.12 & 1.65 & 1.92 & 0.08 & 5.04 & 0.029 & 0.17 & 0.61 & 1.38 \\
\hline 0.064 & 0.1 & 0.64 & 0.50 & 2.02 & 0.19 & 1.91 & 1.30 & 2.6 & 0.11 & 1.60 & 1.94 & 0.08 & 4.73 & 0.029 & 0.17 & 0.70 & 1.66 \\
\hline 0.064 & 0.1 & 0.64 & 0.50 & 2.02 & 0.19 & 1.91 & 1.40 & 2.8 & 0.11 & 1.55 & 1.97 & 0.09 & 4.38 & 0.028 & 0.17 & 0.80 & 2.00 \\
\hline 0.064 & 0.1 & 0.64 & 0.50 & 2.02 & 0.19 & 1.91 & 1.50 & 3 & 0.11 & 1.51 & 2.02 & 0.10 & 4.00 & 0.027 & 0.17 & 0.89 & 2.39 \\
\hline 0.064 & 0.1 & 0.64 & 0.50 & 2.02 & 0.19 & 1.91 & 1.60 & 3.2 & 0.11 & 1.47 & 2.08 & 0.10 & 3.60 & 0.026 & 0.17 & 0.97 & 2.86 \\
\hline 0.064 & 0.1 & 0.64 & 0.50 & 2.02 & 0.19 & 1.91 & 1.70 & 3.4 & 0.10 & 1.43 & 2.17 & 0.11 & 3.20 & 0.024 & 0.17 & 1.04 & 3.41 \\
\hline 0.064 & 0.1 & 0.64 & 0.50 & 2.02 & 0.19 & 1.91 & 1.80 & 3.6 & 0.10 & 1.40 & 2.28 & 0.12 & 2.80 & 0.023 & 0.17 & 1.09 & 4.08 \\
\hline 0.064 & 0.1 & 0.64 & 0.50 & 2.02 & 0.19 & 1.91 & 1.90 & 3.8 & 0.10 & 1.37 & 2.42 & 0.13 & 2.40 & 0.022 & 0.17 & 1.14 & 4.89 \\
\hline 0.064 & 0.1 & 0.64 & 0.50 & 2.02 & 0.19 & 1.91 & 2.00 & 4 & 0.10 & 1.35 & 2.62 & 0.14 & 2.02 & 0.020 & 0.17 & 1.16 & 5.89 \\
\hline 0.064 & 0.1 & 0.64 & 0.50 & 2.02 & 0.19 & 1.91 & 2.10 & 4.2 & 0.09 & 1.33 & 2.87 & 0.15 & 1.66 & 0.018 & 0.17 & 1.16 & 7.16 \\
\hline 0.064 & 0.1 & 0.64 & 0.50 & 2.02 & 0.19 & 1.91 & 2.20 & 4.4 & 0.09 & 1.32 & 3.22 & 0.16 & 1.31 & 0.016 & 0.17 & 1.13 & 8.81 \\
\hline 0.064 & 0.1 & 0.64 & 0.50 & 2.02 & 0.19 & 1.91 & 2.50 & 5 & 0.09 & 1.23 & 3.75 & 0.21 & 1.00 & 0.012 & 0.17 & 1.28 & 12.96 \\
\hline 0.064 & 0.1 & 0.64 & 0.50 & 2.02 & 0.19 & 1.91 & 2.99 & 6 & 0.10 & 1.03 & 3.19 & 0.30 & 1.00 & 0.008 & 0.17 & 2.02 & 20.36 \\
\hline 0.064 & 0.1 & 0.64 & 0.50 & 2.02 & 0.19 & 1.91 & 3.49 & 7 & 0.11 & 0.87 & 2.95 & 0.44 & 1.00 & 0.004 & 0.17 & 2.80 & 28.10 \\
\hline 0.064 & 0.1 & 0.64 & 0.50 & 2.02 & 0.19 & 1.91 & 3.99 & 8 & 0.13 & 0.74 & 2.66 & 0.64 & 1.00 & 0.002 & 0.17 & 3.74 & 37.48 \\
\hline 0.064 & 0.1 & 0.64 & 0.50 & 2.02 & 0.19 & 1.91 & 4.49 & 9 & 0.16 & 0.63 & 2.34 & 0.92 & 1.00 & 0.000 & 0.17 & 4.90 & 49.08 \\
\hline 0.064 & 0.1 & 0.64 & 0.50 & 2.02 & 0.19 & 1.91 & 4.99 & 10 & 0.17 & 0.57 & 2.60 & 1.36 & 1.00 & 0.000 & 0.17 & 5.57 & 55.72 \\
\hline 0.107 & 0.206 & 0.52 & 0.44 & 2.02 & 0.17 & 1.91 & 0.44 & 1 & 0.17 & 2.02 & 1.91 & 0.08 & 4.52 & 0.029 & 0.23 & 0.00 & 0.24 \\
\hline 0.107 & 0.206 & 0.52 & 0.44 & 2.02 & 0.17 & 1.91 & 0.53 & 1.2 & 0.15 & 1.98 & 1.91 & 0.07 & 5.06 & 0.031 & 0.23 & 0.08 & 0.40 \\
\hline 0.107 & 0.206 & 0.52 & 0.44 & 2.02 & 0.17 & 1.91 & 0.61 & 1.4 & 0.13 & 1.92 & 1.91 & 0.07 & 5.38 & 0.031 & 0.23 & 0.18 & 0.58 \\
\hline
\end{tabular}




\begin{tabular}{|c|c|c|c|c|c|c|c|c|c|c|c|c|c|c|c|c|c|}
\hline 0.107 & 0.206 & 0.52 & 0.44 & 2.02 & 0.17 & 1.91 & 0.70 & 1.6 & 0.12 & 1.87 & 1.91 & 0.07 & 5.53 & 0.032 & 0.23 & 0.30 & 0.77 \\
\hline 0.107 & 0.206 & 0.52 & 0.44 & 2.02 & 0.17 & 1.91 & 0.79 & 1.8 & 0.12 & 1.81 & 1.90 & 0.07 & 5.55 & 0.032 & 0.23 & 0.42 & 0.99 \\
\hline 0.107 & 0.206 & 0.52 & 0.44 & 2.02 & 0.17 & 1.91 & 0.88 & 2 & 0.11 & 1.76 & 1.90 & 0.07 & 5.46 & 0.031 & 0.23 & 0.55 & 1.24 \\
\hline 0.107 & 0.206 & 0.52 & 0.44 & 2.02 & 0.17 & 1.91 & 0.97 & 2.2 & 0.11 & 1.70 & 1.91 & 0.07 & 5.30 & 0.031 & 0.23 & 0.68 & 1.52 \\
\hline 0.107 & 0.206 & 0.52 & 0.44 & 2.02 & 0.17 & 1.91 & 1.05 & 2.4 & 0.10 & 1.64 & 1.92 & 0.07 & 5.07 & 0.031 & 0.23 & 0.82 & 1.85 \\
\hline 0.107 & 0.206 & 0.52 & 0.44 & 2.02 & 0.17 & 1.91 & 1.14 & 2.6 & 0.10 & 1.59 & 1.94 & 0.08 & 4.80 & 0.030 & 0.23 & 0.95 & 2.22 \\
\hline 0.107 & 0.206 & 0.52 & 0.44 & 2.02 & 0.17 & 1.91 & 1.23 & 2.8 & 0.10 & 1.54 & 1.97 & 0.08 & 4.49 & 0.029 & 0.23 & 1.09 & 2.65 \\
\hline 0.107 & 0.206 & 0.52 & 0.44 & 2.02 & 0.17 & 1.91 & 1.32 & 3 & 0.10 & 1.50 & 2.01 & 0.09 & 4.16 & 0.028 & 0.23 & 1.21 & 3.13 \\
\hline 0.107 & 0.206 & 0.52 & 0.44 & 2.02 & 0.17 & 1.91 & 1.40 & 3.2 & 0.09 & 1.46 & 2.06 & 0.09 & 3.81 & 0.027 & 0.23 & 1.32 & 3.70 \\
\hline 0.107 & 0.206 & 0.52 & 0.44 & 2.02 & 0.17 & 1.91 & 1.49 & 3.4 & 0.09 & 1.42 & 2.13 & 0.10 & 3.45 & 0.026 & 0.23 & 1.43 & 4.35 \\
\hline 0.107 & 0.206 & 0.52 & 0.44 & 2.02 & 0.17 & 1.91 & 1.58 & 3.6 & 0.09 & 1.38 & 2.22 & 0.11 & 3.09 & 0.025 & 0.23 & 1.51 & 5.11 \\
\hline 0.107 & 0.206 & 0.52 & 0.44 & 2.02 & 0.17 & 1.91 & 1.67 & 3.8 & 0.09 & 1.35 & 2.33 & 0.11 & 2.74 & 0.023 & 0.23 & 1.59 & 6.01 \\
\hline 0.107 & 0.206 & 0.52 & 0.44 & 2.02 & 0.17 & 1.91 & 1.75 & 4 & 0.09 & 1.33 & 2.47 & 0.12 & 2.40 & 0.022 & 0.23 & 1.64 & 7.06 \\
\hline 0.107 & 0.206 & 0.52 & 0.44 & 2.02 & 0.17 & 1.91 & 2.19 & 5 & 0.07 & 1.24 & 3.86 & 0.19 & 1.00 & 0.013 & 0.23 & 1.61 & 16.29 \\
\hline 0.107 & 0.206 & 0.52 & 0.44 & 2.02 & 0.17 & 1.91 & 2.63 & 6 & 0.08 & 1.04 & 3.49 & 0.27 & 1.00 & 0.009 & 0.23 & 2.48 & 24.98 \\
\hline 0.107 & 0.206 & 0.52 & 0.44 & 2.02 & 0.17 & 1.91 & 3.07 & 7 & 0.09 & 0.90 & 3.52 & 0.40 & 1.00 & 0.005 & 0.23 & 3.30 & 33.10 \\
\hline 0.107 & 0.206 & 0.52 & 0.44 & 2.02 & 0.17 & 1.91 & 3.51 & 8 & 0.11 & 0.75 & 2.85 & 0.57 & 1.00 & 0.002 & 0.23 & 4.72 & 47.31 \\
\hline 0.107 & 0.206 & 0.52 & 0.44 & 2.02 & 0.17 & 1.91 & 3.95 & 9 & 0.14 & 0.63 & 2.40 & 0.83 & 1.00 & 0.001 & 0.23 & 6.39 & 63.97 \\
\hline 0.107 & 0.206 & 0.52 & 0.44 & 2.02 & 0.17 & 1.91 & 4.39 & 10 & 0.16 & 0.56 & 2.48 & 1.21 & 1.00 & 0.000 & 0.23 & 7.53 & 75.27 \\
\hline 0.051 & 0.127 & 0.40 & 0.38 & 2.02 & 0.15 & 1.91 & 0.38 & 1 & 0.15 & 2.02 & 1.91 & 0.07 & 4.75 & 0.031 & 0.33 & 0.00 & 0.34 \\
\hline 0.051 & 0.127 & 0.40 & 0.38 & 2.02 & 0.15 & 1.91 & 0.46 & 1.2 & 0.13 & 1.97 & 1.91 & 0.06 & 5.19 & 0.032 & 0.33 & 0.12 & 0.58 \\
\hline 0.051 & 0.127 & 0.40 & 0.38 & 2.02 & 0.15 & 1.91 & 0.53 & 1.4 & 0.12 & 1.92 & 1.91 & 0.06 & 5.44 & 0.033 & 0.33 & 0.27 & 0.84 \\
\hline 0.051 & 0.127 & 0.40 & 0.38 & 2.02 & 0.15 & 1.91 & 0.61 & 1.6 & 0.11 & 1.87 & 1.90 & 0.06 & 5.55 & 0.033 & 0.33 & 0.44 & 1.13 \\
\hline 0.051 & 0.127 & 0.40 & 0.38 & 2.02 & 0.15 & 1.91 & 0.68 & 1.8 & 0.10 & 1.81 & 1.90 & 0.06 & 5.55 & 0.033 & 0.33 & 0.62 & 1.46 \\
\hline 0.051 & 0.127 & 0.40 & 0.38 & 2.02 & 0.15 & 1.91 & 0.76 & 2 & 0.10 & 1.75 & 1.90 & 0.06 & 5.47 & 0.033 & 0.33 & 0.82 & 1.83 \\
\hline 0.051 & 0.127 & 0.40 & 0.38 & 2.02 & 0.15 & 1.91 & 0.84 & 2.2 & 0.09 & 1.69 & 1.91 & 0.06 & 5.32 & 0.032 & 0.33 & 1.02 & 2.24 \\
\hline 0.051 & 0.127 & 0.40 & 0.38 & 2.02 & 0.15 & 1.91 & 0.91 & 2.4 & 0.09 & 1.64 & 1.92 & 0.06 & 5.12 & 0.032 & 0.33 & 1.22 & 2.71 \\
\hline 0.051 & 0.127 & 0.40 & 0.38 & 2.02 & 0.15 & 1.91 & 0.99 & 2.6 & 0.09 & 1.58 & 1.93 & 0.07 & 4.88 & 0.031 & 0.33 & 1.42 & 3.24 \\
\hline 0.051 & 0.127 & 0.40 & 0.38 & 2.02 & 0.15 & 1.91 & 1.06 & 2.8 & 0.09 & 1.53 & 1.96 & 0.07 & 4.61 & 0.030 & 0.33 & 1.61 & 3.83 \\
\hline 0.051 & 0.127 & 0.40 & 0.38 & 2.02 & 0.15 & 1.91 & 1.14 & 3 & 0.08 & 1.49 & 1.99 & 0.08 & 4.32 & 0.030 & 0.33 & 1.80 & 4.50 \\
\hline 0.051 & 0.127 & 0.40 & 0.38 & 2.02 & 0.15 & 1.91 & 1.22 & 3.2 & 0.08 & 1.44 & 2.04 & 0.08 & 4.01 & 0.029 & 0.33 & 1.98 & 5.26 \\
\hline
\end{tabular}




\begin{tabular}{|c|c|c|c|c|c|c|c|c|c|c|c|c|c|c|c|c|c|}
\hline 0.051 & 0.127 & 0.40 & 0.38 & 2.02 & 0.15 & 1.91 & 1.29 & 3.4 & 0.08 & 1.40 & 2.09 & 0.09 & 3.70 & 0.028 & 0.33 & 2.14 & 6.12 \\
\hline 0.051 & 0.127 & 0.40 & 0.38 & 2.02 & 0.15 & 1.91 & 1.37 & 3.6 & 0.08 & 1.37 & 2.17 & 0.09 & 3.38 & 0.026 & 0.33 & 2.29 & 7.10 \\
\hline 0.051 & 0.127 & 0.40 & 0.38 & 2.02 & 0.15 & 1.91 & 1.44 & 3.8 & 0.08 & 1.33 & 2.26 & 0.10 & 3.06 & 0.025 & 0.33 & 2.41 & 8.22 \\
\hline 0.051 & 0.127 & 0.40 & 0.38 & 2.02 & 0.15 & 1.91 & 1.52 & 4 & 0.08 & 1.30 & 2.36 & 0.11 & 2.74 & 0.024 & 0.33 & 2.52 & 9.50 \\
\hline 0.051 & 0.127 & 0.40 & 0.38 & 2.02 & 0.15 & 1.91 & 1.90 & 5 & 0.07 & 1.21 & 3.41 & 0.16 & 1.34 & 0.016 & 0.33 & 2.61 & 19.79 \\
\hline 0.051 & 0.127 & 0.40 & 0.38 & 2.02 & 0.15 & 1.91 & 2.28 & 6 & 0.07 & 1.06 & 3.85 & 0.24 & 1.00 & 0.011 & 0.33 & 3.29 & 33.11 \\
\hline 0.051 & 0.127 & 0.40 & 0.38 & 2.02 & 0.15 & 1.91 & 2.66 & 7 & 0.08 & 0.91 & 3.75 & 0.35 & 1.00 & 0.006 & 0.33 & 4.49 & 45.09 \\
\hline 0.051 & 0.127 & 0.40 & 0.38 & 2.02 & 0.15 & 1.91 & 3.04 & 8 & 0.09 & 0.76 & 3.12 & 0.50 & 1.00 & 0.003 & 0.33 & 6.39 & 64.10 \\
\hline 0.051 & 0.127 & 0.40 & 0.38 & 2.02 & 0.15 & 1.91 & 3.42 & 9 & 0.12 & 0.64 & 2.53 & 0.73 & 1.00 & 0.001 & 0.33 & 8.92 & 89.27 \\
\hline 0.051 & 0.127 & 0.40 & 0.38 & 2.02 & 0.15 & 1.91 & 3.80 & 10 & 0.14 & 0.56 & 2.41 & 1.07 & 1.00 & 0.000 & 0.33 & 11.01 & 110.1 \\
\hline 0.035 & 0.127 & 0.28 & 0.32 & 2.02 & 0.12 & 1.91 & 0.32 & 1 & 0.12 & 2.02 & 1.91 & 0.06 & 4.98 & 0.033 & 0.59 & 0.00 & 0.62 \\
\hline 0.035 & 0.127 & 0.28 & 0.32 & 2.02 & 0.12 & 1.91 & 0.38 & 1.2 & 0.11 & 1.97 & 1.91 & 0.05 & 5.32 & 0.034 & 0.59 & 0.23 & 1.06 \\
\hline 0.035 & 0.127 & 0.28 & 0.32 & 2.02 & 0.12 & 1.91 & 0.44 & 1.4 & 0.10 & 1.92 & 1.91 & 0.05 & 5.51 & 0.034 & 0.59 & 0.51 & 1.54 \\
\hline 0.035 & 0.127 & 0.28 & 0.32 & 2.02 & 0.12 & 1.91 & 0.51 & 1.6 & 0.09 & 1.86 & 1.90 & 0.05 & 5.58 & 0.034 & 0.59 & 0.82 & 2.08 \\
\hline 0.035 & 0.127 & 0.28 & 0.32 & 2.02 & 0.12 & 1.91 & 0.57 & 1.8 & 0.08 & 1.80 & 1.90 & 0.05 & 5.57 & 0.034 & 0.59 & 1.16 & 2.69 \\
\hline 0.035 & 0.127 & 0.28 & 0.32 & 2.02 & 0.12 & 1.91 & 0.63 & 2 & 0.08 & 1.74 & 1.90 & 0.05 & 5.49 & 0.034 & 0.59 & 1.52 & 3.37 \\
\hline 0.035 & 0.127 & 0.28 & 0.32 & 2.02 & 0.12 & 1.91 & 0.70 & 2.2 & 0.08 & 1.68 & 1.90 & 0.05 & 5.36 & 0.034 & 0.59 & 1.89 & 4.13 \\
\hline 0.035 & 0.127 & 0.28 & 0.32 & 2.02 & 0.12 & 1.91 & 0.76 & 2.4 & 0.08 & 1.63 & 1.91 & 0.05 & 5.19 & 0.033 & 0.59 & 2.27 & 4.97 \\
\hline 0.035 & 0.127 & 0.28 & 0.32 & 2.02 & 0.12 & 1.91 & 0.82 & 2.6 & 0.07 & 1.57 & 1.93 & 0.06 & 4.98 & 0.033 & 0.59 & 2.65 & 5.91 \\
\hline 0.035 & 0.127 & 0.28 & 0.32 & 2.02 & 0.12 & 1.91 & 0.89 & 2.8 & 0.07 & 1.52 & 1.95 & 0.06 & 4.75 & 0.032 & 0.59 & 3.02 & 6.95 \\
\hline 0.035 & 0.127 & 0.28 & 0.32 & 2.02 & 0.12 & 1.91 & 0.95 & 3 & 0.07 & 1.47 & 1.98 & 0.07 & 4.50 & 0.031 & 0.59 & 3.38 & 8.10 \\
\hline 0.035 & 0.127 & 0.28 & 0.32 & 2.02 & 0.12 & 1.91 & 1.01 & 3.2 & 0.07 & 1.43 & 2.01 & 0.07 & 4.24 & 0.030 & 0.59 & 3.73 & 9.39 \\
\hline 0.035 & 0.127 & 0.28 & 0.32 & 2.02 & 0.12 & 1.91 & 1.08 & 3.4 & 0.07 & 1.39 & 2.06 & 0.08 & 3.96 & 0.029 & 0.59 & 4.05 & 10.81 \\
\hline 0.035 & 0.127 & 0.28 & 0.32 & 2.02 & 0.12 & 1.91 & 1.14 & 3.6 & 0.07 & 1.35 & 2.12 & 0.08 & 3.68 & 0.028 & 0.59 & 4.35 & 12.40 \\
\hline 0.035 & 0.127 & 0.28 & 0.32 & 2.02 & 0.12 & 1.91 & 1.20 & 3.8 & 0.07 & 1.31 & 2.19 & 0.09 & 3.39 & 0.027 & 0.59 & 4.62 & 14.18 \\
\hline 0.035 & 0.127 & 0.28 & 0.32 & 2.02 & 0.12 & 1.91 & 1.27 & 4 & 0.07 & 1.28 & 2.27 & 0.09 & 3.11 & 0.026 & 0.59 & 4.85 & 16.16 \\
\hline 0.035 & 0.127 & 0.28 & 0.32 & 2.02 & 0.12 & 1.91 & 1.58 & 5 & 0.06 & 1.17 & 3.00 & 0.14 & 1.80 & 0.019 & 0.59 & 5.40 & 30.48 \\
\hline 0.035 & 0.127 & 0.28 & 0.32 & 2.02 & 0.12 & 2.91 & 1.90 & 6 & 0.06 & 1.08 & 4.18 & 0.20 & 1.00 & 0.012 & 0.59 & 5.42 & 54.68 \\
\hline 0.035 & 0.127 & 0.28 & 0.32 & 2.02 & 0.12 & 3.91 & 2.22 & 7 & 0.06 & 0.93 & 4.07 & 0.29 & 1.00 & 0.008 & 0.59 & 7.47 & 75.14 \\
\hline 0.035 & 0.127 & 0.28 & 0.32 & 2.02 & 0.12 & 4.91 & 2.53 & 8 & 0.07 & 0.78 & 3.51 & 0.42 & 1.00 & 0.004 & 0.59 & 10.52 & 105.5 \\
\hline 0.035 & 0.127 & 0.28 & 0.32 & 2.02 & 0.12 & 5.91 & 2.85 & 9 & 0.09 & 0.65 & 2.81 & 0.61 & 1.00 & 0.002 & 0.59 & 14.93 & 149.5 \\
\hline
\end{tabular}




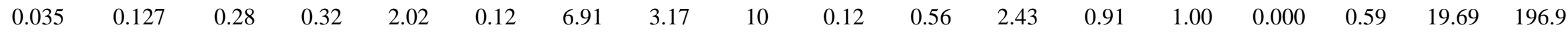

Note:

$$
\begin{aligned}
r_{i}^{\prime} & =r_{i} / H ; \\
h_{i}^{\prime} & =h_{i} / H \\
v_{i}^{\prime} & =v_{i} / \sqrt{g H} ; \\
\theta_{i} & =\left(T_{i}-T_{\infty}\right) / T_{\infty} ; \\
\mathrm{Ri}_{i} & =g \theta_{i} h_{i}^{\prime} / v_{i}{ }^{2} ; \\
m_{i}^{*} & =\dot{m}_{i} /\left(\rho_{\infty} g^{1 / 2} D^{5 / 2}\right) ; \\
Q_{D 3}^{*} & =\left(\frac{m_{p}^{*}}{n_{p}}+\frac{m_{j}^{*}}{n_{j}}\right) \lambda .
\end{aligned}
$$


Table 4. Direct comparison of theoretical prediction vs. data for flame length, $r_{f}$.

Experimental Data Theoretical Predictions Relative Error (\%)

\begin{tabular}{|c|c|c|c|c|c|c|}
\hline$D / H$ & $Q_{D_{1}}^{*}$ & $r_{f}^{\prime}$ & $Q_{D_{4}}^{*}$ & $r_{f}^{\prime}$ & Above & Below \\
\hline 3.2 & 3.48 & 7.04 & 3.30 & 7.62 & 8.20 & \\
\hline 1.4 & 0.54 & 1.58 & 0.52 & 2.21 & 39.65 & \\
\hline 1.4 & 0.66 & 2.04 & 0.64 & 2.31 & 13.41 & \\
\hline 1.4 & 0.78 & 2.50 & 0.76 & 2.43 & & -2.92 \\
\hline 1.4 & 1.88 & 3.95 & 1.83 & 2.71 & & -31.30 \\
\hline 1.28 & 3.51 & 2.32 & 3.36 & 2.94 & 26.39 & \\
\hline 1.28 & 5.53 & 3.18 & 5.29 & 3.50 & 10.12 & \\
\hline 1.28 & 6.05 & 3.64 & 5.81 & 3.68 & 1.02 & \\
\hline 1.28 & 6.60 & 4.04 & 6.09 & 3.74 & & -7.52 \\
\hline 1.28 & 6.32 & 3.90 & 6.38 & 3.80 & & -2.64 \\
\hline 1.28 & 7.17 & 4.50 & 6.89 & 3.92 & & -12.87 \\
\hline 1.28 & 7.73 & 5.06 & 7.57 & 4.05 & & -20.00 \\
\hline 1.28 & 8.35 & 5.74 & 8.06 & 4.18 & & -27.16 \\
\hline 1.22 & 0.32 & 1.40 & 0.31 & 1.47 & 5.29 & \\
\hline 1.22 & 0.39 & 1.60 & 0.38 & 1.60 & & -0.19 \\
\hline 1.22 & 0.45 & 1.80 & 0.44 & 1.76 & & -2.28 \\
\hline 1.22 & 0.50 & 1.80 & 0.49 & 1.82 & 0.89 & \\
\hline 1.22 & 0.55 & 2.00 & 0.54 & 1.91 & & -4.70 \\
\hline 1.22 & 0.63 & 2.20 & 0.61 & 1.97 & & -10.55 \\
\hline 1.22 & 0.71 & 2.20 & 0.69 & 2.07 & & -6.14 \\
\hline 1.22 & 0.78 & 2.20 & 0.76 & 2.10 & & -4.64 \\
\hline 1.22 & 0.87 & 2.20 & 0.85 & 2.17 & & -1.50 \\
\hline 1 & 0.67 & 1.25 & 0.66 & 1.52 & 21.52 & \\
\hline 1 & 0.86 & 1.33 & 0.85 & 1.67 & 25.89 & \\
\hline 1 & 1.94 & 2.50 & 1.89 & 2.16 & & -13.48 \\
\hline 0.67 & 0.94 & 0.67 & 0.95 & 1.06 & 58.56 & \\
\hline 0.67 & 1.08 & 0.79 & 1.08 & 1.14 & 43.77 & \\
\hline 0.67 & 1.20 & 0.92 & 1.22 & 1.19 & 29.31 & \\
\hline 0.67 & 2.46 & 1.21 & 2.50 & 1.59 & 31.35 & \\
\hline 0.64 & 3.48 & 1.50 & 3.37 & 1.64 & 9.77 & \\
\hline 0.64 & 3.47 & 1.34 & 3.37 & 1.64 & 22.46 & \\
\hline 0.64 & 6.56 & 2.15 & 6.39 & 2.02 & & -5.89 \\
\hline 0.64 & 9.52 & 2.67 & 9.30 & 2.23 & & -16.64 \\
\hline 0.6 & 0.67 & 0.52 & 0.63 & 0.76 & 45.95 & \\
\hline 0.6 & 0.86 & 0.65 & 0.84 & 0.85 & 31.46 & \\
\hline
\end{tabular}




$\begin{array}{ccccccc}0.6 & 0.92 & 0.80 & 0.91 & 0.89 & 12.01 & \\ 0.6 & 1.94 & 1.47 & 1.89 & 1.27 & & -13.82 \\ 0.52 & 0.66 & 0.71 & 0.64 & 0.65 & & -8.95 \\ 0.52 & 0.78 & 0.74 & 0.77 & 0.70 & & -5.21 \\ 0.52 & 1.88 & 1.42 & 1.86 & 1.05 & & -26.28 \\ 0.46 & 1.43 & 0.46 & 1.37 & 0.77 & 67.09 & \\ 0.46 & 1.60 & 0.61 & 1.41 & 0.79 & 31.30 & \\ 0.46 & 1.40 & 0.75 & 1.57 & 0.83 & 11.20 & \\ 0.46 & 3.03 & 1.07 & 2.93 & 1.10 & 2.83 & \\ 0.4 & 1.20 & 0.47 & 1.19 & 0.60 & 27.87 & \\ 0.29 & 1.94 & 0.48 & 1.90 & 0.50 & 4.23 & \\ 0.28 & 1.40 & 0.32 & 1.35 & 0.41 & 26.94 & -2.18 \\ 0.28 & 3.03 & 0.61 & 2.98 & 0.59 & & -10.31\end{array}$




\section{Appendix E: Simple Correlation}

Figure 4 shows the full results for $r_{f}^{\prime}$ vs. $Q_{\mathrm{D}}^{*}$, and Figure 5 shows a very good agreement with experiments. However, as one may have noticed, to obtain the full results requires a complex process and solving a set of complicated equations that are related to each other. The relation for $r_{f}^{\prime}$ vs. $Q_{D}^{*}$ is neither direct nor intuitive. Observing Figure 4, we discovered that the slopes of the theory lines for different $D / H$ values do not have big variations. So we seek to further investigate Figure 4, and hope to carry out a simpler equation, in the form of

$r_{f} / H=a Q_{D}^{* b}$

where $b$ is the average slope of the theory lines in Figure 12 under $\log$ scale, and $a$ should contain $D / H$ factor.

The trend lines in Figure 12 show an average value of the slopes to be 0.369 . Thus,

$b \approx 0.369 \approx 2 / 5$

Substitute $Q_{D}^{*}=1$ to Eq. (E-1) and examine relations between $r_{f} / H$ and $D / H$ from the theory lines in Figure 12.We found the correlation of theory lines to be

$r_{f} / H=1.62(D / H)^{1.087}$, for $Q_{D}^{*}=1$.

We can see that the $H$ factor can almost be neglected,

$r_{f}=1.62 D$, for $Q_{D}^{*}=1$.

Therefore, the simple correlation for $r_{f}^{\prime}$ vs. $Q_{D}^{*}$ becomes

$r_{f} / D=1.62 Q_{D}^{* 2 / 5}, H_{f} \geq H$. 


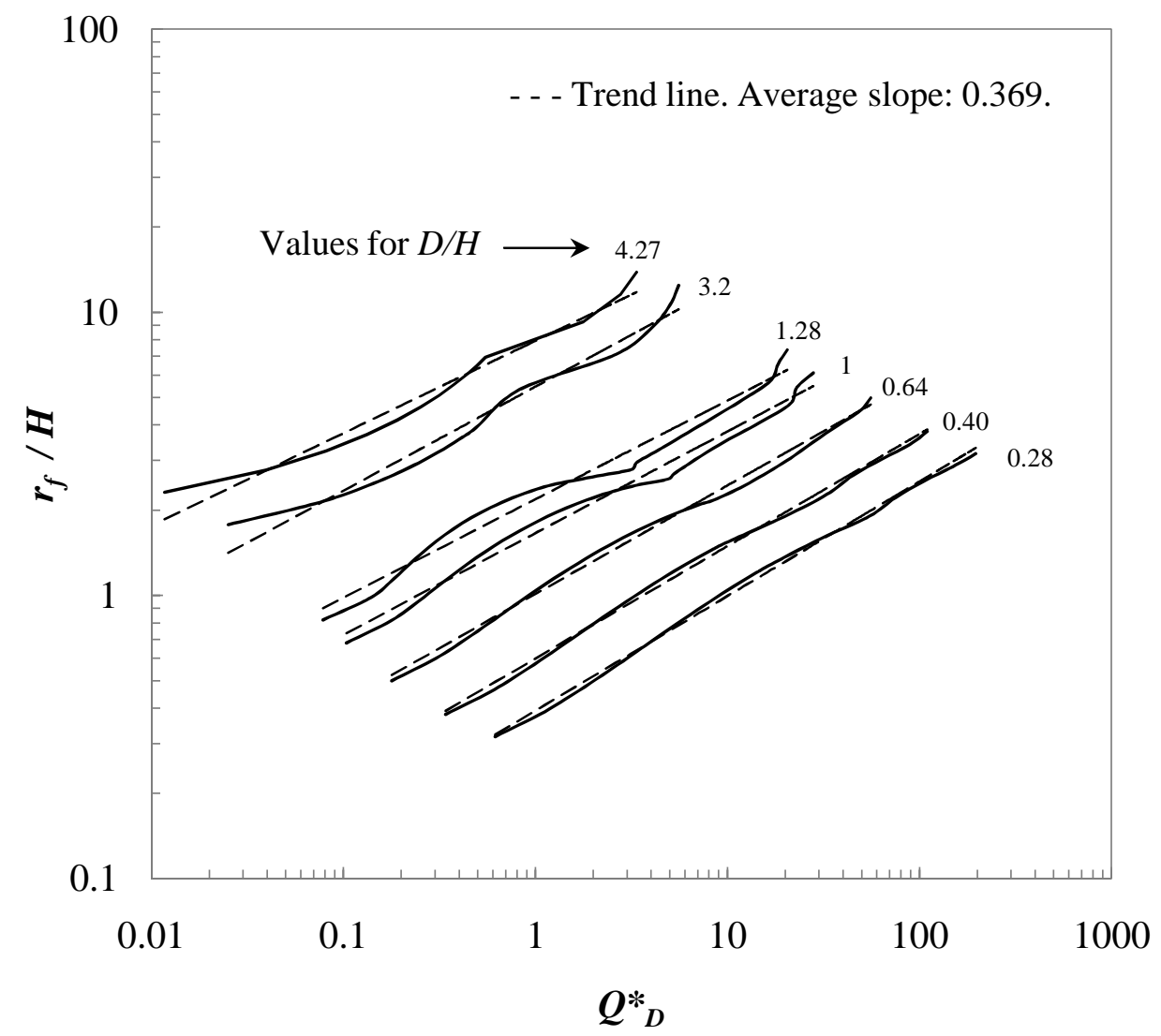

Figure 12. Correlations of theoretical results for radial flame length.

Figure 6 shows the data agreement with the simple equation correlated from the full theory. The accuracy is almost as good as the full results shown in Figure 5. $H$ effect disappeared in this simple correlation. 


\section{Bibliography}

1. Grove, B. J. and Quintiere, J. G., A Unified Analysis for Fire Plumes, Proceedings of the 27th Symposium (International) on Combustion, The Combustion Institute, 1998, pp. 27572766.

2. You, H. Z. and Faeth, G. M., Ceiling Heat Transfer during Fire Plume and Fire Impingement, Fire and Materials, Vol. 3, no.3, 1979, 140-147.

3. Babrauskas, V., Flame Lengths Under Ceilings, Fire and Materials, Vol. 4, no.3, 1980, 119126.

4. Gross, D., Measurement of Flame Lengths under Ceilings, Fire Safety Journal, 15 (1989), $31-44$.

5. Kokkala, M. A. and Rinkinen, W. J., "Some Observations on the Shape of Impinging Diffusion Flames”, NBSIR 87-3505, US DOC, Nat. Bur. Stand., January 1987.

6. Alpert, R. L., "Fire Induced Turbulent Ceiling Jet”, Tech. Rep. No. 19722-2, Factory Mutual Corp., Norwood, MA (1971).

7. You, H-Z, An Investigation of Fire Plume Impingement on a Horizontal Ceiling, 1 - Plume Region, Fire and Materials, Vol. 8, no.1, 1984, 28-39.

8. You, H-Z, An Investigation of Fire Plume Impingement on a Horizontal Ceiling, 2 Impingement and Ceiling-jet Regions, Fire and Materials, Vol. 9, no.1, 1985, 46-57.

9. Heskestad, G. and Hamada, T., Ceiling Jets of Strong Fire Plumes, Fire Safety Journal, Vol. $21,1993,69-82$ 
10. You, H-Z, "An Investigation of Fire Plume Impingement on a Horizontal Ceiling", $\mathrm{PhD}$ Thesis, The Pennsylvania State University, 1981.

11. Yao, X. and Marshall, A.W., Characterizing Turbulent Ceiling Jet Dynamics with Salt-water Modeling, Proceedings of the $8^{\text {th }}$ International Symposium on Fire Safety Science, 2005, pp. 927-938.

12. Turner, J. S., Buoyancy Effects in Fluids, Cambridge University Press, 1973.

13. SFPE Handbook of Fire Protection Engineering, Third Edition, National Fire Protection Association, 1995.

14. Heskestad, G., Turbulent Jet Diffusion Flames: Consolidation of Flame Height Data", Combustion and Flame, Vol. 118, no. 1, 1999, 51-60.

15. Morton, B.R., "Modeling Fire Plumes", 10th Symposium (International) on Combustion, The Combustion Institute, 1965, pp. 973-982. 